


\title{
Centimeter
}

Association for Information and Image Management

1100 Wayne Avenue, Suite 1100

Silver Spring. Maryland 20910

301/587-8202

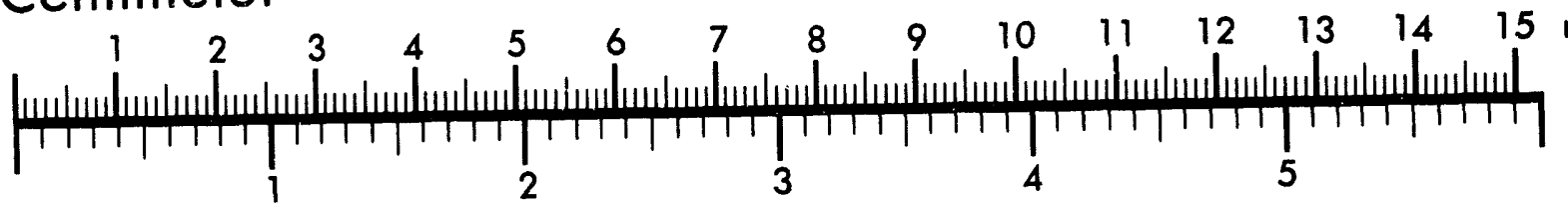

Inches
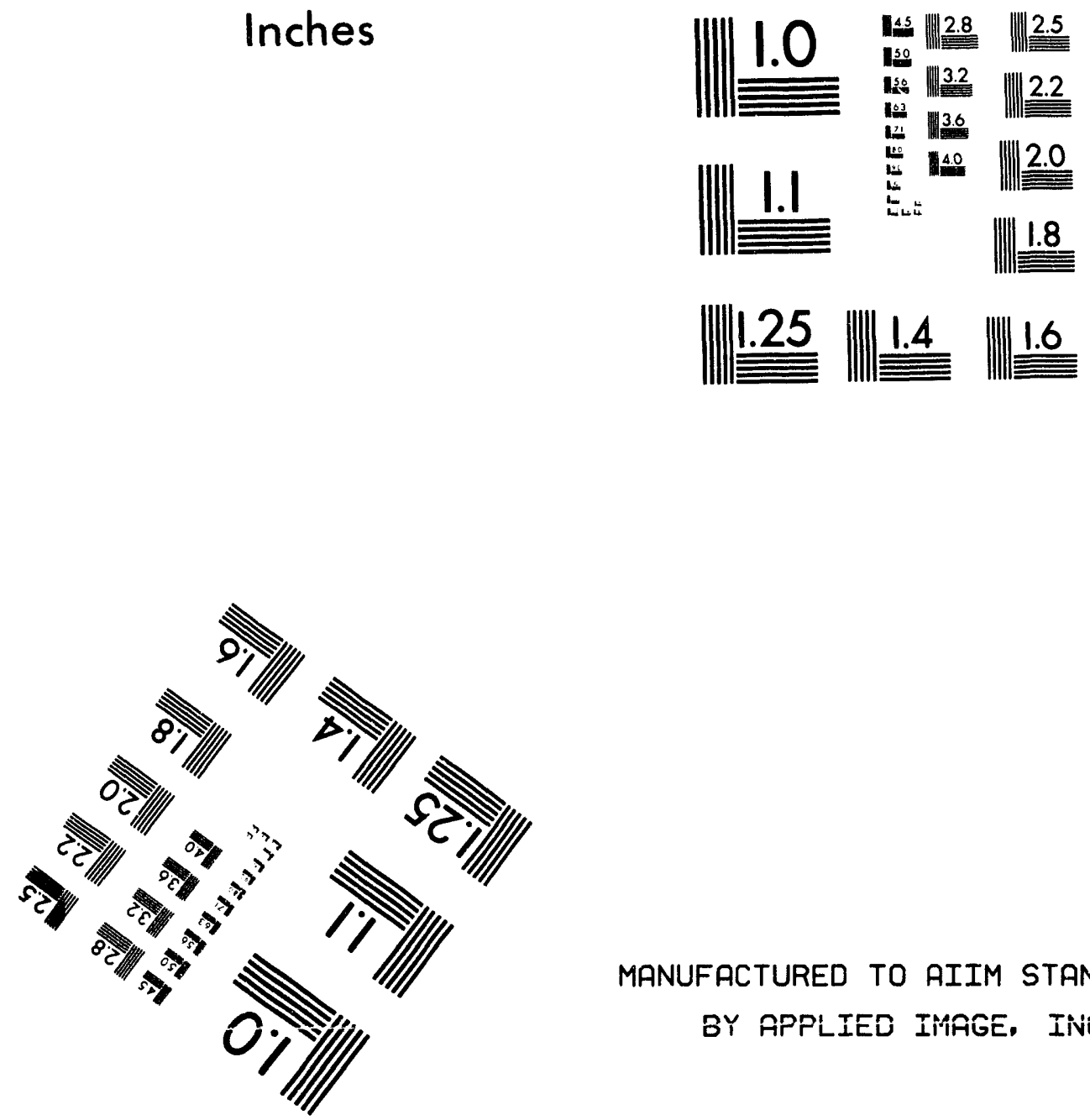

MANUFACTURED TO AIIM STANDARDS

BY APPLIED IHAGE, INVC.

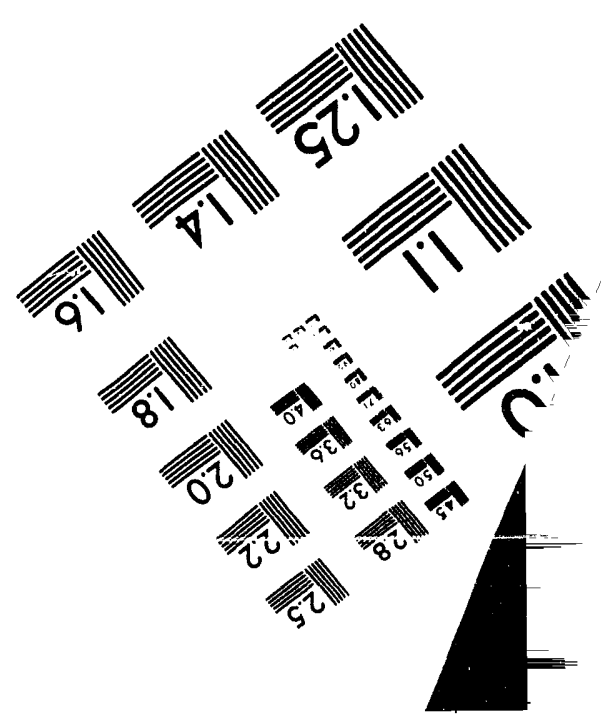



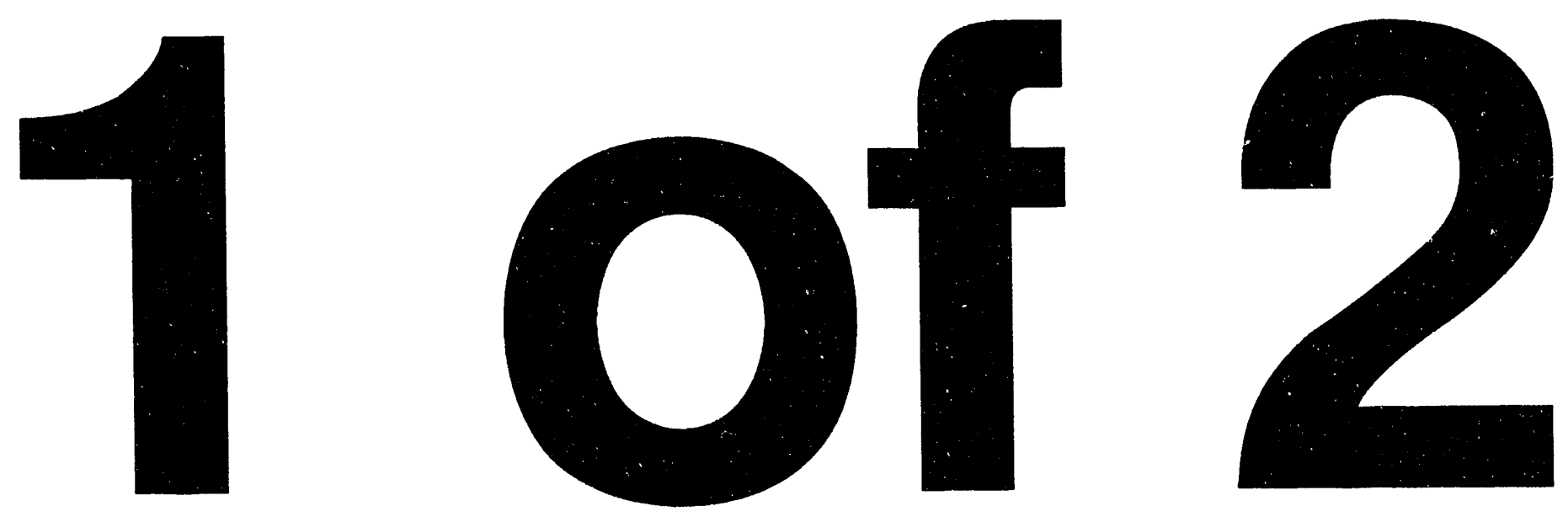
DOE/ER/40702-4

TUFTS UNIVERSITY

Tufts University

July 15, 1993

\section{HIGH ENERGY PHYSICS}

AT

TUFTS UNIVERSITY

U. S. DEPARTMENT OF ENERGY

GRANT

DE-FG02-92ER40702

PROGRESS REPORT

$1992-1993$

RECENT

AUG 041993

OSTI

NOTICE

This report was prepared as an account of work sponsored by the United States Government. Neither the United States nor the Department of Energy, nor any of their employees nor any of their contractors, subcontractors, or their employees, makes any warranty, express or implied, or assumes any legal their employees, makes any warranty, express or implied, or assumentility for the accuracy, completeness, or usefulness of any information, apparatus, product or process disclosed or represents that its use would not infringe privately owned rights. 


\begin{abstract}
The Tufts High Energy Physics Group here presents its report for the period June 15, 1992 to June 15, 1993 describing our progress and achievements since our prior report, DOE/ER/40702-2 [July 15, 1992]. Research during this period was supported under Grant DE-FG02-92ER40702.
\end{abstract}

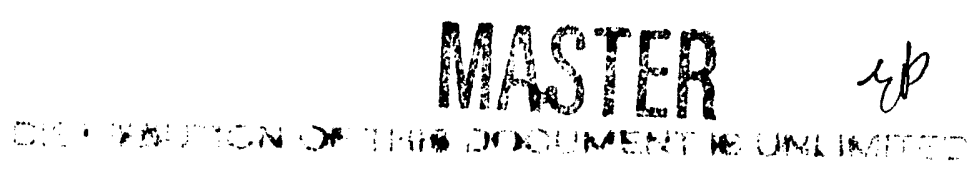




\section{CONTENTS}

\section{Introduction}

1. Overview

2. Personnel 7

\section{Experimental projects}

3. Fermilab fixed target experiments

A. Neutrino interactions in the 15-foot Bubble Chamber 9

B. Study of charm and charm-strange states in the Fermilab TPS 11

C. Study of charm and beauty states in the Fermilab TPS 21

D. Neutrino oscillations at the Fermilab Main Injector 26

4. Soudan II nucleon decay project

5. Physics at the proton-antiproton collider at $\sqrt{s}=1.8 \mathrm{TeV} \quad 61$

6. The Solenoidal Detector for the Supercollider 70

$\begin{array}{ll}\text { 7. Neutrino telescope proposal } & 78\end{array}$

Theoretical projects

8. Polarization in massive quark and hadron production $\quad 79$

$\begin{array}{ll}\text { 9. Production characteristics of top quarks } & 81\end{array}$

10. Scattering, spin dependence and mass corrections in Skyrmion models 84

\section{Facilities}

11. Computation and networking

\section{Communications}

12. Communications $1992-1993$
A. Experimental
B. Theoretical 


\section{INTRODUCTION}

\section{Overview}

The Tufts High Energy Physics Group here reports its research activity and accomplishments for the year preceding July 15, 1993. Much of our work is on the continuing analysis of data from our three primary research projects: (1) The Fermilab fixed-target study of heavy flavor production, E769 with 400 million events, is now yielding Physical Review Letters publications at a gratifying rate, and the analysis of its successor, E791 with an immense pool of 20 billion recorded events, is at the point where its full potential power is becoming evident. (2) The Soudan-II project is producing a steady stream of data from a detector that is now $90 \%$ of its ultimate size, and with its veto-shield array - Tufts' particular responsibility - covering more than $95 \%$ of the total $4 \pi$ solid angle. Data collection and analyses searching for nucleon decay candidates, cosmic muon sources, cosmic-ray neutrino oscillation phenomena and monopoles proceed routinely. Journal publications as well as conference reports are now appearing regularly, and $\mathrm{PhD}$ theses are being completed, including Tufts' second Soudan-II thesis which was finished during June 1993. A novel, and possibly prescient, byproduct of this work is a published suggestion by three Tufts scientists suggesting that the long-sought nucleon decay may already have been observed in the form of an excess of apparent cosmic-ray $\nu_{e}$-events in nucleon-decay detectors. (3) The Fermilab Tevatron collider program - CDF-is yielding a unique series of results at a great pace. Tufts' CDF researchers continue to work on CDF analyses - and especially on the timely pursuit of the top quark - even while their energies are stretched by their participation in the on-going 1992-93 CDF run. This run is yielding a new and much larger data pool from a detector enhanced by a new vertex detector and other improvements. In summary, our two Fermilab projects and our Soudan-II program are each well-immersed in their respective extended productive phases of analysis and publication, both of anticipated results and - we may hope - of unexpected revelations. These core programs collectively form a firm basis for our high energy group's research through much of the present decade.

A summary of our high energy physics program must include the work of our particle theorists - Prof. Goldstein and his students - who have continued their investigations during the past year. We note, particularly, the appearance in The Physical Review of the important paper, in collaboration with our CDF experimenter Prof. Sliwa and with Prof. R. Dalitz of Oxford, concerning their ingenious approach to the possible detection of the elusive top quark. Although still seemingly just a little beyond reach, "top" appears to be coming within range; tensions are mounting at the track, and it will not be surprising to many were the end of the race to occur within the year. (But "it ain't over 'til it's over," as Yogi Berra would remind us!)

A distinct feature of modern high energy research is the enormous size and cost of the experimental instruments required - both accelerators and detectors - and the consequent necessity for years of planning and hard labor to design and build these systems prior to their exploitation. To be daunted by this prospect would be to ignore a responsibility for the future of fundamental physics research and would neglect the futures of our younger 
researchers and students who must have the opportunity to mature and come to outshine their predecessors in the search for revelations about the nature of our universe. We have chosen to tackle couple of these "futuristic" projects and proposals, and are finding them to be both challenging in content and rewarding in the physical insights they provide along the way as we seek to invent new (and economical!) tools to probe the physical frontier.

Thus we have continued our work on several proposed projects which we anticipate will develop into exciting experiments before the end of this century. These proposals have been described in detail in our 1992 Report (DOE/ER/40702-2), and our work on several of them has progressed significantly. In particular, our physicists and technicians, working closely with their colleagues in the "Boston Muon Consortium" to prepare the barrel muon subsystem for the SDC detector at the SSC, are well along in their establishment of a factory unit at Tufts to manufacture the precision drift tubes for assembly into a prototype "supermodule" for SDC. Numerous hardware shop fixtures have been built to accomplish this. Also, a variety of simulation studies have been made to analyze the manufacturing tolerances which must be specified to achieve the required measurement precision for the SDC.

We have also continued to pursue development of our collaborative proposals to Fermilab to conduct short-baseline (P803) and long-baseline (P822) neutrino oscillation experiments based upon Fermilab's new Main Injector, now in preparation. For the former experiment, and in collaboration with Rutgers and Technion physicists, we have embarked on a novel investigation into the use of scintillating glass - rather than scintillating plastic - fibers to facilitate location of rare neutrino interactions in the nuclear emulsions of P803. For the latter experiment, P822, we are working to devise an economical yet massive and efficient neutrino detector to augment the present Soudan-II detector as a target for Fermilab-generated neutrinos. We are also working with the Fermilab "NUMI" project staff in the design of a beam for these experiments.

These "futuristic" studies and proposals share with high energy physics more generally the numerous uncertainties concerning the future level of support of fundamental science in this country, and elsewhere. We continue to devote a significant fraction of our research effort to them because they represent the most exciting scientific opportunities we can find and also because they are a good match to our capabilities and interests. We remain convinced that the chance for important successes based on such long-term planning and thoughtful commitment to the future still outweighs the risk of frustration arising from factors beyond our scientific control. We hope we will not be disappointed.

We close this overview with a brief resume of our specific progress in the several projects mentioned above. The individual items represent abstracts of the detailed descriptions to be found in subsequent sections of this Report. The items follow:

\section{Fermilab fixed-target experiments: hadroproduction}

Analysis of Fermilab experiment E769 on the hadroproduction of charm and charmstrange states continued, with participation by graduate students J. Astorga and D. Passmore and Profs. Napier and Milburn. Mr. Astorga is studying the strange cascade baryon signal with the hope of detecting charmed cascades and perhaps other charm baryons. Mr. Passmore is investigating channels producing $K_{s}$ mesons decaying well-downstream, 
as well as near, the segmented $\mathrm{Be}-\mathrm{Al}-\mathrm{Cu}-\mathrm{W}$ target, with a particular interest in $\mathrm{D}$-meson to $K_{s}$ decay signals. A major effort during the past year was to extract the downstream $K_{s}$ and $\Lambda$ events from the new data summary tapes resulting from the "re-reconstruction" (completed 3/92) of most of the raw data set to achieve an improved fitting of charm decay vertices. This was done to increase the overall yield as well as to reduce the background substantially. Two publications resulting from E769 work have appeared during the past year in Physical Review Letters and more publications are in preparation.

The successor experiment to E769, Fermilab E791, had data-taking runs at the Tevatron which were finished in early January 1992 with slightly over 20 billion event candidates being successfully logged on $(22,000) 8 \mathrm{~mm}$ video cassette tapes. The primary work on the subsequent tracking analysis of the raw data - an immense brute-force project occupying computer farms at Fermilab, Ohio State University and the University of Mississippi has been conducted by our colleagues elsewhere. During this period Prof. Napier has continued with his development of the event display system which will become useful as we begin to confront extracted physics signals. Prof. Milburn continued his investigation into the structure and calibration data for the spectrometer's magnetic field. We continue to expect this experiment to yield in excess of 100,000 charm decays after reconstruction as well as evidence of B-meson hadroproduction.

\section{Fermilab fixed-target experiments: neutrino studies}

The analysis of the 15-foot Bubble Chamber exposure of a Neon-Hydrogen mixture to the Tevatron neutrino beam (E632) was effectively completed by Mr. Willocq who, working with Profs. Kafka and Schneps, finished his dissertation on coherent interactions in $M_{\text {ay, }} 1992$, and saw through to publication an article on the subject in The Physical Review. While this represents the completion of Tufts' immediate research supporting the E632 program, the Collaboration is straining to increase the size of the unbiased event sample, hopefully through the continued participation of our Russian colleagues. The current economic situation in the former Soviet Union makes this prospect uncertain. The Tufts group expects to maintain contact with the Collaboration in the event that a sufficiently enhanced data pool eventually emerges.

\section{Soudan II nucleon decay project}

During the period of this Report, Soudan-II collected data routinely with an increasing fraction of the system in operation - about $90 \%$, at present - and with routine analysis and scanning of a variety of interesting signals. These include candidates for nucleon decay events, accompanied by their low-energy neutrino-interaction backgrounds, and candidate neutrino interactions to witness possible neutrino oscillation phenomena - or an exotic nucleon-decay channel! Analyses of data for other processes included a monopole search, and studies of cosmic-ray muons continued. At Tufts, the principal analytical effort was concentrated upon studies of multiple-muon processes (Messrs. Sundaralingam and Ewen) and on partially-contained events (Mr. Leeson), under the direction of Profs. Mann and Oliver. Mr. Sundaralingam's work culminated in his Ph.D. thesis, on the heavy-light ratio in primary cosmic radiation, which he successfully defended in early June, 1993. The phenomenological study in which Profs. Mann and Kafka, with Mr. Leeson, propose a nucleon-decay rather than an oscillation interpretation for the anomalously small atmo- 
spheric $\nu_{\mu} / \nu_{e}$ ratio, was published last year and has generated a gratifying, if controversial, response from the cosmic-ray neutrino community. These analyses continue, with a particular emphasis on the mounting accumulation of Soudan-II data on the matter. The Tufts group continued to participate at a modest level in the installation of the now essentially complete primary veto shield system at the mine. However, the enhancement of this system by the addition of refurbished HPW proportional-counter modules, and further by recycling to it the large set of similar modules from TASSO, has had to be deferred for lack of funds. Members of the Soudan Group again spent, as during the previous year, a significant effort in connection with the long-baseline neutrino oscillation proposal to Fermilab, P822.

\section{Physics at the Tevatron Collider (CDF)}

Prof. Sliwa (DOE Outstanding Junior Investigator), his students Mr. Benjamin and Ms. Bellino, and his post-doctoral Research Associate Dr. Timko, continued to work on the analysis of past CDF data and on participating in the execution of the current 199293 CDF data-collection run. In particular, Dr. Timko has the important responsibility, within CDF, for maintaining and testing the LEVEL3 trigger algorithms. Prof. Sliwa's analytical studies continue to concentrate on refining and applying a new and powerful technique for the detection of top quarks in the "lepton +4 jet" final state. The method, a modification to the original and elegant procedure developed by Profs. Goldstein and Dalitz (of Oxford) for analyzing top-quark events in the "di-lepton +2 jet" final state, was developed jointly by Profs. Goldstein, Sliwa and Dalitz and has been published in The Physical Review D. Prof. Sliwa's students, Ms. Bellino and Mr. Benjamin, have completed their analyses of $Z_{\gamma}$ and $W \gamma$ processes, respectively, and are writing up their results for their dissertations. A new graduate student, Mr. K.Karr, joined Prof. Sliwa's CDF group this summer.

\section{Solenoidal Detector for the SSC (SDC)}

Profs. Mann and Milburn continued to work intimately with Harvard and Brandeis colleagues in the "Boston Muon Consortium" in preparing to build the very large barrel muon subsystem for the SDC. This work is in collaboration with several other university laboratories arround the country, and with workers at the SSC laboratory in Texas. Tufts' contributions include the detailed design and preparation of tooling and of manufacturing stations for making the precision drift-tube modules needed to assemble at SSC a large prototype "supermodule" for the huge SDC detector. A special "clean room" has been installed at Tufts for the assembly of our share of the up to 9-meter long units. On another front, a series of computer simulations of the electrical and mechanical characteristics of the drift-tube structure have been carried out to establish the dependence of tube performance and precision upon variations in operating conditions and upon the extent to which manufacturing tolerances are specified and kept. The results have been published in three SDC Notes. Our primary goal during the past year has been to commence actual manufacture of prototype SDC drift-tube modules at Tufts before the end of summer, 1993. This remains a viable hope at this writing. 


\section{Neutrino oscillations at the Fermilab Main Injector (P803-P822)}

Work has continued on these two proposals, P803 for a short-baseline study based upon a large emulsion stack in an electronic spectrometer on the Fermilab site and P822 for a long-baseline experiment using a calorimeter $735 \mathrm{~km}$ distant in the Soudan-II underground laboratory. Much of the development during the past year has been based at Fermilab where the "NUMI" group has mounted a detailed effort toward refining the engineering design and evaluating the potential costs were Fermilab to proceed with various neutrino oscillation proposals. Certain specific studies have been mounted at Tufts during the period, in conjunction with NUMI and with the P803 and P822 collaborations. These include the initiation, by Profs. Schneps and Oliver, of a study in collaboration with Rutgers and Technion scientists to develop a new kind of glass scintillating fiber for use with the emulsion stack in P803. These would be assembled into an ultra-fine-grain hodoscope to enable the electronic preselection of neutrino-interaction candidates which will greatly simplify the task of microscopic emulsion scanning - and which is an absolute essential for this experiment. This work has proceeded to the point where samples of the new glass "preforms"(cylinders of $1 \mathrm{~cm}$ diameter not yet drawn into fibers) doped with rare-earth elements have been made by a novel vapor-deposition technique (at Rutgers) and are being evaluated at Tufts by Prof. Oliver as particle detectors.

For P822, Prof. Oliver is developing and testing a simplified, and relatively inexpensive, proportional tube structure which might serve as the active component of a multikiloton detector of Fermilab neutrinos at the Soudan mine.

Dr. Willocq, our recent Ph.D. from the E632 program, spent much of the past year carrying out Monte Carlo studies to refine our understanding of the problem of resolving $\nu_{\tau}$ interactions efficiently using the P803 spectrometer system; this knowledge is vital to the prediction of the running time and scanning effort involved.

A new proposal to Fermilab, P872, has been submitted. This experiment would use emulsions plus the P803 detector in a "beam dump" experiment at the Tevatron. The object would be to find $\nu_{\tau}$ interactions in the emulsion. The $\nu_{\tau}$ would come from $\mathrm{D}_{s}$ decays, the $\mathrm{D}_{s}$ being produced by $800 \mathrm{GeV}$ protons in the dump. P872 would not only be a trial of the P803 apparatus, but could "discover" tau-neutrinos.

\section{Theoretical projects}

Prof. Goldstein during this period has continued his development of kinematic methods for identifying top-quark production from $p \bar{p}$ collisions and for determining the mass of this elusive elementary particle. His recent progress, in collaboration with Prof. R. Dalitz of Oxford, embraces the inclusion of density matrices in their geometrical method, and the making of simple simulation studies that incorporate spin effects. Prof. Goldstein also continued an investigation into polarization in massive quark and hadron production and QCD subprocesses, this in collaboration with his former student, Dr. W.G.D. Dharmaratna who is now at Florida State University. These studies represent a natural generalization of Dr. Dharmaratna's dissertation work and suggest certain direct experimental tests of perturbative QCD. Finally, Prof. Goldstein reports progress in his work with his student, Mr. L. Gamberg, on extended Skyrmion models. 


\section{Computing and networking}

Our program to upgrade the high energy physics computing facilities continued at a modest rate this past year with the conversion of another VAXstation-3100 to a 35 MIPS VAXstation- 4000 and with the acquisition of additional disk units to contain the working files from our burgeoning analysis efforts. As we become involved with an everincreasing flow of data from Soudan-II and our Fermilab experiments, we are being forced to view our $8 \mathrm{~mm}$ tape-drive units, and to a lesser extent our video terminal equipment, as akin to "consumable supplies." Being mechanical in nature, the tape drives routinely wear with use and begin to fail; so we need to develop a pool of spares and a service "pipeline" (to Fermilab, at the moment) to keep our operations going efficiently. Terminal failures, on the other hand, are essentially unpredictable and seem to reflect variations in intrinsic durability under near-continuous operation; replacement, rather than repair, has proven more economical in most cases. These increasing maintenance problems are surely well-known at larger laboratories, but they also witness the growing level of dependence of Tufts' high energy physics research program upon our highly stressed computing resources. 


\section{Personnel}

The following sections list the personnel who have participated in this grant during the year reported:

\section{A. Project Directors/Principal Investigators}

Professor W. A. Mann

Professor R. H. Milburn

Professor J. Schneps

As co-Directors, they have been responsible for administration of project affairs, with considerable vital help from their colleagues.

\section{B. Tenured Faculty}

Professor G. R. Goldstein

Professor W. A. Mann

Professor R. H. Milburn

Assoc. Prof. A. Napier

Assoc. Prof. W. P. Oliver

Professor J. Schneps

\section{C. Non-tenured (tenure-track) Faculty}

Asst. Prof. K. J. Sliwa (1989-present)

(DOE Outstanding Junior Investigator, from 1990)

\section{D. Research Faculty}

Research Professor T. Kafka

\section{E. Postdoctoral Research Associates}

Dr. Mark Timko

(working at Fermilab with Prof. Sliwa on the CDF project.)

Dr. Stephane Willocq (9/1/92-3/31/93)

(Dr. Willocq was supported, in part, by Fermilab for work in connection with the NUMI project. On April 1, 1993, Dr. Willocq joined the Yale University group working at SLAC on SLD.)

\section{F. Graduate Students}

The number of graduate students supported on research assistantships has been six or seven annually (one in theory, the rest in experiment). In addition we had several students working with us who had other means of support, e.g. teaching assistantships. Here we list the students on board during the past year, indicating their status, means of support, and area of research.

J. Astorga* (TA \& RA-E791)

M. Roach-Bellino* (RA-CDF)

D. Benjamin* (RA-CDF)

J. Carozza* (P-E545)

B. Ewen* (TA \& RA-Soudan) 
L. Gamberg* (RA \&P-Theory)

W. Leeson* (TA-Soudan)

D. Passmore* (RA-E769)

N. Sundaralingam* (RA-Soudan) Ph.D. thesis completed, June 1992.

An asterisk indicates all requirements for the Ph.D. are completed except for the thesis. $\mathrm{RA}=$ research assistantship, $\mathrm{TA}=$ teaching assistantship and $\mathrm{P}=$ private means.

\section{G. Graduate Technical Assistants}

In addition to those named above, a number of graduate students have been employed for special projects during the summers of 1992 and 1993. Certain of them, so indicated, are supported from the Texas National Research Laboratory Commission (TNRLC) grant we share with other members of the "Boston Muon Consortium" to work on SDC-related activities. Other support includes DOE-Grant funds, summer teaching appointments, and special faculty funds.

T. Bernard (1993-E791)

K. Karr (1992-SDC/TNRLC, 1993-CDF)

M. Pfenning (1992-SDC/TNRLC, 1993-SDC/TNRLC \& Soudan)

D. Wall (1993-SDC/TNRLC)

G. Yasuda (1992-SDC/TNRLC)

\section{H. Summer Undergraduate Student}

A. Kosmopoulos, Tufts Class of 1995.

Mr. Kosmopoulos is working on the Soudan-2 Project as a volunteer during the summer of 1993. A Junior, he is majoring in Physics and looks forward to graduate school and a professional career.

\section{I. Supporting Staff}

L. McMaster, Shop supervisor and building manager

D. Dupuis, Machinist

D. DeStefano, Secretary-Bookkeeper $(p / t)$

R. Fleischer, Secretary-Bookkeeper $(\mathrm{p} / \mathrm{t})$ 


\section{EXPERIMENTAL PROJECTS}

\section{A. Neutrino interactions in the 15-foot Bubble Chamber}

\section{A.1. Neutrino interactions in Neon}

(Fermilab E-632: Profs. Kafka, Mann, Milburn, Napier, and Schneps, and Dr. Willocq, with collaborators from Birmingham, Brussels, CERN, Chandigarh, IHEP Serpukhov, Imperial College London, ITEP Moscow, Jammu, Moscow State University, Munich, Oxford, Rutherford Appleton Laboratory, Saclay, Berkeley, Fermilab, Hawaii, I.I.T., and Rutgers)

The Europe-India-USA-Russia collaboration collected its data in two Tevatron runs in 1985 and 1987. Information about the data taking runs and about the activities of the Tufts Film Analysis Facility until its closing in March 1990 was given in our 1986-1991 report $^{1}$ (p. 12), together with a description of the early physics analysis and results. Data from the 1985 run yielded the first published papers, including measurements of dimuon production and the neutral current cross-section.

First results from the analysis of the combined 1985 and 1987 data, based on the low-multiplicity $\left(n_{c h}=2-4\right)$ data sample, were published this year. ${ }^{2}$ Ref. 2 summarizes results on coherent production of single piuns and $\rho^{0}$ 's in (anti)neutrino interactions with the neon nucleus, as obtained by Tufts graduate student Stephane Willocy, and marks the end of the era of intense Tufts involvement in E632. As detailed in Dr. Willocq's thesis ${ }^{3}$ and in our 1992 Progress Report ${ }^{4}$ (p. 8), this is an investigation uniquely suited for the bubble chamber technique capable of detecting slow proton and nuclear fragment tracks. The phase space region explored is characterized by low $-Q^{2}$ (momentum transfer) and large $\nu$ (energy transfer), distinctly separate from the more commonly investigated deep inelastic scattering where the perturbative theory of strong interactions is appropriate. The results for the cross-sections for coherent $\pi$ and $\rho$ production were aptly confronted with predictions of theory incorporating the PCAC hypothesis and the idea of hadron dominance of weak interactions showing that the validity of these ideas extended into the Tevatron energy regime.

The prospects for the E632 future are uncertain. The collaboration obtained about 1000 charged-current events from the 1985 run, and, at first, only 30,000 frames (about $10 \%$ ) of the 1987 film were completely measured yielding $2000 \mathrm{CC}$ events. All of the US and European institutions then ceased operating their scan shops. Expecting this outcorne, the collaboration accepted three Russian institutions hoping that they would continue processing the E632 film. So far, the amount of data contributed by the Russians has only doubled the meagre statistics, and the difficult economic situation in Russia may mean that they will not be able to continue without external help.

However, the collaboration is actively pursuing the physics analysis with whatever resources are available:

- 1 production: a paper is being prepared for submission.

- Slow protons and rescattering within the target neon nucleus: Graduate student Mary Lauko of Rutgers has completed her Ph.D. thesis and a paper summarizing her work will follow. 
- General features of hadron production: multiplicity distributions, inclusive distributions, energy flow, rapidity correlations.

- $D_{s}$ and $D_{s}^{*}$ : A search for $\mathrm{D}_{s}$ has been performed and the presence of a signal in the raw data after preliminary cuts has been established.

- Formaition length: The analysis is under way to investigate the length within which the nascent outgoing hadronic system has a reduced probability of interaction within the rget nucleus.

- Resonance production: $\rho^{0}(770), \mathrm{K}^{* \pm}(890)$, and $\Sigma^{ \pm}(1385)$.

\section{A.2. Neutrino interactions in deuterium}

(Fermilab E-545: Profs. Kafka, Mann, Napier, and Schneps, and Mr. J. Carozza, with collaborators from I.I.T., Maryland, and Tohoku (Japan).)

We continue to analyze data from a 15-Foot B.C. exposure obtained in 1978, using a final $\nu$ D DST prepared in 1982. Mr. J. Carozza i presently writing up results of his investigations of the production of vector mesons anc strange particles, and their role in u-quark fragmentation.

\section{Section 3.A References}

1. High Energy Physics at Tufts University, DOE/ER/40085-23 (1991).

2. S. Willocq et al., Phys. Rev. D47, 2661 (1993).

3. S. Willocq, Coherent Production of Pions and Rho Mesons in Neutrino Charged Current Interactions on Neon Nuclei at the Fermilab Tevatron (Ph.D.Thesis), Tufts University, April 30, 1992, DOE/ER/40702-1.

4. High Energy Physics at Tufts University, DOE/ER/40702-2 (1992). 


\section{B. Study of charm and charm-strange states in the Fermilab TPS}

(Fermilab E769 - Profs. Napier and Milburn; Graduate Research Assistant D. Passmore; Graduate Students J. Astorga and T. Bernard; with collaborators from C.B.P.F./Brazil, U. Mississippi, Fermilab, Northeastern U., U. Toronto, U. Wisconsin, Yale U.)

E769 is a study of charm hadroproduction which used $250 \mathrm{GeV}$ beams of $\pi^{+}, \pi^{-}, K^{+}$, $K^{-}$, and protons on four different types of foil targets - beryllium, aluminum, copper, and tungsten. The experiment was conducted in the Tagged Photon Spectrometer (TPS) at Fermilab. More than 400 million events were written to tape by the end of data-taking in 1988. Reconstruction of the data is complete, and about 4000 charm particle decays are available for analysis. Two papers have recently been published in Physical Review Letters: 'Feynman-x and Transverse Momentum Dependence of $D^{ \pm}$and $D^{0}, \bar{D}^{0}$ Production in 250 $\mathrm{GeV} \pi^{-}$-Nucleon Interactions,' in Phys. Rev. Lett. 69 (1992), p. 3147, and 'Atomic Mass Dependence of $D^{ \pm}$and $D^{0}, \bar{D}^{0}$ Production in $250 \mathrm{GeV} \pi^{ \pm}$-Nucleon Interactions' in Phys. Rev. Lett. 70 (1993), p. 722. A third paper entitled ' $D^{* \pm}$ Production in $250 \mathrm{GeV} \pi^{ \pm}$ N Interactions' was submitted to Physical Review Letters in April 1993, and it appeared as Fermilab preprint FERMILAB-Fub-93/081-E. A fourth paper titled 'Absolute Cross Sections and Incident Particle Dependence of Charm Meson Hadroproduction at $250 \mathrm{GeV}$ ' is undergoing final revisions, and will likely be submitted this summer.

Three talks were presented on E769 data at the Fermilab meeting of the APS Division of Particles and Fielcis in November, 1992, by Chris Darling (Yale), Steve Takach (Yale) and Gilvan Alves (C.B.P.F) based on their Ph.D. thesis research. Chris Darling and Steve Takach have now completed their dissertations and have left Yale for post-doctoral positions. Ali Rafatian (U. Miss) presented a talk on $D^{* \pm}$ production at the April, 1993, APS meeting in Washington, D.C., and he is expected to complete work on his thesis this summer. At Tufts, David Passmore has nearly completed a final set of DST tapes containing $K_{s}$ and $\Lambda$ decays and Juan Astorga is searching for charm baryon decays to $\Xi^{-}$and $\bar{\Xi}^{+}$. Tim Bernard joined our group last year and he is presently looking at charge asymmetries in Feynman-x distributions of $D^{ \pm}$mesons, a topic of considerable current interest.

The following sections describe forthcoming publications, progress in the study of $D$ decays to final states involving $K_{s}$ mesons, the search for $\Xi_{c}$ decays to $\Xi^{-}$final states, and other studies continuing at Tufts.

\section{B.1. Re-reconstruction of charm events}

As described last year, the re-reconstruction of all pair-strip data was carried out at Fermilab in order to correct an alignment error. It was expected that the new pair-strip tapes would have full error matrices included for tracks originating from decays downstream of the silicon microstrip detectors (SMDs). This would have allowed proper fits for many more of the neutral strange particle decays, as well as $\Xi^{-}$and $\Omega^{-}$baryon decays and charge conjugate (C.C.) states, which are being studied at Tufts by David Passmore and Juan Astorga. Unfortunately, due to an error in building the source code files, the code for the full error matrices was apparently not included in the executable files used to rereconstruct the data. The error banks exist on the tapes, but they do not contain correct 
information. It was decided to proceed with the existing tapes and give up the possibility of doing fits to secondary decays beyond the SMDs.

The total number of charm decays depends on the particular selection cuts used; however, about 4000 decays of D-mesons are found in the channels $D^{+} \rightarrow K^{-} \pi^{+} \pi^{+}$, $D^{0} \rightarrow K^{-} \pi^{+}$, and $D^{0} \rightarrow K^{-} \pi^{+} \pi^{+} \pi^{-}$and charge conjugate states. Other charm states observed include $D^{*+} \rightarrow D^{0} \pi^{+}, D_{s}^{+} \rightarrow K^{+} K^{-} \pi^{+}$, and $\Lambda_{c}^{+} \rightarrow p K^{-} \pi^{+}$(and C.C.).

\section{B.2. Study of $D^{* \pm}$ Mesons}

All of the $D^{* \pm}$ events from E769 have now been analyzed, and a paper has been submitted to Physical Review Letters based on Ph.D. theses of Jussara de Miranda (C.B.P.F., April 1992), Sandra Amato (C.B.P.F., May 1992), and Ali Rafatian (U.Miss., nearing completion). We have measured the differential cross sections with respect to Feynman$\mathrm{x}\left(x_{F}\right)$ and transverse momentum $\left(p_{T}\right)$ of the produced vector $D^{*}$ mesons. In the range $0.1<x_{F}<0.6$ we find the distribution is well fit by a form $\left(1-x_{F}\right)^{n}$ with $n=3.4 \pm 0.4 \pm 0.1$. Within errors, this value is consistent with the $n$ value found for the pseudoscalar $D$ mesons in our previous publication. We find that the difference between the values of $n$ for leading and non-leading $D^{*}$ 's is $1.7 \pm 0.8$, indicating a weak leading particle effect. The $d \sigma / d p_{t}{ }^{2}$ distribution was parametrized in the form $\exp \left(-b p_{T}^{2}\right)$ over the range $0<p_{T}^{2}<4 \mathrm{GeV}^{2}$ and the fit result was $b=0.67 \pm 0.08 \pm 0.04 \mathrm{GeV}^{-2}$. This is somewhat less than the values of $0.99 \pm 0.06$ and $1.08 \pm 0.08$ found for $D^{+}$and $D^{0}$ respectively (including C.C.). However, one expects a smaller $b$ value for higher mass states. The atomic number dependence was parameterized as $A^{\alpha}$ as in our previous study of the pseudoscalar $D$ mesons. We find a value of $\alpha=0.98 \pm 0.06 \pm 0.02$ consistent with the pseudoscalar results.

\section{B.3. $D$ Meson decays to $K_{s}^{0}$ final states (at Tufts)}

As reported last year, the decays of $D^{0}$ and $\overline{D^{0}}$ to $K_{s}^{0} \pi^{+} \pi^{-}$are being examined by David Passmore. Starting with the pair-strip data summary tapes, he selected only events containing one or more $K_{s}^{0}$ for further examination. He then studied the effects of various cuts which were already being used to isolate $D$ decays to charged kaon final states, as well as other cuts, with the aim of choosing the cuts so as to maximize the signal (actually the ratio of signal to total sample size).

His analysis is being repeated with the re-reconstructed data set. This should provide a much lower background to the $D$ signal, and also provide a cleaner $K_{s}^{0}$ sample as well. He spent the first third of this year in technical studies and development. The middle third was dedicated to upgrading the code to be compatible with the properties of the new data set. The rest of the year, continuing to the present, was spent stripping the data set using his new strip program.

The isolation of a sample of neutral kaons is oriented toward using the $K^{0}$ decay modes to study neutral $D$ charmed mesons. The isolation of a $\Lambda$ sample is a natural spinoff of this work since the topology of a $\Lambda$ decay is very similar to that of a $K^{0}$. A carefully made $\Lambda$ extraction will be useful for charmed baryon studies. A study of inclusive production of neutral strange particles may also be interesting, especially considering the special systematics of the E769 detector for looking at the $\dot{A}$-dependence of cross-sections. 
A new project was started in the last quarter to search for hyperon decay modes such as $\Lambda_{c}$ goes to $\Sigma \pi \pi$ and $\Xi_{c}$ goes to $\Xi_{g} \pi \pi$. A hyperon usually decays before reaching the magnets so its momentum is not measured. However the momentum can be inferred from the topology of the event, because the total momentum of the daughter particles has to point back to the production vertex. By assigning a momentum to the 'stub', analysis can proceed in the usual fashion. A 'stub strip' was run on half the data set and is currently being analyzed. Work is still in progress and no conclusive results have been obtained yet. This study involves charm baryon decay modes not being examined by other members of the E769 collaboration, and it may provide useful checks of the cross sections and/or limits resulting from Juan Astorga's study of cascade final states.

The upcoming year will be exciting as the results of these two studies come in. Hopefully the $D^{0} / K^{0}$ strips will yield a strong $D^{0}$ signal. The $D^{0}$ sample is not expected to be large enough to contribute to production studies but a look into the resonance structure of the decay of the $D^{0}$ into a $K^{0}$ and pions will be interesting. The prospects for a charmed baryon signal from the stub strip seem good. Events which pass both the stub strip and the $\Lambda$ strip will receive special attention since this subset should yield a clear signal of $\Xi_{c}$. Of primary interest will be the $\Lambda_{c}$ goes to $\Sigma \pi \pi$ sample which potentially has a large branching fraction but current world statistics are very poor. Figs. 3.B.3.1a,b show the $\Lambda$ and $K_{s}^{0}$ signals from the strip. About $74 \%$ of the strip is now complete.

\section{B.4. Search for $\Xi_{c}$ states (at Tufts)}

All the positive and negative beam data have been reprocessed from the improved data set. Cuts have been optimized for different momentum ranges of $\Xi_{s}$, and the yield has increased to approximately 8000 events.

Of the cuts studied, the ratio of impact parameter of the $\Xi_{s}$ (to the primary vertex) with its flight distance seems to be of special interest due to the clear separation between signal and background. Figs. 3.B.4.1a,b show the distribution of signal and background for this cut. We can see from these plots that most of the signal is distributed so that the ratio is less than 0.01 ; whereas approximately half of the background is located above this value. Figs. 3.B.4.2a, $\mathrm{b}$ show the significance and efficiency of the cut. The shaded areas on Figs. 3.B.4.3a,b show what is left and kept by doing this cut. It is expected that this cut will also greatly improve the signal to noise for charm baryons.

We have also searched for tracks which were reconstructed in the SMD, but were not linked with drift chamber (DC) tracks, and compared their production angles with those of $\Xi_{s}$ candidates. It is possible to diminish the background of $\Xi_{s}$ using this technique, and perhaps reduce the background for charm baryons as well. Fig. 3.B.4.4a shows the $\Xi_{s}$ signal and Fig. 3.B.4.4b is a subset obtained after requiring that the unlinked SMD track forms an angle less than $1.8 \mathrm{mrad}$ with a $\Xi_{s}$ reconstructed from DC tracks. Only about $20 \%$ of the $\Xi_{s}$ are associated with such tracks, and we are presently trying to learn why this percentage is not larger. 
With this clean sample of $\Xi_{s}$ we expect to search for charm baryons, such as

$$
\begin{gathered}
\Xi_{c}^{+} \rightarrow \Xi_{s}^{-} \pi^{+} \pi^{+} \\
\Xi_{c}^{+} \rightarrow \Lambda^{0} K^{-} \pi^{+} \pi^{+} \\
\Xi_{c}^{0} \rightarrow \Xi_{s}^{-} \pi^{+} \\
\Xi_{c}^{0} \rightarrow \Xi_{s}^{-} \pi^{-} \pi^{+} \pi^{+} \\
\Omega_{c}^{0} \rightarrow \Xi_{v}^{-} K^{-} \pi^{+} \pi^{+}
\end{gathered}
$$

As part of our charm baryon search for $\Xi_{c}^{0} \rightarrow \Xi_{s}^{-} \pi^{+}$we have found a clean $\Xi_{s}^{0}(1530)$ resonance signal. This sample can be found in Figure 3.B.4.5.

\section{B.5. Other studies at Tufts}

All of the topics require Monte Carlo calculations to tune analysis cuts, study reconstruction efficiencies, and estimate cross sections. We are currently running a Monte Carlo generator on the SGI Indigo workstation as well as on the two 4D/25 Datastation servers. Additional running at Fermilab may be necessary to generate a significant sample of Monte Carlo decays of charm baryons to be useful for comparison with our dataset.

\section{B.6. Summary}

After several years of preliminary results, E769 has reached the stage when final results are being published. Nine students have already received Ph.D. degrees based on E769 analysis, and four more, from Tufts, Mississippi, and Yale, are likely to finish in the next year. We look forward to final reports on $D_{s}^{+}, \Lambda_{c}^{+}$, and other charm baryons. We note, finally, that much of the analytical development we have described for E769 will carry over directly to the analysis of the 50-fold larger dataset for Fermilab E791, which is described in the next section. 

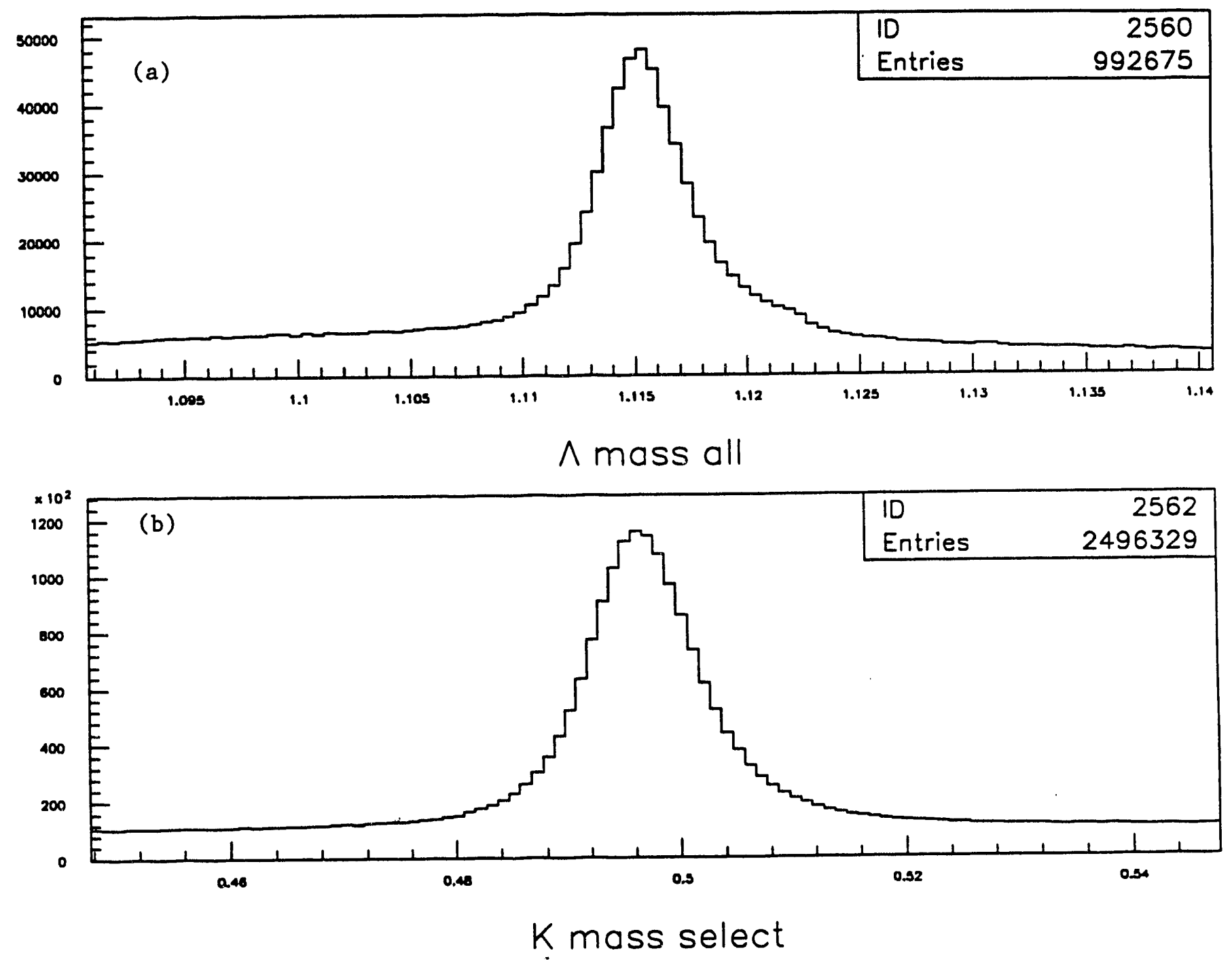

Figure 3.B.3.1 


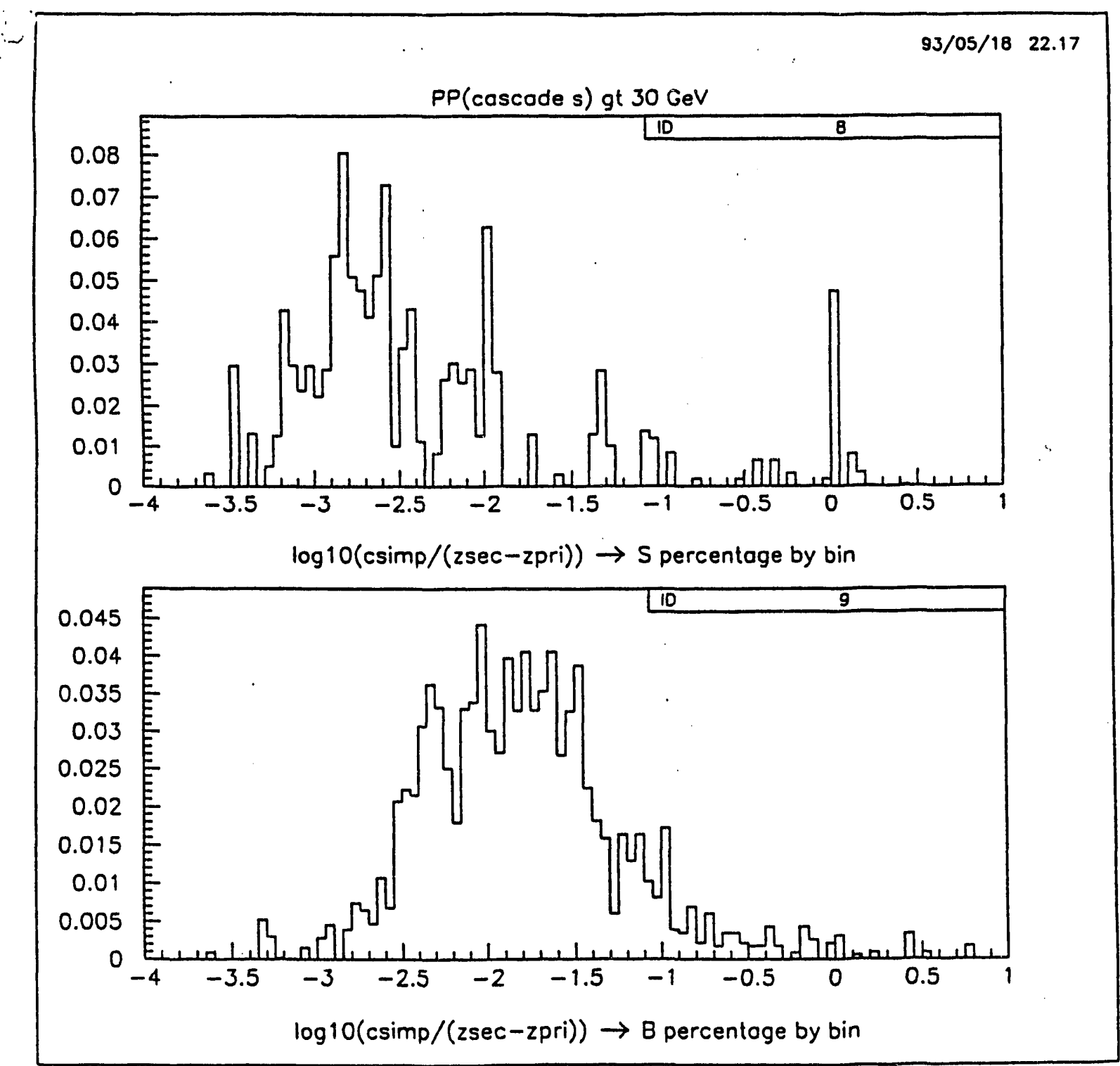

Figure 3.B.4.1 


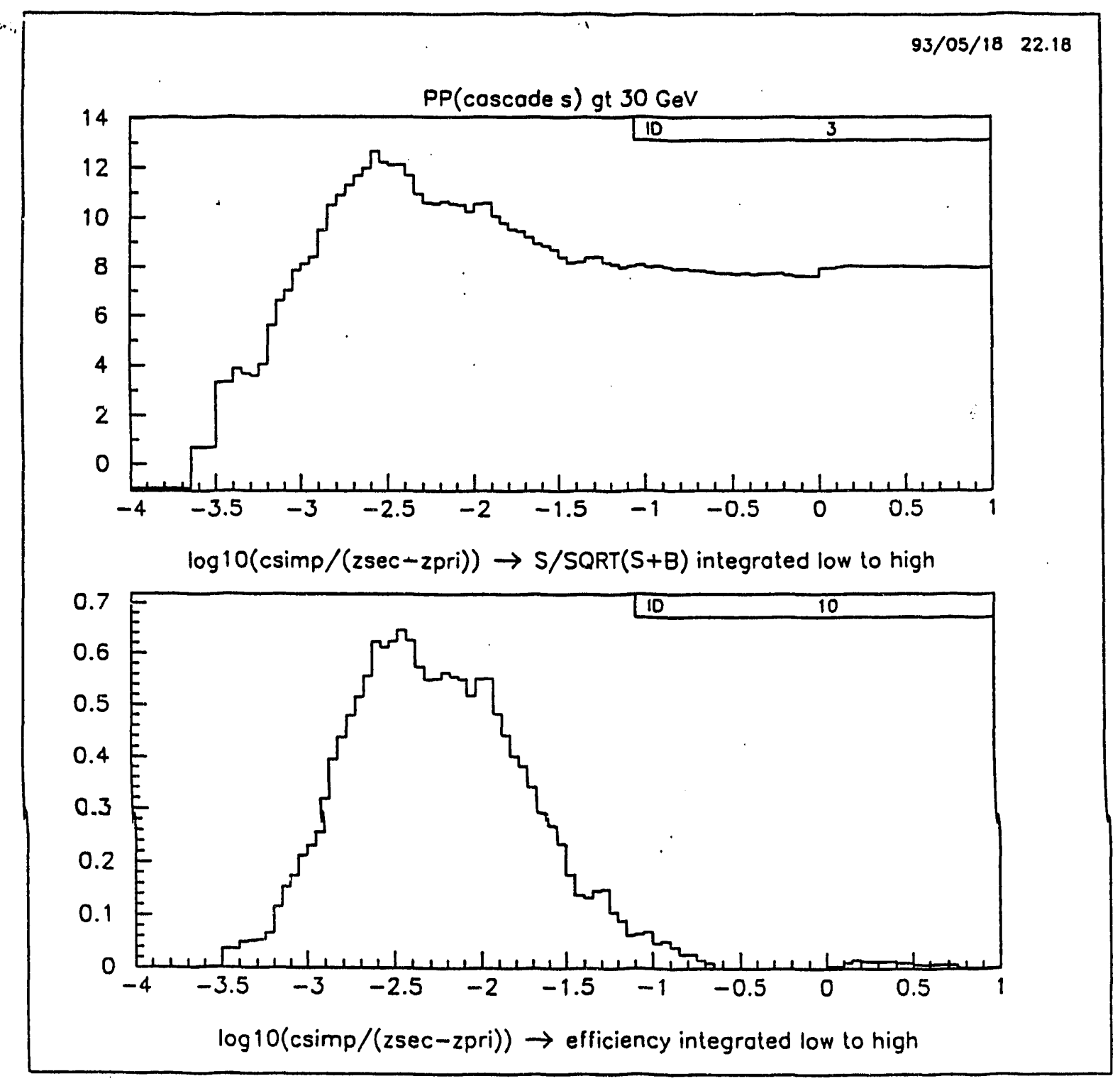

Figure 3.B.4.2 



Figure 3.B.4.3 



Figure 3.B.4.4 


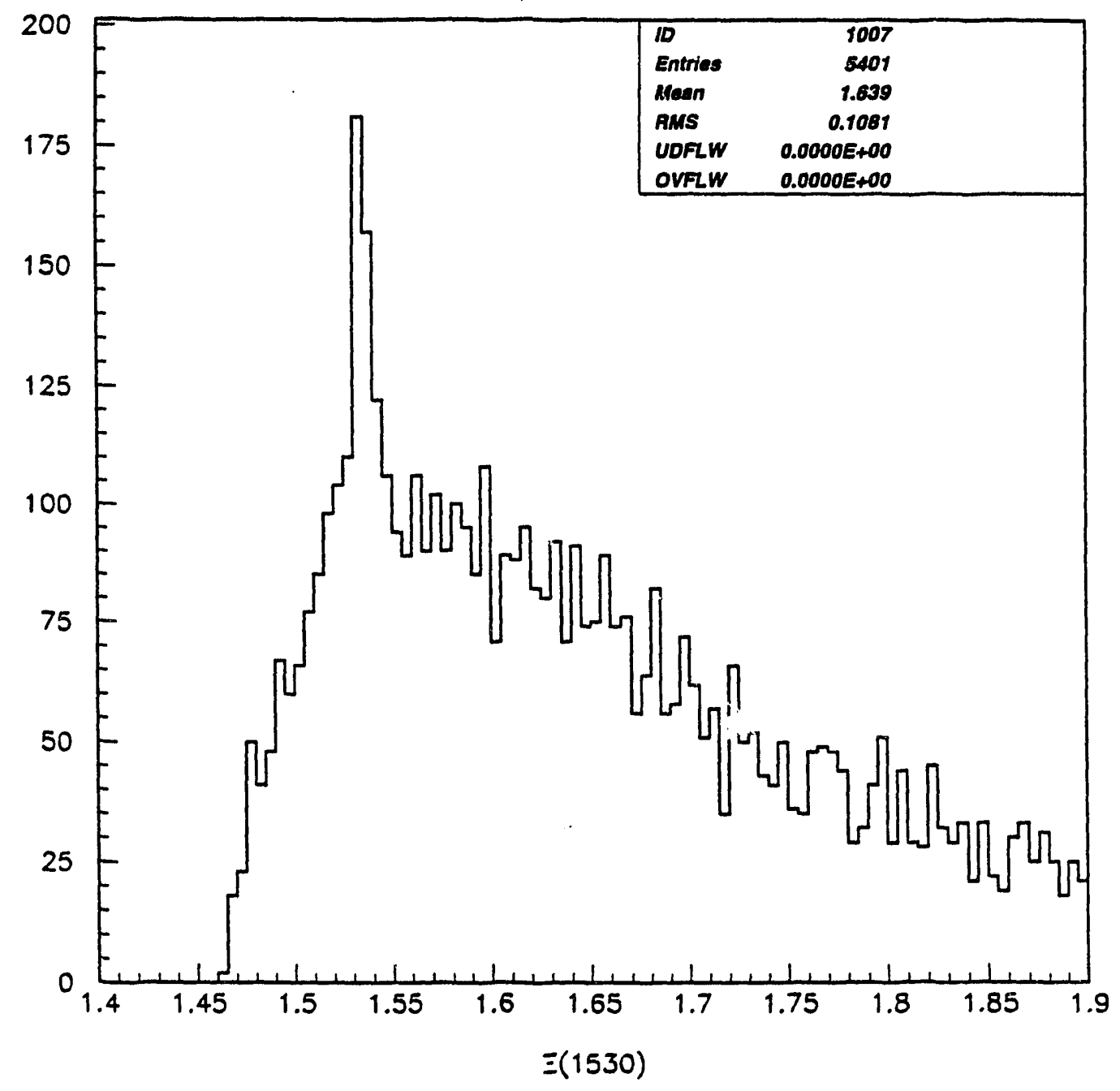

Figure 3.B.4.5 


\section{C. Study of charm and beauty states in the Fermilab TPS}

(Fermilab E791 - Profs. Milburn, Napier, Kafka, Schneps; Graduate student J. Astorga, with collaborators from C.B.P.F./Brazil, Fermilab, I.I.T., U. Mississippi, Ohio State U., Princeton U., U. Fed. do Rio de Janeiro, Tel Aviv U.,U.C.-Santa Cruz, U. of Cincinnati, U. of Wisconsin, Yale U.)

\section{C.1. Introduction}

The main goal of Fermilab E791 is to reconstruct 100,000 charm decays and perhaps several hundred beauty decays from a $500 \mathrm{GeV}$ beam of $\pi^{-}$and $K^{-}$incident on platinum and carbon targets. By the end of January, 1992, we had recorded 20 billion events on $24,0008 \mathrm{~mm}$ tapes. We expect this large data sample to allow detailed tests of current models of charm production and decay, provide more stringent limits on $D^{0}-\overline{D^{0}}$ mixing, allow precise lifetime measurements of charm baryon states, and search for leading particle effects at high Feynman-x. Results from reconstruction of about $2 \%$ of the data on farms of RISC computers at O.S.U., U. Miss., and Fermilab confirmed expectations. Figures 3.C.1.1 and 3.C.1.2 show some of the charm signals which were presented at the Fermilab Director's Review of E791 last November, and at the 1992 meeting of the Division of Particles and Fields at Fermilab. Figure 3.C.1.1 shows the results of a search for $D^{0}$ decays in the Cabibbo-favored mode $D^{0} \rightarrow K^{-} \pi^{+}$(upper left), as well as in the Cabibbosuppressed modes $D^{0} \rightarrow K^{-} K^{+}$(lower left) and $D^{0} \rightarrow \pi^{-} \pi^{+}$(lower right). Also shown is the $D^{*+}$ signal, evident in the mass difference histogram of $D^{*+}$ and $D^{+}$(upper right). Figure 3.C.1.2 shows histograms for $D^{+} \rightarrow K^{-} \pi^{+} \pi^{+}$(upper left), $D^{+} \rightarrow \pi^{-} \pi^{+} \pi^{+}$(upper right), $\Lambda_{c} \rightarrow p K^{-} \pi^{+}$(lower left), and $D^{0} \rightarrow K^{-} \pi^{-} \pi^{+} \pi^{+}$(lower right).

We now have nearly $20 \%$ of the data reconstructed and available for more detailed analysis. Two abstracts have been submitted to the Fifth International Symposium on Heavy Flavor Physics to be held in Montreal in July, 1993. These will present preliminary results from semi-leptonic decays and $\mathrm{D}$-meson production properties $\left(x_{F}\right.$ and $p_{T}$ differential cross-sections and $A$-dependence).

We estimate that the total yield of reconstructed charm decays will be greater that 200,000 , and will include about 2000 events with two fully reconstructed charm decays, based upon these early results. We note that this $2 \%$ data sample from E791 is already larger than the entire E769 dataset!

\section{C.2. Data Run}

The details of the data-taking were described in last year's progress report. We note that details of the data acquisition system have now been published in an article entitled: "The E791 parallel architecture data acquisition system" in Nuclear Instruments and Methods, A234 (1993), p. 535-542. The data acquisition system allowed events to be simultaneously written to tape on $428 \mathrm{~mm}$ Exabyte tape units at a total rate of $9.6 \mathrm{Mb} / \mathrm{sec}$. To avoid confusion, all 42 tape units were unloaded at the same time, when at least one of the tapes became $90 \%$ full $(2.1 \mathrm{~Gb}$ each). In our nomenclature, a "run" consists of the data contained on such a set of 42 tapes. It is occasionally easier to discuss data reconstruction and event filtering in terms of the number of runs processed. 


\section{C.3. Offline Analysis}

The immense size of the E791 dataset requires a huge number of processors for timely reconstruction. Ohio State University and the University of Mississippi are each running large "farms" of DECstations. Each computer "node" contains a $25 \mathrm{MHz}$ R3000 CPU from MIPS. To reconstruct $2 \times 10^{10}$ events will require about 400 node-years of these processors at $70 \%$ utilization. Farms of IBM and ACP-II processors are now working at Fermilab and CBPF in Rio de Janeiro. The to 1 CPU processing capacity of these four institutions may allow reconstruction of all E79 ata within about 3 years.

We are very near to the "release 6" version of the reconstruction software for E791. Release 5 has been operational since February 9, and a reasonably large amount of data has been processed with it. The longer interval preceding this new release may indicate that we are reaching a stable set of software with no major bugs and only minor revisions remaining. By now (late May) about 1800 million events have been reconstructed and stripped on the OSU farm, and about 700 million events each on the U. Miss. and FNAL farms, yielding a sample of about $3.2 \times 10^{9}$ events for study. In Rio de Janeiro, about 250 tapes have been processed on the ACP II system. This consists of three farms with 16, 16, and 11 ACP II nodes each. Stripping has not yet commenced there. At present, OSU reconstructs and strips events and sends tapes to FNAL, while U. Miss. sends the DST's to FNAL for stripping. Strips are duplicated at FNAL and mailed to other institutions. Release 6 of the offline software will allow multiple output streams. We decided to implement a dual stream strip with raw data included, producing about 8 tapes per run. Work has been done to improve DST unpacking speed, add new code for the muon wall, change electron probability cuts, and improve our efficiency for lambdas in region 2 (inside and after the first analysis magnet), and improve the magnetic field parameterization.

Tufts has 3 Silicon Graphics machines (two 4D/25's and an Indigo) which are being used to run the E791 software. Our total of about 56 MIPS (million instructions per second) is small compared to a 1000 MIPS farm, but it is still possible to reconstruct samples of data from specific strips and to analyze DST's produced by the farms.

The CMS program (Code Management System) on the Fermilab Physics Department VAX cluster continues to provide centralized storage for our source files and libraries. Nightly updates are shipped via Internet to remote machines, including one of the $4 \mathrm{D} / 25$ machines at Tufts. A high-speed network connection, with very little down-time and good interactive response, is essential for this system to function well.

\section{C.4. Current work at Tufts}

Prof. Milburn is working to improve understanding of the field map of the two analysis magnets in the Tagged Photon Spectrometer. He continues to check the behavior of the magnetic field values at various points along the analysis chain in order to make sure that significant errors have not been introduced in the process, and to refine the field-evaluation procedures when possible. It recently became apparent that some tracks were being lost in the region near the edges of the second magnet which were outside the range of the "ziptrack" data. It seems likely that tracking could be improved by either extrapolation of the central field in some way, or by modeling the entire magnet in sufficient detail to 
produce agreement of the predicted magnetic field with that of the well-measured central region. Our colleagues at the University of Cincinnati are also studying the field mup and its effects on the efficiency of track finding, particularly in regard to neutral strange particles ( $K_{s}^{0}$ 's and $\Lambda$ 's).

Prof. Napier has continued work on the offline event display program. This program was generalized last year to run with several lower-level graphics packages on the various machines (VAX, IBM RIOS, DECstation 5000, and SGI) available in the collaboration. The CERN package, HIGZ, now serves as a graphics interface, and basic graphics primitives are provided by commercial graphics packages such as GKS, GKS-3D, DI-3000, PHIGS, GL, or X11. Most of the E791 collaborators have one or more of these packages available. The X11 libraries may be obtained free of charge over the Inter.⿲et, and this is the package of choice on most UNIX machines we are using. It is likely that some bugs still exist in this software, and the availability of a functionally-equivalent display program linked with another graphics package has proven extremely helpful in tracking down such problems. Juan Astorga has also contributed to this work by modifying and extending the E769 version of the display program to examine data for his thesis.

\section{C.5. Summary}

E791 has now reconstructed about $20 \%$ of the 20 billion events on tape. The entire data ser is estimated to yicld in excess of 200,000 reconstructed charm decays for detailed study. Preliminary analysis of the already large data sample indicates lifetime values for $D^{0}$ and $D^{ \pm}$which are quite close to the Particle Data Group values. However the discrepancy is large enough to wariart further study. The present data give a limit of about $2 \%$ for mixing of $D^{0}-\overline{D^{0}}$, and this is espected to decrease as the analyzed sample becomes larger. Four large farrms of computers are now in operation at OSU, U. Miss , Fermilab, and CBPF (Brazil). Tufts physicist,s are continuing to work on projects which will help insure the quality of the reconstructed data. We look forward to physics analysis of DST's at Tufts during the next several years. 

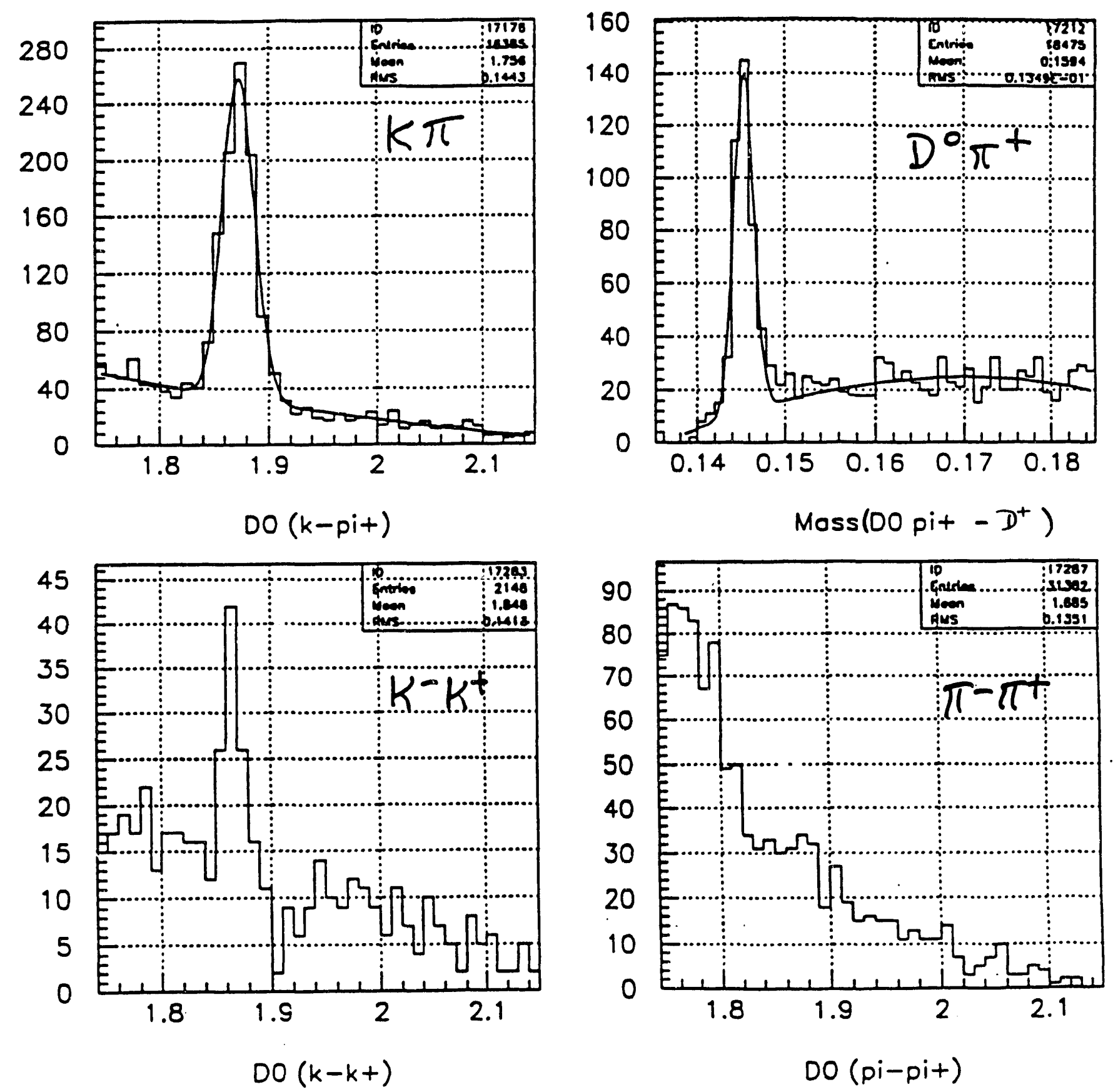

Figure 3.C.1.1 

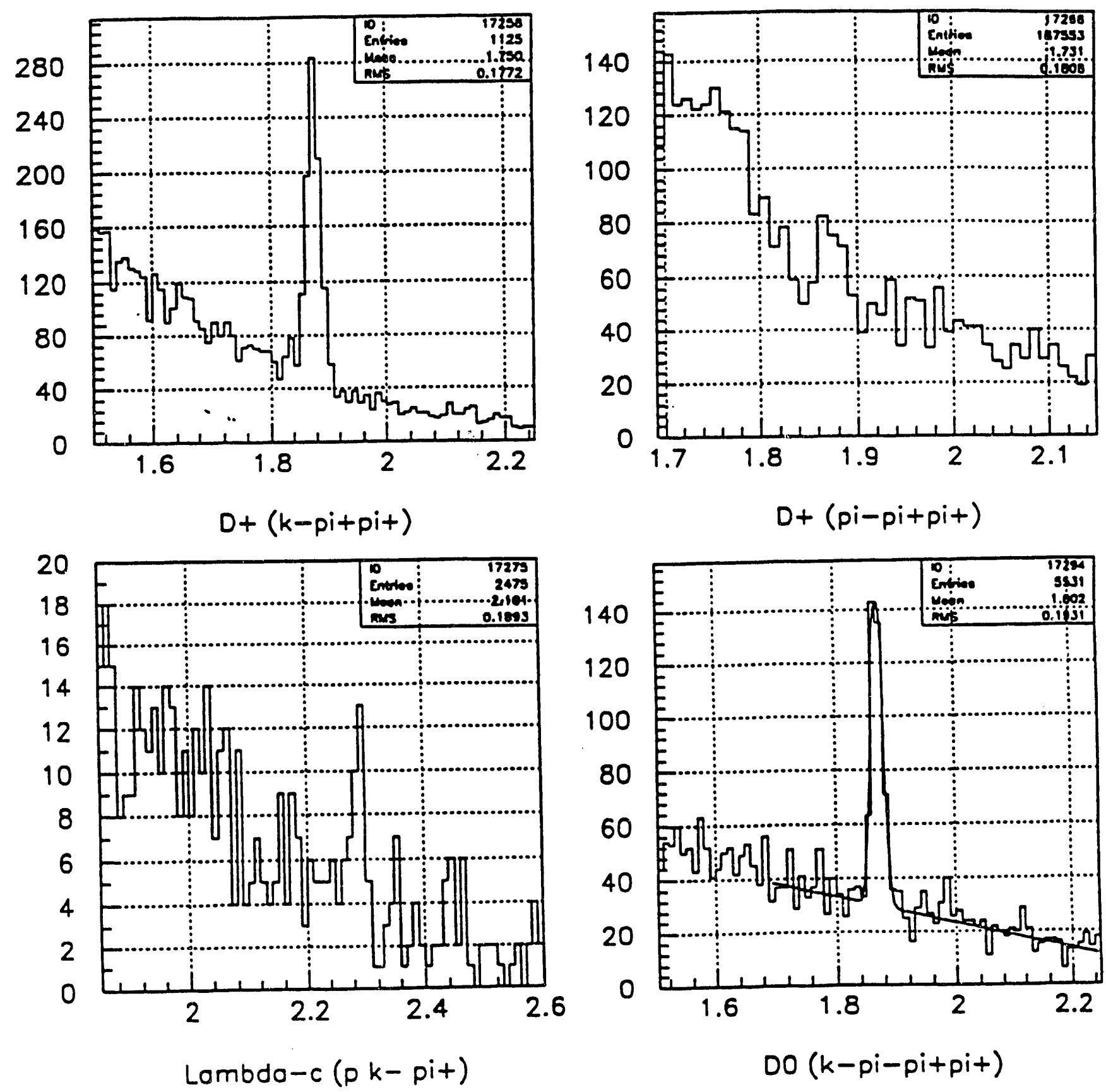

Figure 3.C.1.2 


\section{D. Neutrino oscillations at the Fermilab Main Injector}

\section{D.1. Introduction}

The question of neutrino oscillations has been tantalizing particle physicists for over 20 years. Recent results on solar neutrinos and atmospheric neutrinos, as well as theoretical ideas such as the seesaw model and hot dark matter, suggest that the tau-neutrino may have a mass in the range 0.1 to $30 \mathrm{eV}$. It is also suggested that the mixing angles between neutrino flavors are comparable to those between quark flavors. The atmospheric neutrino data on the $\nu_{\mu} / \nu_{e}$ ratio could be explained by $\nu_{\mu} \rightarrow \nu_{\tau}$ oscillations. However, these data in themselves are unlikely ever to provide convincing evidence of neutrino oscillations because of the need to rely on comparisons with models that have large uncertainties. A convincing model-independent result can come from an accelerator experiment in which a beam of neutrinos of one flavor is made, this flavor disappears in part, and a different flavor is seen to appear. If the masses and mixing angles are as suggested above then an intense $\nu_{\mu}$ beam at the Fermilab Main Injector would provide an excellent opportunity to discover oscillations. The Tufts group is involved in two proposals to explore these regions using a Main Injector neutrino beam. The short baseline experiment, P803, would explore the range of $\nu_{\tau}$ mass greater than $1 \mathrm{eV}$, reaching mixing angles as low as $\sin ^{2} 2 \theta=10^{-5}$. A long baseline experiment, P822, in which the beam would be directed at the Soudan II site in northern Minnesota, $735 \mathrm{~km}$ from Fermilab, could explore the mass region from $\sim 0.1$ to a few $\mathrm{eV}$ for large mixing angles. This is the region suggested by the atmospheric neutrino data. Considerable progress has been made at Fermilab on the beam design, including the method of extraction so that it can be aimed at Soudan II. An initial conceptual design report was prepared over a year ago and the work is continuing. In addition to these two Main Injector proposals, a third proposal, P872, has been submitted for a beam dump experiment at the Tevatron to search for $\nu_{\tau}$ 's coming from $D_{s}$ decay. This experiment would precede $\mathrm{P} 803$ but would use the same apparatus, thus demonstrating the ability of P803 to detect $\nu_{\tau}$ interactions. It would also be a significant discovery experiment confirming the existence of the $\nu_{\tau}$.

\section{D.2. Short baseline proposal (P803)}

(Profs. Kafka, Napier, Oliver and Schneps, with collaborators from Greece, Israel, Japan, Korea, and U.S.A.)

The short baseline experiment P803 is designed to detect $\nu_{\mu} \rightarrow \nu_{\tau}$ oscillations via the appearance of $\nu_{\tau}$ charged current interactions in a $\nu_{\mu}$ beam. These interactions are found by detecting the decay of the produced $\tau^{-}$lepton. P803 uses nuclear emulsion to achieve the very fine spatial resolution required to detect the $\tau^{-}$decay vertex. Roughly one half ton of nuclear emulsion serving as both target and vertex detector will provide an adequate number of events. Additional elements of the experiment are scintillating fibers, wire chambers, an electromagnetic calorimeter, a hadron calorimeter, and a magnet for momentum determination (see Fig. 3.D.1). During the past year our group has undertaken three investigations related to $\mathrm{P} 803$ :

(i) Scintillating glass fibers. The purpose of the scintillating fibers in P803 is to precisely locate neutrino events in the nuclear emulsion. Success with present plastic-fiber 
technology has been limited. The signal is small so the fibers cannot be made less than about 500 microns in diameter. Profs. Oliver and Schneps, together with Prof. J. Goldberg of Technion (Israel), and Prof. E. Snitzer of the Fiber Optics Institute of Rutgers University, have been looking into the use of cerium loaded glass fibers. Prof. Snitzer, a world expert in glass fibers, believes it would be possible to produce such fibers giving a larger signal than the present plastic fibers, thereby making smaller diameters possible. The fibers would also be more durable than plastic ones. Fermilab has provided $\$ 20,000$ in $R \& D$ funds to pursue this work.

The Fermilab funds are being used to enable the Rutgers Institute to produce several "preforms" of cerium loaded glass samples by a technique known as the vapor deposition method. The host will be a highly transparent fused silica glass loaded with trivalent cerium as the scintillating agent. The cerium fluoresces with a $50 \mathrm{~ns}$ lifetime. Other dopants which improve the performance of the glass will be varied in the different samples. These samples will be tested at Tufts, Technion and Rutgers to determine their optical properties and response to radioactive sources of alpha and beta particles. Beam tests are scheduled at CERN for August 1993. The first preliminary sample has now arrived at Tufts and we are preparing to study its response to alpha particles. Several additional samples are expected before the August beam tests.

(ii) Measurement of $\delta m^{2}$. Profs. Oliver and Schneps, and Research Associate Willocq, have investigated how well one can measure the $\nu_{\tau}$ mass in P803. If $\nu_{\mu} \rightarrow \nu_{\tau}$ oscillations occur, the energy distribution of the $\nu_{\tau}$ events will depend on the $\nu_{\tau}$ mass. To investigate this the present beam design was used to generate $\nu_{\tau}$ appearance events assuming various masses. The energies of the generated events were then used in a maximum likelihood fit where the $\nu_{\tau}$ mass was the free parameter. The result was quite interesting. We found that with only 5-10 events we can determine the mass to about $10 \%$ if it is $10 \mathrm{eV}$. But for the interesting region of about $20 \mathrm{eV}$, suggested by cosmology, we can not determine it at all. This is because at $10 \mathrm{eV}$ the beam length is about one oscillation length, whereas at $20 \mathrm{eV}$ the beam length corresponds to 4-5 oscillation lengths. In this case the 200 meter length of the decay tunnel washes out our resolution. A similar washing out occurs if the mass is much below $10 \mathrm{eV}$. However we found that by reducing the beam decay tunnel length by a factor of two to four we can improve the resolution considerably without sacrificing too much in beam intensity. These considerations will play a role in the beam design now proceeding at Fermilab.

(iii) Identifying $\nu_{\tau}$ events kinematically. Charged-current $\nu_{\tau}$ events where the $\tau$ decays hadronically will look like neutral curren events when the $\tau$ decay vertex is not seen. Therefore all apparent neutral current events would have to be scanned in order to find the few $\tau$ events. It would take about three years to scan the 50,000 neutral current events expected. We investigated whether the use of a multivariant discriminant analysis, using over 20 kinematic variables to start with, could eliminate from consideration a large fraction of the neutral current events which needed to be scanned. The result of the analysis on Monte Carlo generated events, carried out by Dr. Willocq in consultation with Professors Kafka and Schneps, was that we could indeed reduce the number of events to be scanned by a factor of at least two, thus reducing the scanning time for the experiment to about 18 months or less. 


\section{D.3. Soudan II long baseline proposal (P822)}

(Profs. Kafka, Mann, Milburn, Napier, Oliver, and Schneps, with collaborators from Argonne, Minnesota, Oxford, and the Rutherford Appleton Laboratory.)

The original P822 proposal envisioned using the 1 kton Soudan II proton decay detector together with a similar near detector of about 50 tons. Since then considerable study has gone into the design of a coarser grained detector of $\sim 5-10$ ktons to supplement the 1-kton detector at Soudan. The increase in the event rate would greatly enhance the sensitivity of the experiment. Three methods are proposed for detecting oscillations:

(1) The beam flux in the near and far detectors is compared. This is a pure $\nu_{\mu}$ disappearance experiment.

(2) Muons produced in the rock in front of the far detector are measured to yield the $\nu_{\mu}$ content of the beam. Neutrino events of all flavors, both NC and CC, are counted in the central detector. These are a measure of the total beam flux. If oscillations occur, the $\nu_{\mu}$ content of the beam would be less than expected.

(3) The NC/CC ratio is measured at the far detector. Since $83 \%$ of $\nu_{\tau}$ CC events appear to be $\nu_{\mu} \mathrm{NC}$ events, oscillations would give an apparent $\mathrm{NC} / \mathrm{CC}$ ratio greater than the standard value of 0.31 . The rreat advantage of this method is that it is independent of the beam flux, which could be uncertain to $\pm 10 \%$. A similar near detector would be used as a calibration device. It should measure $\mathrm{NC} / \mathrm{CC}=0.31$, after the usual background corrections.

During the past year the Tufts group has carried out several studies related to the long baseline experiment:

(i) Neutrino beam geodesy and control. Prof. Milburn has continued to look into technical problems connected with aiming and sending a high energy neutrino beam 735 $\mathrm{km}$ from Fermilab to the Soudan mine in northern Minnesota. Knowing the required direction and dip-angle for the beam is a difficult but tractable surveying problem; actually sending a high-intensity horn-focussed neutrino beam along this precise direction - and continuously monitoring it to prove one has done so - is another. The practical difficulties of on-line monitoring with short-term feedback to the controlling operators are readily evident when one considers that the mere "detection" of a neutrino beam can be in itself a major experiment!

To provide a basis for understanding the "optics" of the proposed neutrino beam Prof. Milburn has established at Tufts both the primary neutrino-beam simulation package NUADA, used at Fermilab, and also a somewhat simpler and more usable Monte Carlo system, called NEUBM, which was developed by Prof. Noel Stanton at Ohio State University specifically for the proposed P803/P822 experiments. Both of these simulators generate a spectrum of secondary hadrons from primary Tevatron protons incident on a small target, propagate these hadrons through the magnetic optics of the focussing horn system, and then allow the mesonic component to decay into the desired neutrinos. Neutrino intensities, energies and directional characteristics are thereby estimated. The practical difficulty vis-a-vis P822 is that the spatial aperture of the horn - about $30 \mathrm{~cm}-$ governs the "near field" distribution of neutrinos in the beam at Fermilab, while it is the 
directional distribution of the neutrino momenta which governs the "far field" distribution of the beam - of the order of a kilometer in size - at Soudan. Thus, practical monitors in the "near field" do not necessarily give a clear indication of what is happening in the "far field," and this latter information is important insofar as the neutrino energy distribution depends significantly upon their angles of emission from the parent mesons. Preliminary simulations have been made using the nominal magnetic horn geometries of the early P822 proposal; further work is anticipated as the beam-line engineering studies are developed at Fermilab.

(ii) Design studies for a 5-10 kton detector at Soudan. For a long baseline experiment such as proposed in P-822, Soudan-II offers the advantages of being an existing detector of fine granularity. Unfortunately, its mass of one kiloton sets a lower limit on the value of $\sin ^{2} 2 \theta$ that can be reached to $\sim 0.05$. The ultimate limit (probably $\sim .01$ ) is imposed by systematic errors in the variation of the beam composition and energy with distance from the source. To reach this limit a more massive detector is clearly required. ${ }^{1}$ Three designs for tracking elements and architecture for a 5-10 kiloton detector, to be located in the Soudan-II cavern downstream along the neutrino direction from Fermilab, have been put forward by the Oxford group, the Rutherford group and by Prof. Oliver for our group. The three designs share the view that the optimal detector is a planar iron tracking calorimeter which provides track sampling for every $2 \mathrm{~cm}$ (or less) of iron traversed. Rutherford Lab is investigating resistive plate chambers (RPC's) as the detecting elements, and Oxford is investigating drift tubes with shaped cathodes. Tufts is taking the view that current tracking technology may be more than adequate and that the detector should be built with simple rugged components.

In the Tufts design, the cavern-filler detector consists of $2 \mathrm{~cm}$ thick steel plates interleaved with thin-walled aluminum proportional tubes as shown in Fig. 3.D.2. The choice of proportional tubes for the detection elements is motivated by the desire to measure the visible energy in the events to be able to recognize tau-appearance effects. The choice of aluminum tubes is to take advantage of the intrinsically straight and clean nature of extruded aluminum to facilitate detector construction. The cross section of the proportional tube (a $3 \mathrm{~cm}$ wide tube is shown in Fig. 3.D.3) is rectangular with walls made as thin as possible to minimize the material costs of the aluminum. The long $(7.6 \mathrm{~cm})$ dimension of the rectangle is short enough to provide adequate spatial resolution, to provide adequate response time $(5 \mu \mathrm{s})$, and to allow wall thickness of less than $1.3 \mathrm{~mm}$, yet not so short that an excessive number of detection electronic channels is required. It is desireable to make the width of the proportional tube to be as narrow as possible. A 8-m long prototype tube of $3 \mathrm{~cm}$ width was built at Tufts and tested in our cosmic-ray test stand. The tube displayed an efficiency plateau with operating voltage as shown in Fig. 3.D.4. The response time of the tube was also measured and found to have the satisfactory features shown in Fig. 3.D.5. With 3-cm wide tubes, the mean density that can be achieved in the detector is $3.3 \mathrm{gm} / \mathrm{cm}^{3}$. There would then be 1 kton of material for every $6.2 \mathrm{~m}$ of length in the Soudan cavern or a maximum tonnage possible of roughly $4 \mathrm{kton}$. We are investigating just how narrow can an $8-\mathrm{m}$ long tube be made and still have the sense wire (supported at just the two ends) be stable against being pulled to the wall. For this purpose we have constructed an "adjustable-gap" tube using four of the $3-\mathrm{cm}$ wide extrusions available to 
us. We have found that the tube could be made as narrow as $2 \mathrm{~cm}$ or possibly $1.8 \mathrm{~cm}$. With a width of $2 \mathrm{~cm}$ the density of the detector would be $4.1 \mathrm{gm} / \mathrm{cm}^{3}$ and there would be $1 \mathrm{kton}$ of material for every $5.3 \mathrm{~m}$ of detector length. The total mass could then be $5 \mathrm{kton}$ and there would be improved muon identification because of the greater density. There is a total of 12,000 proportional tubes per kiloton. Since deep underground there is near absence of background within the duty cycle of the neutrino beam we can use time multiplexing to further reduce the number of detector channels. We require analog information from each tube. We would design a preamp for each tube that would have a decay time of $8 \mu \mathrm{s}$ to allow the sampling of the amplifier output at that interval. A FET analog switch would be used to step at $1 \mu$ s intervals through the tubes in groups of 8 and direct these outputs to a flash ADC. The downstream electronics would then need to process only 1500 channels per kiloton.

\section{D.4. Discovery of $\nu_{\tau}$ charged current interactions (P872).}

(Profs. Kafka, Napier, Oliver, and Schneps, with collaborators from Greece, Japan, and the United States.)

The object of proposal P872 is to discover $\nu_{\tau}$ charged current interactions by detecting the $\tau$ lepton produced in them. However in this case the $\nu_{\tau}$ 's would be produced in a beam dump using the $800 \mathrm{GeV}$ proton beam at the Tevatron. $D$, mesons are produced in the dump and decay with a $3-5 \%$ branching ratio to $\nu_{\tau} \tau$. The susequent decay of the $\tau$ yields another $\nu_{\tau}$ so that two $\nu_{\tau}$ 's are produced per $D_{s}$. The method of detection is exactly the same as that proposed in P803, and in fact it is proposed that the P803 detector be adapted to this experiment, but stretched out to account for the higher energy neutrinos. The tentative experimental design has the emulsion target located about 25 meters from the dump and it is estimated that about $200 \nu_{\tau}$ events would be detected in a 9 month run. The main challenge for such a design is sweeping the intense flux of muons emerging from the dump away from the emulsion face. Monte Carlo calculations using an existing Fermilab magnet (SELMA) now in the PW8 hall indicate that this is possible, but experimental tests would be desirable. P872 is now in the form of a Letter of Intent. A fuller proposal will be developed over the coming months. If the proposal is approved, a likely running period would occur about 1996, a few years before P803 would run.

\section{D.5. Status of the three proposals}

The three proposals were recently (June 7-9, 1993) presented to a special subcommittee of the Fermilab PAC. They will be reviewed during the Aspen meetings this summer and then be considered again at the PAC deliberations in November. We believe that $\mathrm{P} 803$ is likely to be approved at that time and that P872 will be encouraged to proceed with sweeping magnet tests. Approval for P822 will probably be delayed until it adopts a sp: fic design for the 5-10 kton detector and shows that the neutrino beam characteristics at :e mine are sufficiently well understood to achieve the sensitivity proposed. These studies will be pursued in the coming months. 


\section{Section 3.D References}

1. P. J. Litchfield, Proceedings of the Workshop on Long-baseline Neutrino Oscillations (Fermilab, Nov. 1991) ed. M. Goodman (Fermilab 1992) p. 387. 


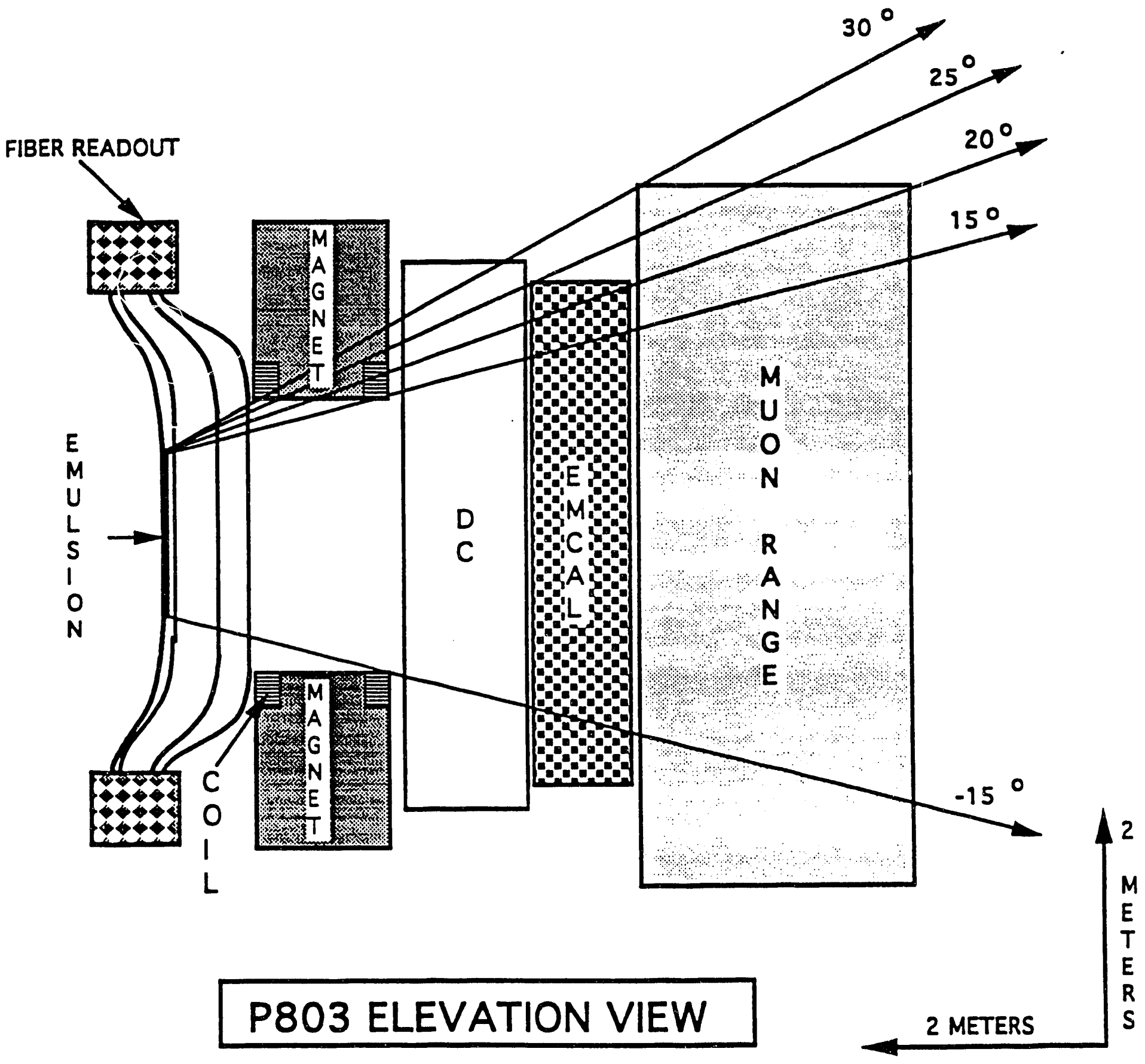

EMULSION $1.4 \times 1.8$ METERS

Figure 3.D.1. The layout of the short baseline experiment.

The readout of the horizontal fibers is not shown. 

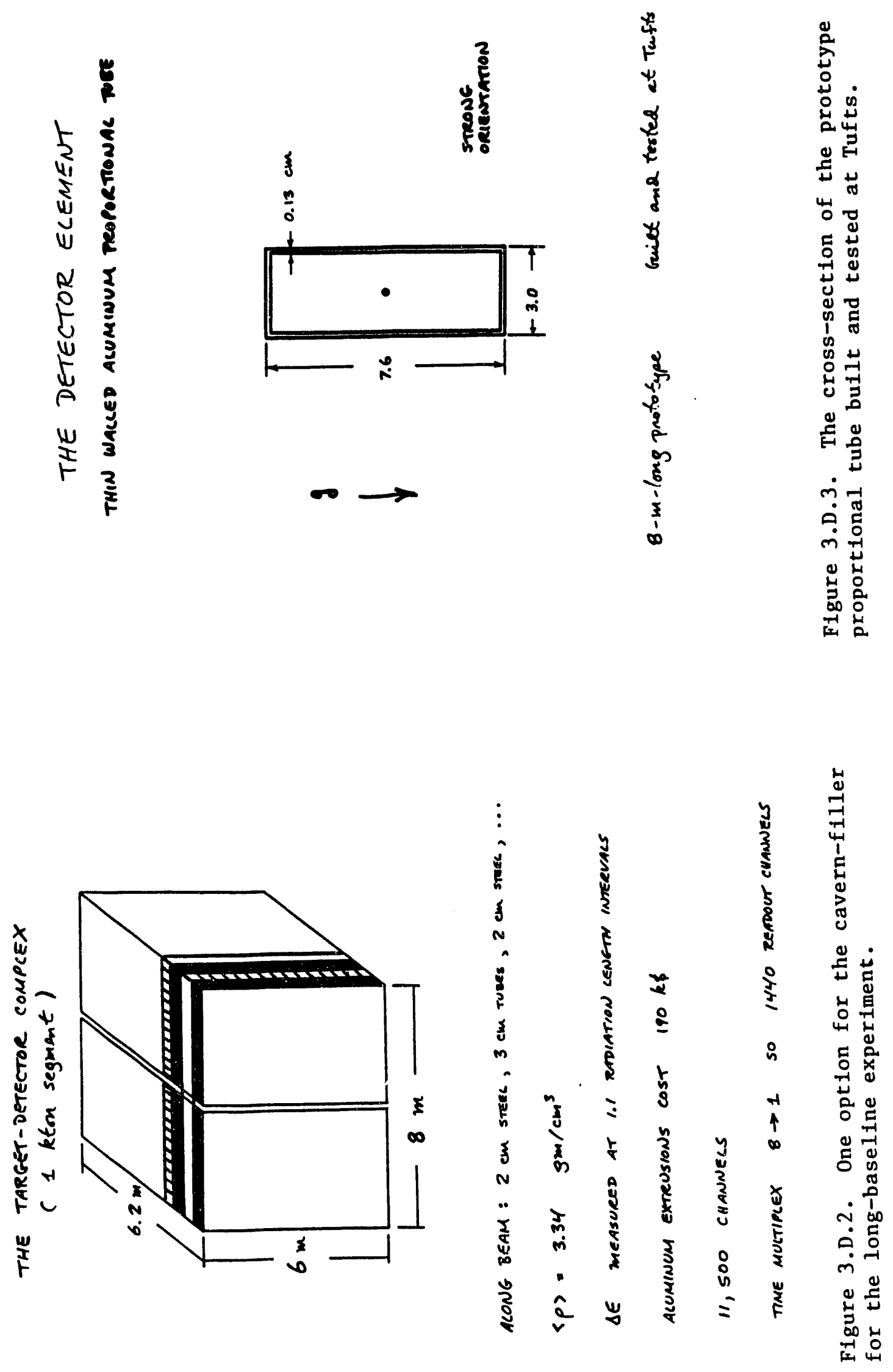


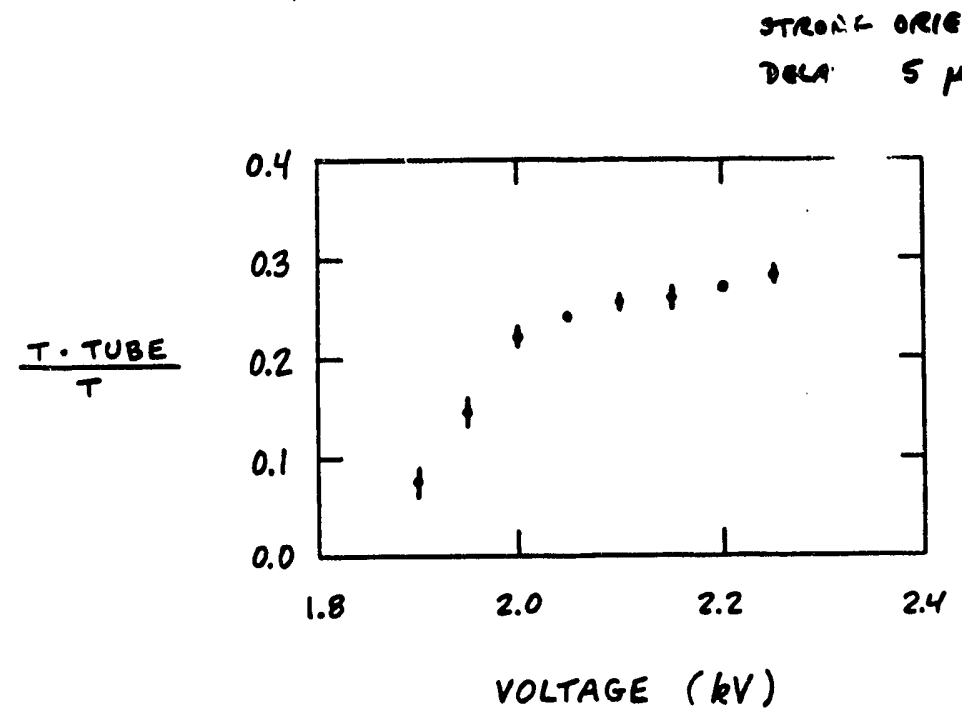

Figure 3.D.4. The dependence of the efficiency of the prototype tube on operatirg voitage.

$2.2 k V$

weAK ORIENTATION

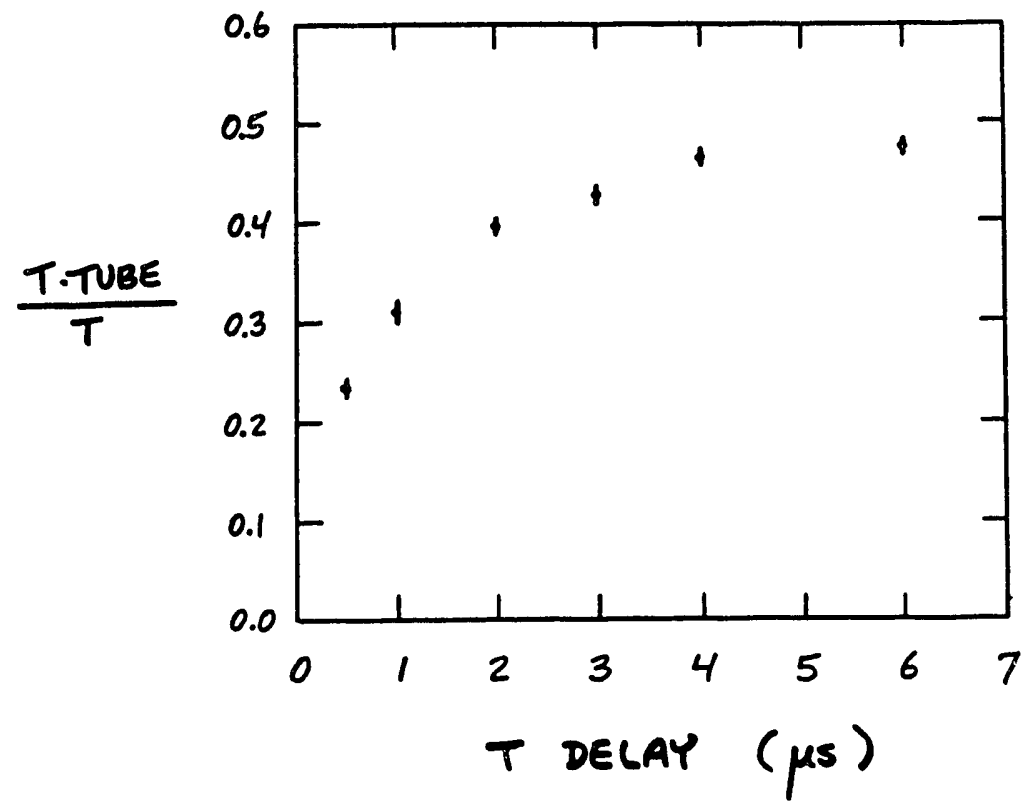

Figure 3.D.5. The response time of the prototype tube. Full efficiency is achieved after $4 \mu \mathrm{s}$. 


\section{Soudan-II nucleon decay project}

(Profs. Kafka, Mann, Milburn, Napier, Oliver, Schneps; Graduate Research Assistants Ewen, Leeson, and Sundaralingam; Teaching Assistants Pfenning and Wall; with collaborators from Argonne National Laboratory, Minnesota and Oxford Universities, and the Rutherford-Appleton Laboratory.)

\section{A. Introduction}

The "population" of large underground physics experiments which will be reported or cited at conferences this summer, is large enough to exhibit an evolutionary sequence. Dead and dismantled, but still glowing in terms of new analyses reported, ${ }^{1-3}$ are the IMB-3 and Frejus nucleon decay search experiments. The Kamiokande water Cherenkov detector continues to take data, though the collaboration's focus is shifting to the task of completing and turning on Super-Kamiokande by mid-year 1996. Soudan-II reported its first contained event results last autumn; by the end of 1993 it will match Kamiokande's operating fiducial tonnage while providing high resolution imaging capability for non-relativistic as well as relativistic tracks. With regard to observations of interest concerning nucleon decay and atmospheric neutrinos, Soudan is a good bet to dominate the field during the next three to five years. The Gran Sasso experiments MACRO and LVD may also contribute to atmospheric neutrino study in the near future. During the year past, MACRO reported new results on underground multiple muon events, ${ }^{4}$ while a study of neutrino-induced upward going muons is in progress. A premier detector at the Gran Sasso site will be the long-drift liquid argon tracking calorimeter ICARUS. Tracks imaged with a two-ton prototype device are observed with bubble-chamber-like quality; the spatial resolution is about 150 microns. ${ }^{5}$ The proposal is to build a 3000 ton device, and so the scale-up from the prototype represents a daunting technological challenge.

Among all recent analyses from the underground experiments, it is the anomalous ratio of $\nu_{\mu}$ to $\nu_{e}$ interactions induced by atmospheric neutrinos which has dominated the limelight during the past year. The effect is "one of the few tentative pieces of evidence for physics beyond the Standard Model". ${ }^{6}$ Measurements of anomalously low values for this ratio have emerged during the past five years in the large exposures accumulated by the Kamiokande and IMB-3 water Cherenkov experiments. In those experiments, the essential observation involves relative rates among subsamples of contained events whose images appear either as single, sharply-defined rings (muon-like), or as single, diffuse rings (electron-like). The majority of single-ring events are supposedly quasi-elastic charged current interactions. We take the ratio of $\mu$-like to e-like single-ring event rates $N_{1}(\mu)$ and

$N_{1}(e)$ to measure $R_{\nu}$, the muon neutrino flux, $N\left(\nu_{\mu}+\bar{\nu}_{\mu}\right)$, divided by the electron neutrino flux, $N\left(\nu_{e}+\bar{\nu}_{e}\right)$. From the decay chains of mesons and muons in cosmic ray showers, it is expected that $R_{\nu}$ should be nearly 2.0. This expectation is borne out in all detailed calculations of the atmospheric neutrino flux to date. ${ }^{7}$ However, in the most recent reports by Kamiokande and IMB-3 the observed ratio is about 1.2.

Experimental results are usually expressed in terms of the ratio-of-ratios, $R_{\nu}^{\prime}$ :

$$
R_{\nu}^{\prime}=\frac{\left(\nu_{\mu} / \nu_{e}\right)_{\text {measured }}}{\left(\nu_{\mu} / \nu_{e}\right)_{\text {predicted }}}
$$


with $R_{\nu}^{\prime}$ found to be less than 1 . For the water Cherenkov experiments,

Kamiokande (6.18 kiloton years): $R_{\nu}^{\prime}=0.60 \pm 0.07$, and

IMB-3 (7.7 kiloton years): $R_{\nu}^{\prime}=0.54 \pm 0.07$.

The $R_{\nu}^{\prime}$ value is more than four standard deviations below unity for either experiment. A new $R_{\nu}^{\prime}$ measurement obtained with our Soudan-II fine-grained iron tracking calorimeter was reported in November 1992 (see Section 4.B.1). The Soudan value supports the view that $R_{\nu}$ is anomalous and does not represent an instrumentation effect with the water Cherenkov experiments.

To interpret the low $R_{\nu}$ value, one needs to know whether the $\mu$-like events of the numerator are too few, whether the e-like events of the denominator are too numerous, or whether a combination of both circumstances is actually the case. Two points of view have been expressed in the recent literature. According to one view (Refs. 8,9), there is an apparent dearth of muon-neutrino events (accompanied perhaps by a mild excess of electron-neutrino events (Refs. 10,11)), which is interpreted as evidence that neutrino oscillations deplete the muon-neutrino flux over distances of 10 to 10,000 kilometers. A different view, published by Tufts researchers in September 1992, assumes that the expected number of $\nu_{\mu}$ events has been detected, and that there is an excess of electron-like events that requires interpretation. We attribute the excess of electron-like events to proton decay via the mode $\mathrm{p} \rightarrow \mathrm{e}^{+} \nu \nu$, with a lifetime of $4 \times 10^{31}$ years. ${ }^{12}$ An updated version of the case to be made for nucleon decay was presented by us at the American Physical Society DPF meeting at Fermilab during November and is described in Section 4.B.2 below. ${ }^{13}$

\section{B. Status of the Soudan-II experiment}

Soudan-II is designed to detect the spontaneous decay of protons or bound neutrons predicted by Grand Unified Theories (GUTs) of the strong, weak, and electromagnetic forces. The experiment is optimized for decay modes producing charged $\mathrm{K}$ mesons, predicted by the supersymmetric GUTs which are favored by LEP results, and for other complicated modes which are difficult to analyze in the large water Cherenkov detectors. Ionization measurements and bubble-chamber-like event reconstruction allow Soudan to distinguish between nucleon decay and the neutrino interactions which can mimic it.

By December 1992, a team at Argonne National Laboratory put the finishing touches to the 227th 4.3-metric-ton calorimeter module for the Soudan-II nucleon decay detector. This completed the fabrication of the steel and plastic drift chamber modules which began at Argonne and at Rutherford Laboratory in 1985. Module components were constructed by the Minnesota and Oxford groups, while Tufts University built the active shield of proportional tubes which now surrounds the massive underground tracking calorimeter.

As of June 1993, 860 tons of Soudan II are in routine operation, with the detector scheduled to reach its final mass of 963 tons by this autumn. The experiment has been acquiring data since the first 250 tons came into full operation in 1989. About 1.3 fiducial kiloton years of exposure has been recorded for nucleon decay and cosmic ray neutrinos. A sample of 15 million cosmic ray muon tracks is also being studied to learn about the nuclear composition of cosmic ray primaries. The latter work is aided by a surface detector which 
measures the energies of the parent air showers for a subset of the underground events. The collaboration has presented an initial measurement of atmospheric neutrino interactions from the first half kiloton year exposure. Although the statistics are limited, the resulting ratio of muon to electron neutrino interactions appears to support the anomalously low value obtained by the IMB and Kamioka. de experiments. The Soudan neutrino sample will soon be doubled when analysis of data already in hand is completed.

One popular explanation of the unexpectedly low muon to electron neutrino ratio is that muon neutrinos oscillate into tau or electron neutrinos as they travel from their production poir $i$ in the atmosphere. The Soudan collaboration has proposed to search directly for neutrino oscillations using a $730 \mathrm{~km}$ long neutrino beam from Fermilab. A high intensity $20 \mathrm{GeV}$ muon neutrino beam produced by the new Fermilab Main Injector would be aimed towards Soudan at a $3.3^{\circ}$ angle below the horizon. If the atmospheric neuirino result is due to oscillations, many of the muon neutrinos in the Fermilab beam would become tau or electron neutrinos on their trip to Soudan. This will decrease the fraction of the expected 900 interactions per year containing final state muons, and provide an unambiguous signal of neutrino oscillations. Attractive features of this proposal are both the existence of the operating Soudan II detector and the possibility of building a new, more massive detector at the same site. When the U.S. Department of Energy approved the cavity excavation in 1983, they allowed for future follow-on experiments by funding a laboratory large enough for a second large detector in addition to Soudan II. Several ideas for a new detector for the long baseline experiment are now being developed. Details of our long baseline neutrino oscillation proposai to Fermilab (P-822) are presented in Section 3.D.1.

\section{B.1. Measurement of the atmospheric neutrino flavor ratio at Soudan}

A total of 1.3 kiloton years of data has been recorded and the first $0.5 \mathrm{k}$ iy has been analyzed. Processing of 17 million triggers yielded 25,000 possible contained event candidates which were scanned by physicists. Containment is defined as no track coming closer than $20 \mathrm{~cm}$ to the outside of the detector. The next step was to classify the topology of the contained events as single track, single shower, or not being due to a quasi-elastic interaction (non-QE). The single track and single shower events are mostly (72\%) quasi-elastic interactions, according to our Monte Carlo simulation. In this separation, an observed recoil nucleon or muon decay was ignored. Events which appeared to be inelastic or neutral current interactions were classified as non-QE.

Certain kinds of noise in the deiector can appear to be similar to low energy showers. We have identified and eliminated sucil events. Nevertheless at this stage we have applied an additional ._quirement that showers must have more than $200 \mathrm{MeV}$ of visible energy.

Contained events with shield hits are due to interactions of neutral particles produced by muon interactions in the surrounding rock. A study of such events indicates that the efficiency of detecting at least one charged particle passing through the active shield is high. The shield etficiency and random rate are continuously monitored using the $0.2 \mathrm{~Hz}$ of through-going muons. The final sample contains 38 no-shield-hit (neutrino candidate) events and 23 one-shield-hit events which we use to estimate the background. In the 
no-shield-hit events we identify 13 tracks, 14 showers, and 11 non-QEs. In the one-shieldhit sample there are 16 tracks, 5 showers, and 2 non-QEs. The neutrino event counts have been corrected for losses due to random shield hits and contamination due to shield inefficiencies, giving a final sample of 11.0 tracks, 13.9 showers and 1.5 non-QEs.

In a Monte Carlo exposure of 3.17 kton-years, analyzed in an identical manner, we identified 20.8 tracks, 14.5 showers, and 16.9 non-QE events, after normalizing to our exposure. Scanners correctly identified $96 \%$ of the simulated single tracks and showers.

The track/shower ratio is $11.0 / 13.9=0.79 \pm 0.39$ (stat). The Monte Carlo ratio is 1.43. Major systematic uncertainties at this stage are $15 \%$ for the Monte Carlo simulation, $14 \%$ from the statistics of the Monte Carlo, and $6 \%$ from the uncertainty in shield efficiency. Combining these errors in quadrature, we obtain:

$$
\begin{aligned}
R^{\prime} & =\frac{(\text { track } / \text { shower })_{\text {measured }}}{(\text { track } / \text { shower })_{\text {predicted }}} \\
& =0.55 \pm 0.27(\text { stat }) \pm 0.10(\text { sys })
\end{aligned}
$$

\section{B.2. Interpretation of the atmospheric neutrino ratio anomaly}

The situation with the low atmospheric neutrino flavor ratio could be clarified if the absolute fluxes for all neutrino flavors $\left(\nu_{\mu}, \bar{\nu}_{\mu}, \nu_{e}, \bar{\nu}_{e}\right)$ were accurately known - which, however, is not the case. The neutrino oscillation interpretation of Refs. 8,9 is based upon the absolute neutrino fluxes calculated by Gaisser et al. (Ref. 14), which happen to be the highest in the literature. In the initial formulation of our proton decay scenario, we utilized absolute atmospheric neutrino fluxes calculated by Bugaev and Naumov ${ }^{15}$, which are distinctly lower than the Gaisser et al. fluxes. Subsequently we reformulated the argument in a way which does not rely on any neutrino flux calculation. We assume that no atmospheric muon neutrinos are lost due to oscillations or other processes.

In Fig. $4.1 \mathrm{a}$ we show the distribution of lepton momentum for all contained, muonlike single-ring events the $6.18 \mathrm{kty}$ exposure of Kamiokande. The detection efficiency for these events is shown superimposed. The corresponding distribution for electron-like single ring events is shown in Fig. 4.1b. The Cherenkov threshold for detection of muons in water precludes direct observation of events with muon momenta of $100-200 \mathrm{MeV} / \mathrm{c}$. For this bin we have inferred a counting rate by extrapolating plausible distribution shapes. The shapes were calculated using muon quasi-elastic events and were fitted to the observed distribution of $\mu$-like events. We regard the $\mu$-like events to be free of any "new physics". Also, we assume that neutrino cross sections are nearly the same for corresponding $\nu_{\mu}$ and $\nu_{e}$ charged current channels. Then we can use the number of $\mu$-like events in each momentum bin, together with the experimental detection efficiencies and the e-like to $\mu$ like event ratio $1: 2$, to predict the number of e-like events to be observed in the absence of new physics. The expected e-like event rates are depicted by the open triangles in Fig. 4.1b. We observe in Fig. 4.1b that there is an excess of e-like events over and above the expectation inferred from the $\mu$-like event distribution and conventional neutrino fluxes. We note that the excess is significant only in the interval $100-500 \mathrm{MeV} / \mathrm{c}$. We now consider 
nucleon decay processes as candidate sources for this apparent localized excess of singlering e-like events.

The data restrict the number of viable nucleon decay modes in two ways: (i) Topology constraint: Single electron showers are detected; any accompanying particles leave no signal in a water Cherenkov detector. (ii) Momentum spectrum constraint: Single electron showers are detected with significant rate in the momentum interval from $100 \mathrm{MeV} / \mathrm{c}$ to $500 \mathrm{MeV} / \mathrm{c}$.

We find that there is no two-body or four-body nucleon decay mode which, alone, can satisfy the electron spectrum constraint. With three-body modes, there is only one which satisfies both the momentum spectrum constraint and the topology constraint. The mode is

$$
\mathrm{p} \rightarrow \mathrm{e}^{+} \nu \nu \text {. }
$$

To examine the extent to which the above mode satisfies the positron spectrum constraint (ii), we subtract bin-by-bin the rate expectation for the e-like events which is based on the observed distribution of single-ring $\mu$-like events. The excess of e-like events thus obtained is plotted in Fig. 4.1c. Superimposed on the histogrammed data is the phase space momentum distribution for positrons originating with decay (1) in a water medium (dashed line). The shape of the positron momentum spectrum of proton decay mode (1) is observed to describe the excess event distribution rather well.

We conclude that there is one nucleon decay mode which satisfies criteria (i) and (ii); that mode is process (1). If we suppose that proton decay via this mode gives rise to an excess of single-ring e-like events which accounts for the anomaly in the atmospheric $\nu_{\mu} / \nu_{e}$ ratio, then we infer from Fig. $4.1 \mathrm{c}$ that $51 \pm 16$ instances of proton decay have occurred in the $6.18 \mathrm{kty}$ exposure of Kamiokande. The lifetime over branching ratio thus implied is $(\tau / B)=3.7_{-1.0}^{+1.7} \times 10^{31}$ years. $^{12,13}$

\section{C. Deployment and operation of the active shield}

The design, construction, and deployment of the 1800 square-meter cavern-liner shield array has been a primary responsibility of the Tufts group since the inception of the Soudan experiment. During July and August 1992, Tufts physicists worked at Soudan with the mine crew to activate new portions of the north wall complex. This work was continued by the mine crew through Fall 1992 and Spring 1993, and so deployment of Tufts-built proportional tube manifolds is mostly complete, with the exception of a few speciallytailored panels which need to be installed at the base of the north wall. Tufts physicists are scheduled to work at Soudan during June and July on manifold deployment and on optimization of veto shield performance.

\section{C.1. Enhancement of the active shield}

The active shield allows us to utilize a large fraction of the central tracking calorimeter $(80 \%)$ as fiducial mass. Thus, our 1 kiloton detector has about the same fiducial mass as the 3-kiloton Kamiokande experiment. This technique does leave us vulnerabie to shieid inefficiencies and malfunctions, however, particularly because we must require coincident 
hits in the inner and outer layers of the shield to signal cosmic ray activity. For example, the $6 \%$ inefficiency of the present shield gives a $10 \%$ background to track-like neutrino events. As our statistics increase, it is possible that this inefficiency will make tighter containment cuts necessary, reducing our fiducial mass. The addition of a third shield layer would effectively eliminate this inefficiency and the associated backgrounds.

\section{The HPW proportional tube array}

The Tufts group has continued to work toward the utilization of the 660 proportional wire chambers which we acquired from the Harvard-Purdue-Wisconsin (HPW) proton decay experiment in 1987. The chambers are constructed from extruded aluminum tubes of rectangular cross section. As discussed in previous Progress Reports, it is possible to retrofit these HPW chambers and to read them out with our active shield electronics. Since May 1991 there have been two panels of HPW chambers (about $70 \mathrm{~m}^{2}$ ) which have operated continuously as part of the shield ceiling. The singles rates and hits-versus-time profiles are found to be as expected for properly operating chambers.

Last year we completed the fabrication of all electronics required to activate the remainder of the HPW array, which has twelve additional panels of chambers (each panel constitutes $42 \mathrm{~m}^{2}$ of tracking area). Unfortunately, HPW deployment at the mine has continued to be delayed, due to difficulties encountered with deployment of tracking calorimeter modules. It is possible that HPW deployment may resume - after a three year pause this coming October, once the mine crew has finished with the installation of the central tracking calorimeter.

By September 1992 Tufts researchers completed a report which documents all aspects of the HPW chambers which are relevant to successful retrofitting, deployment, and operation (Ref. 16). Included are descriptions of (1) the HPW array as acquired, (2) the electronics used to integrate the HPW signals into the Tufts digital readout scheme, (3) the fix devised to remedy a congenital gas leak in the HPW modules, (4) measurements of the performance of individual HPW modules in a cosmic ray test stand at Tufts, and (5) measurements of the performance of the two HPW panels currently operating in the Soudan mine. A useful finding that emerged from our cosmic ray test stand work is that a dramatic improvement in response time can be achieved in HPW chambers by changing the field-shaping potential from ground to -600 volts.

\section{The TASSO array}

An array of proportional tube modules which was used in the TASSO experiment at PETRA was shipped to the Soudan mine during summer 1991 and is currently being stored on Level 27. Static gas tests at Tufts showed that the chambers have pinhole leaks through the endplate at the electronics end, usually in the vicinity of the sense wire feedthrough. Fortunately these leaks - typically five per eight-cell chamber - can readily be sealed by epoxy. We concluded that, with non-skilled labor, the TASSO array can be made gastight to the level required of the veto shield. Last summer, the performance of TASSO chambers was investigated using our cosmic ray test stand at Tufts. Our conclusions are the following: The TASSO array amplifier/discriminator cards are in working order and are usable with the veto shield system. The chambers will require some modest tinkering to replace expired LEDs on the cards which are critical to the circuits' performance, to solder 
high voltage leads to the boards, and to set the circuit reference voltages to operating conditions optimized for a fill of the Soudan shield gas. Additionally, signal conversion boards will need to be built to convert the TASSO logic levels to those of the veto shield readout modules. The signal conversion is straightforward - a bit simpler even - than has already been implemented for the HPW readout. A detailed summary of our findings was written up as a "User's Guide to the TASSO Array" and was submitted to the Soudan collaboration in September 1992. ${ }^{17}$

\section{C.2. Soudan-II position survey}

For many of Soudan's physics analyses, e.g. contained events and multiple muons, it is necessary to know the relative positions of the central detector and of the veto shield. These positions are determined by measurements which are as direct as possible, but uncertainties are unavoidably introduced with measurements wherein physical obstacles have to be bypassed. A check is provided by a "muon survey", when throughgoing tracks reconstructed in the central detector are extrapolated to the veto shield and matched to shield hits. It turned out that there existed an inherent uncertainty in determining the relative vertical positions of the central detector and the veto shield, an uncertainty which did not occur with reconstruction in the shield by itself. The source of this uncertainty was finally eliminated during April when a new determination of the elevation of the central detector was made. Incorporating other recent measurements resulted in a shift in the vertical position of the veto shield by 3 to $10 \mathrm{~cm}$.

All additions to the veto shield which have been installed since summer 1992 have been surveyed, and best determinations of manifold positions have been entered into the database.

\section{D. Physics analysis at Tufts}

\section{D.1. Atmospheric neutrino flavor anomaly}

In Section 4.B.2 we described the novel interpretation of the atmospheric neutrino ratio anomaly proposed by Tufts researchers Mann, Kafka, and Leeson, which is that proton decay rather than neutrino oscillations is the origin of the anomaly. ${ }^{12,13}$ The authors are preparing to investigate new data from Soudan and from Kamiokande with the hope of distinguishing between the proton decay and $\nu$ oscillation hypotheses. A crucial prediction of the $\mathrm{p} \rightarrow \mathrm{e}^{+} \nu \nu$ hypothesis is that the anomalous $\nu_{\mu} / \nu_{e}$ ratio will revert to its expected value for $E_{\nu}$ above $600 \mathrm{MeV}$, in contrast to $\nu$ oscillations which imply that the ratio stays anomalous at higher energies. As of this date, the Kamiokande data are ambiguous on this point. In IMB-3 data, the ratio stays low for $300<E_{\nu}<1200 \mathrm{MeV}$ and therefore favors neutrino oscillations. However, the IMB-3 and Kamiokande data do not match up in a reasonable way in the $E_{\nu}>600 \mathrm{MeV}$ regime, and it is possible that there are pattern recognition problems with IMB-3 track versus shower separation at these energies (Ref. 18). Tufts researchers are carrying out a program intended to extract energctic event samples from Soudan data (see below), with the goal of resolving this crucial issue. 


\section{D.2. Partially contained neutrino interactions and upward-going muons}

We have developed software selection procedures which identify candidate neutrinoinduced interactions which are partially contained within the fiducial volume of the iron tracking calorimeter. The algorithm is essentially the following: We select events having localized hits which span the fiducial boundary used to define containment $(20 \mathrm{~cm}$ inside all outer surfaces of the calorimeter). We then eliminate events having coincident shield hit configurations characteristic of multiple muons or through-going single muons. Furthermore, we eliminate single track events which have been tagged by the SEARCH pattern recognition code as being downward-going, stopping muons. Finally, we eliminate all events which are coincident with double-layer hits in the ceiling panels of the shield. This latter cut is severe, and undoubtedly removes some genuine neutrino-induced events, but is necessary to reduce the candidate sample to a size which can be scanned by physicists.

Data runs from April 24, 1991 until July 7, 1992 were processed for these partially contained events (PCEs). The exposure corresponds to about 0.51 kiloton years; a total of 5439 candidate $\mathrm{PCEs}$ were selected and were subsequently scanned at Tufts using event displays provided by the collaboration's STING software. The near-final event samples were then submitted to physicists from Argonne and from Rutherford to obtain independent evaluations. The final event sample includes:

Multi-prong, partially contained neutrino induced interactions: 9 events

Events with upward energy flow:

12 events

The nine neutrino-induced multi-prong PCEs are on average more energetic than the collaboration's contained events and are spectacular in appearance; an illustrative event is shown in Fig. 4.2. The efficiencies of our procedures to find these events need to be carefully ascertained using Monte Carlo event samples. We note that our sample of nine events compares favorably with the number of contained multi-prong neutrino interactions which the collaboration has found in a similar exposure, using much different criteria.

Fig. 4.3 compares the visible final state energy in our PCE sample (Fig. 4.3c) with the visible energies from Soudan's contained quasi-elastic sample (Fig.4.3a) and contained multi-prong sample (Fig. 4.3b). The visible energy of the partially contained sample has a distribution which is similar to that of the contained multiprong events. Since the PCEs have additional energy which is not imaged (because tracks exit the detector), the sample represents neutrino interactions which are more energetic than those which compose Soudan's contained event sample. In order to extract a flavor ratio, a larger PCE sample is clearly needed. Processing and scanning are now underway at Tufts using data recorded from July 1992 to July 1993, and a kiloton-year PCE sample should be available by end of this summer.

In collecting events having an upward energy flow - the 12 event sample mentioned above - it was our intention to isolate a sample of upward-going, stopping muons which are initiated by high energy neutrino interactions in the rock below the detector. Among the 12 events, only 2 are compatible with the upward stopping muon interpretation. To our surprise, the remainder of the events are upward-going showers ( 3 events) and upward but nearly horizontal charged pions. A possibility is that the events are "splash-back" 
remnants of deep inelastic muon interactions within the rock around the detector. More study - and more statistics - are needed to clarify the origin of these events.

Investigation of partially contained events and of upward-traveling events are being carried out by Tufts graduate student William Leeson as part of his doctoral research.

\section{D.3. Underground multiple muon events}

We are investigating multiple muon events (muon bundles) recorded underground at a depth of 2090 mwe. To penetrate to this depth, the muons must have energies above $0.8 \mathrm{TeV}$ at the Earth's surface. The primary cosmic ray nuclei which give rise to the observed muon bundles have energies at incidence upon the upper atmosphere of 10 to $10^{5}$ $\mathrm{TeV}$. Muon bundles which have at least one muon traversing the tracking calorimeter are reconstructed using tracks in the calorimeter together with hit patterns in the surrounding $14 \mathrm{~m} \times 10 \mathrm{~m} \times 31 \mathrm{~m}$ proportional-tube shield. All ionizations are required to be coincident within 3 microseconds.

Two different data sets, one obtained July-August 1990 and a second set obtained during April through July 1991, have been used for the analysis of underground muon bundles. The latter sample, which contains about 55,000 multiple muon events, is the basis for the results presented here. An illustrative muon bundle event is shown in Fig. 4.4. In the Figure, the outer solid line represents the veto shield, and the smaller dotted outline depicts the central detector. Track 1 is detected within the central detector, with exit points shown by open circles. The full circles denote the veto shield exit points of the track extrapolated from the central detector. All other tracks are reconstructed using veto shield hits only.

In general, reconstruction of high multiplicity muon bundle events poses significant pattern recognition problems in Soudan II. The tracking calorimeter allows - in principle - all muons which traverse it to be clearly resolved. In practice, however, the multiplexing of readout electronics makes automated track reconstruction difficult and, in fact, high multiplicity events such as the 14 muon bundle shown in Fig. 4.5 have required human intervention in the pattern recognition. The veto shield array - because it is a simpler tracking system - has proven more amenable to computerized pattern recognition, although its coarse granularity $(20 \mathrm{~cm})$ currently limits the accuracy achievable with this approach. Difficulties inherent with reconstructing high multiplicity muon bundles with the shield array can be surmised from Fig. 4.6, which depicts the manifold hit configuration from a candidate 12-muon event. In analysis undertaken at Tufts, we have relied heavily on reconstruction algorithms which utilize coincident hits in the veto shield.

\section{D.4. Nuclear composition of high energy cosmic rays}

A goal of this study is to investigate the relative nuclear abundances in the primary cosmic radiation around the "knee" region $\left(10^{3}-10^{4} \mathrm{TeV}\right)$ of the incident energy spectrum. Four models for the nuclear composition of cosmic rays are considered: The Linsley model, the Constant Mass Composition model (CMC), the Maryland model, and the Proton-poor model (see Ref. 19). In order to compare our samples of reconstructed muon bundle 
events with expectations of the models, we rely on Monte Carlo simulations which impose all detector limitations and inefficiences onto events generated using the models.

The distribution of the number of muons $N_{\mu}$ within muon bundles can be used to study the primary composition of cosmic rays because the multiplicity depends on the number of nucleons $A$ of the primary particle. In principle, the observed muon multiplicity distribution can be compared with Monte Carlo expectations for a given nuclear composition and energy spectrum to determine the composition of the cosmic ray flux. Comparison of our observed multiplicity distribution with the multiplicity distributions obtained using the four different composition models is shown in Fig. 4.7a through 4.7d. The average multiplicity, $\left\langle N_{\mu}\right\rangle$, is $2.51 \pm 0.01$ for multiple muon events with two or more muons in a bundle.

For muons observed underground, the lateral distribution of muons about the core of the cosmic ray shower at any depth can be described by a form which falls off with increasing muon separation $r$ from the core. Unfortunately, uncertainty in the location of the shower core makes measurement of $r$-values difficult. For this reason we have analyzed our data using the inter-muon separation, $R_{i j}$, as measured in the plane transverse to the bundle direction. The distribution $\mathrm{d} N / \mathrm{d} R_{i j}$ is shown in Fig. 4.8 for the data and for two representative composition models. Spatial separations between muons within individual bundles of up to 30 meters are observed. The models yield distributions with shapes similar to but slightly narrower than the data. The average separation changes by only $6 \%$ between the extreme models which are the Linsley model (light composition) and the Maryland model (heavy composition). The average $R_{i j}$ for the Linsley model and the Maryland model are 6.9 and 7.1 meters respectively, to be compared with $7.1 \pm 0.3$ meters for the data sample.

A systematic search for possible correlations involving muon bundle multiplicity, intermuon separation within bundles, and bundle zenith angle has been carried out; comparable analyses from underground experiments have not appeared in the literature. Within a zenith angle range from $15^{\circ}$ to $60^{\circ}$, we observe the average muon multiplicity to remain constant, independent of zenith angle. The average inter-muon separation within a muon bundle, $\left\langle R_{i j}\right\rangle$, is observed to increase mildly with increasing zenith angle. Additionally, as shown in Fig. 4.9, there is an apparent increase of $\left\langle R_{i j}\right\rangle$ with increasing muon bundle multiplicity, for $N_{\mu}>8$. Neither of these mild correlations are included in the commonly used parameterizations of the nuclear composition models.

It is the lighter composition models, namely the CMC .nodel and the Linsley model, that show better overall agreement with our data, especially in the muon bundle multiplicity distribution and in the variation of average multiplicity with zenith angle. Independent fits to bundle distributions involving $N_{\mu},\left\langle N_{\mu}\right\rangle$ versus cosine of the zenith angle, and $\left\langle N_{\mu}\right\rangle$ versus $R_{i j}$, were carried out using a simplified two-component nuclear composition. The component distributions and the data are shown in Figs. 4.10, 4.11, and 4.12. Rough agreement is found among these fits; we infer that the primary cosmic radiation consists predominantly $(74 \pm 4 \%)$ of protons and relatively light nuclei (He, $\mathrm{CNO})$; elements heavier than $\mathrm{Mg}$ constitute the remaining fraction.

These results are presented in the doctoral dissertation of N. Sundaralingam of Tufts 
University. Follow-up studies, using a five-fold increase in statistics and a new muon reconstruction algorithm, are underway by doctoral student Bruce Ewen.

\section{E. Additional physics results from Soudan-II}

\section{E.1. Search for GUT magnetic monopoles}

In the Soudan-II detector, a through-going GUT magnetic monopole would give rise to a long straight track having ionization significantly higher than the average muon. The ionization would be uniformly heavy along the entire track, but knock-on electrons would be completely absent. A sample of tracks having reconstructed images longer than $1.5 \mathrm{~m}$ and having a pulse height per unit length of at least four times the average for cosmic ray muons, was collected over a period of about three years with a total exposure of $2.64 \times 10^{14}$ $\mathrm{cm}^{2}$ sr s. The search was sensitive to GUT monopoles having $\beta>2 \times 10^{-3}$.

A total of 10,254 events were flagged by the software as possible monopole candidates. For each of these, event displays of the raw data were scanned by a physicist. The main criteria for rejection of events during the scan were based on the fact that a monopole of GUT mass would be uniformly heavily ionizing and could not make a visible knock-on electron or a larger electromagnetic shower. That the presence of a knock-on electron can be used to unambiguously reject the monopole hypothesis, is particularly effective in Soudan-II at the lower monopole ionizations (slow velocities), where a muon and a monopole might be confused. This point is illustrated by the through-going muon image shown in Fig. 4.13. As a result, we are able to assign a limit on the monopole flux (90\% CL), of $8.7 \times 10^{-15} \mathrm{~cm}^{-2} \mathrm{sr}^{-1}$ for $\beta$ greater than $2 \times 10^{-3}$. This study was published in Physical Review D in December 1992. ${ }^{20}$

\section{E.2. All-sky search for astrophysical sources using underground muons}

In 1991, the Soudan-II collaboration reported evidence for underground muons associated with an intense radio flare emitted by the binary star system Cygnus $\mathrm{X}-3,{ }^{21}$ a candidate cosmic ray point source which is in our galaxy. The highest energy cosmic rays are expected to be extra-galactic in origin. The EGRET group ${ }^{22}$ has published a preliminary catalogue of high energy $(<20 \mathrm{GeV})$ gamma-ray point sources. The locations in equatorial coordinates for gamma ray sources which are visible from the Soudan site is indicated in Fig. 4.14. This catalogue includes active galactic nuclei (AGN) 3C273 and Markarian 421. Recently, the Whipple group ${ }^{23}$ has published evidence for a point source of $\mathrm{TeV}$ photons associated with Markarian 421.

The large acceptance of the Soudan-II detector allows a wide search for point sources of underground muons to be made. Muons recorded over a period of 49 months in the SoudanII underground detector have been used to examine the entire sky within the acceptance of the detector (declination, $\delta$, between $-15^{\circ}$ and $+85^{\circ}$, and all right ascensions, $0<\alpha<360^{\circ}$ ) for evidence of steady point sources of cosmic rays. Two 2-D histograms (maps) of the celestial sphere for declinations greater than $-15^{\circ}$ were made. The first map contained the 14 million muons in the data set binned in right ascension $\times$ declination pixels of $0.1^{\circ} \times 0.1^{\circ}$. The second map contained the expected number of background events in each pixel. The background was estimated by obtaining the hour-angle distribution of the data 
for each $0.1^{\circ}$ declination band and then convoluting this with the sidereal time distribution of the data, obtained from the arrival time of each muon in the data set. In the absence of correlations between the observed hour angle of a muon and the sidereal time of its arrival (i.e. point sources), this procedure predicts the number of events expected in each pixel due to the local isotropic background and necessarily constrains the total number of background events to be equal to the total number of events observed.

Since the angular resolution of the detector is $1.0^{\circ}$, a $1.6^{\circ}$ half angle cone was chosen for the point source search. The numbers of events observed and expected in a cone of $1.6^{\circ}$ space angle centered on each pixel of the map were then counted by summing the contents of all pixels within $1.6^{\circ}$ of the cone center. The background and data maps were treated identically and a map of deviations with 3.6 million $0.1^{\circ} \times 0.1^{\circ}$ pixels was constructed, as shown in Fig. 4.15. The contents of the pixels of this map are correlated unless they are separated by a space angle of at least $1.6^{\circ}$.

The deviations for all cone centers for which the expected number of background events was greater than 200 were examined. No unambiguous evidence was found for any point source of underground muons. The maximum positive excess was found to be 4.87 standard deviations for a cone centered at $\alpha=189.55^{\circ}, \delta=1.55^{\circ}$ (bin center). Fig. 4.16 shows a contour map, in deviations, of $\mathrm{a} \pm 10^{\circ}$ area around this point. A clear peak of $3^{\circ}$ half-width in declination but somewhat elongated in right ascension is visible. The greatest deviation is $5.13 \sigma$ at these coordinates. The numbers of events expected and observed in a $1.6^{\circ}$ cone centered on this position are 2150 and 2388 respectively. The minimum zenith angle of a source at this position observed by the detector is $46^{\circ}$ corresponding to a minimum overburden of $3010 \mathrm{mwe}$. The excess events correspond to a flux of $8.3 \pm 1.7 \times 10^{-12} \mathrm{~cm}^{-2} \mathrm{~s}^{-1}$ assuming the same depth dependence as the background.

The galactic coordinates of the excess events are longitude $=296.8^{\circ}$ and latitude $=64.0^{\circ}$. There are no known nearby candidate sources at this location so the EGRET catalogue of AGN gamma-ray sources has been searched for a positional coincidence. The closest source is the powerful quasar $3 \mathrm{C} 273$, with celestial coordinates $\alpha=187.4^{\circ}, \delta=2.1^{\circ}$. The space angle between $3 \mathrm{C} 273$ and the excess bin center is $2.2^{\circ}$. The probability that a direction chosen at random within the acceptance of the Soudan II detector is within $2.2^{\circ}$ of any one of the EGRET sources is about $1 \%$.

A paper reporting the above observations is being prepared by the Soudan collaboration.

\section{E.3. Surface-underground coincidence measurements of cosmic ray showers}

A $41-\mathrm{m}^{2}$ detector of proportional tubes situated on the Earth's surface 714 meters above Soudan II at a zenith angle of $13^{\circ}$, has been operated in coincidence with the underground detector. When a high energy cosmic ray produces charged particles in both the surface and underground detectors, the underground muons are used to define the position and direction of the core of the extended air shower above the ground. The reconstructed shower size, the apparent number of charged particles in the shower as it passes the surface of the Earth, is a measure of the primary-cosmic-ray energy, and it is estimated from the shower density in the surface detector and its distance from the shower core. 
Data collected during 7,141 live hours between June 1991 and November 1992 has been analyzed by the Minnesota group. The surface detector required at least two shower particles within a 3 microsecond coincident window corresponding to an underground muon event. The underground muon multiplicity and the surface-detector multiplicity were restricted to $N_{\mu}<5$ and $N_{s u r f}<20$, respectively, and underground muon tracks longer than $1 \mathrm{~m}$ were required. In addition, events for which the surface detector-to-shower core separation was greater than $300 \mathrm{~m}$ were rejected. While this limits the energy range of the experiment (events with the shower core far from the surface detector are high in energy), it reduces a potential systematic error in shower size reconstruction due to differences between the predicted and observed particle distribution at large radii.

The all-particle flux is uncertain to about $30 \%$ in the range $10-10^{4} \mathrm{TeV}$. Nevertheless, it is useful to compare the data and Monte Carlo by absolute event rate. This is done by calculating the ratio of observed to predicted events as a function of reconstructed shower size. This ratio is the factor by which the Monte Carlo flux must be multiplied to match observations. In Fig. 4.17, the observed flux is compared to the four cosmic ray nuclear composition models. It is seen that only the Linsley model - a "light" composition model reproduces the observed event rate at large shower size. If a factor of two is considered the upper limit by which the Monte Carlo flux normalization could legitimately be in error, the P-poor model fails for $S>10^{4.4}$, corresponding roughly to a $10^{2.4}-\mathrm{TeV}$ proton primary. The Maryland model fails for $S>10^{5.1}\left(10^{3}-\mathrm{TeV}\right.$ proton), and the CMC model fails for $S>10^{5.8}\left(10^{3.6}-\mathrm{TeV}\right.$ proton).

Analysis indicates that the shower size reconstruction is statistically accurate, however there may be a systematic error in the Monte Carlo shower model. Such an error would have to be large - greater than a factor of two - in order to reverse the trends seen in Fig. 4.17. These findings have been prepared for presentation at the 23rd International Conference on Cosmic Rays, to be held in Calgary, Canada during July. ${ }^{24}$

\section{E.4. Energetic horizontal muons as evidence for AGN neutrinos}

A specific model for particle production in Active Galactic Nuclei leads to the possibility that neutrinos from AGNs may outnumber the atmospheric neutrino flux above an energy of $30 \mathrm{TeV}{ }^{25}$ The diffuse flux from all AGNs might be detectable even if single AGN sources cannot be identified. Muons initiated by interactions of high energy neutrinos underground have a large range in rock. For horizontal muons which undergo a large radiative energy loss in the Soudan-II detector, the background from cosmic ray muons will be relatively small. A high energy muon may deposit a large amount of energy in traversing Soudan-II as a result of electron pair production or violent bremsstrahlung, or it may only leave ionization. We calculate that $84 \%$ of $100 \mathrm{TeV}$ muons, $39 \%$ of $20 \mathrm{TeV}$ muons, and $10 \%$ of $5 \mathrm{TeV}$ muons will leave $40 \mathrm{GeV}$ or more of radiative energy loss in traversing Soudan-II. As we reported last year, we obtained limits for the flux of 5-, 20and $100-\mathrm{TeV}$ muons from diffuse sources to be 100,30 , and $10 \times 10^{-14} \mathrm{~cm}^{-2} \mathrm{~s}^{-1} \mathrm{sr}^{-1} \mathrm{re}$ spectively, which are comparable to the muon flux expected from the neutrino prediction of Stecker et al. ${ }^{25}$ Analysis of a larger data sample, and calculations of backgrounds for this method are under way. 


\section{Section 4 References.}

1. R. Becker-Szendy et al., Phys. Rev. D 47, 4203 (1993).

2. R. Becker-Szendy et al., "Recent limits on baryon non- conservation from the IMB3 experiment", submitted to the Proceedings of the 23rd International Cosmic Ray Conference, Calgary, July 1993.

3. H. Meyer, "Contained events from Frejus", talk presented at the Atmospheric Neutrino Workshop, LSU-HEPA-93-6, Louisiana State University, May 6-8, 1993.

4. S. Ahlen et al., Phys. Rev. D 46, 895 (1992).

5. A. Bettini, "The ICARUS experiment: status and program", in: Proceedings of the XXVI Int. Conf on HEP, Dallas, Texas, August 1992, Vol. II, p. 1657.

6. D.H. Perkins, "The atmospheric flux problem: a critique", Oxford Univ. preprint OUNP-93-03.

7. T.K. Gaisser, in: Proc. Workshop on Long-baseline Neutrino Oscillations, Fermilab, November 1991. Published by Fermilab, Batavia IL, 1992, p.111.

8. K.S. Hirata et al., Phys. Lett. B 280, 146 (1992).

9. T. Kajita (Kamiokande Collaboration), talk presented at the International Symposium on Neutrino Astrophysics, Takayama/ Kamioka, Japan, 19-22 Oct. 1992.

10. D. Casper et al., Phys. Rev. Lett. 66, 2561 (1991).

11. R. Becker-Szendy et al., Phy. Rev. D 46, 3720 (1992).

12. W.A. Mann, T. Kafka, and W. Leeson, Phys. Lett. B 291, 200 (1992).

13. W.A. Mann, T. Kafka, and W. Leeson, in: Proceedings of the DPF92 Meeting, Fermilab, 10 - 14 November 1992; p. 1330.

14. T.K. Gaisser, T. Stanev, G. Barr, Phys. Rev. D 38, 85 (1988); G. Barr, T.K. Gaisser, T. Stanev, Phys. Rev. D 39, 3532 (1989).

15. E.V. Bugaev and V.A. Naumov, Phys. Lett. B 232, 391 (1989).

16. B. Ewen, W.A. Mann, W.P. Oliver, and N. Sundaralingam, "The HPW enhancement of the active shield", Soudan-II note PDK-525, Sept. 1992.

17. K. Karr, M. Pfenning, W.A. Mann, W. Oliver, Y. Gopika, and T. Kafka, with authors from Oxford Univ., Soudan-II note PDK-524, Sept. 1992.

18. T. Mann, "Atmospheric neutrino anomaly as proton decay", talk presented at the Atmospheric Neutrino Workshop, LSU-HEPA-93-6, Louisiana State University, May 6-8, 1993.

19. C. Forti et al., Phys. Rev. D 42, 3668 (1990).

20. J. Thron et al., Phys. Rev. D 46, 4846 (1992).

21. M.A. Thompson et al., Phys. Lett. B 269, 220 (1991).

22. C.E. Fichtel, Preliminary EGRET Source Catalog, NASA preprint 93-14 (1993).

23. M. Punch et al., Nature 358, 477 (1992).

24. N. Longley et al., "Ultra high energy cosmic ray composition from simultaneous surface and underground measurements at Soudan 2", submitted to the Proceedings of the 23rd Int. Cosmic Ray Conference, Calgary, July 1993.

25. F.W. Stecker et al., Phys. Rev. Lett. 66, 2697 (1991). 


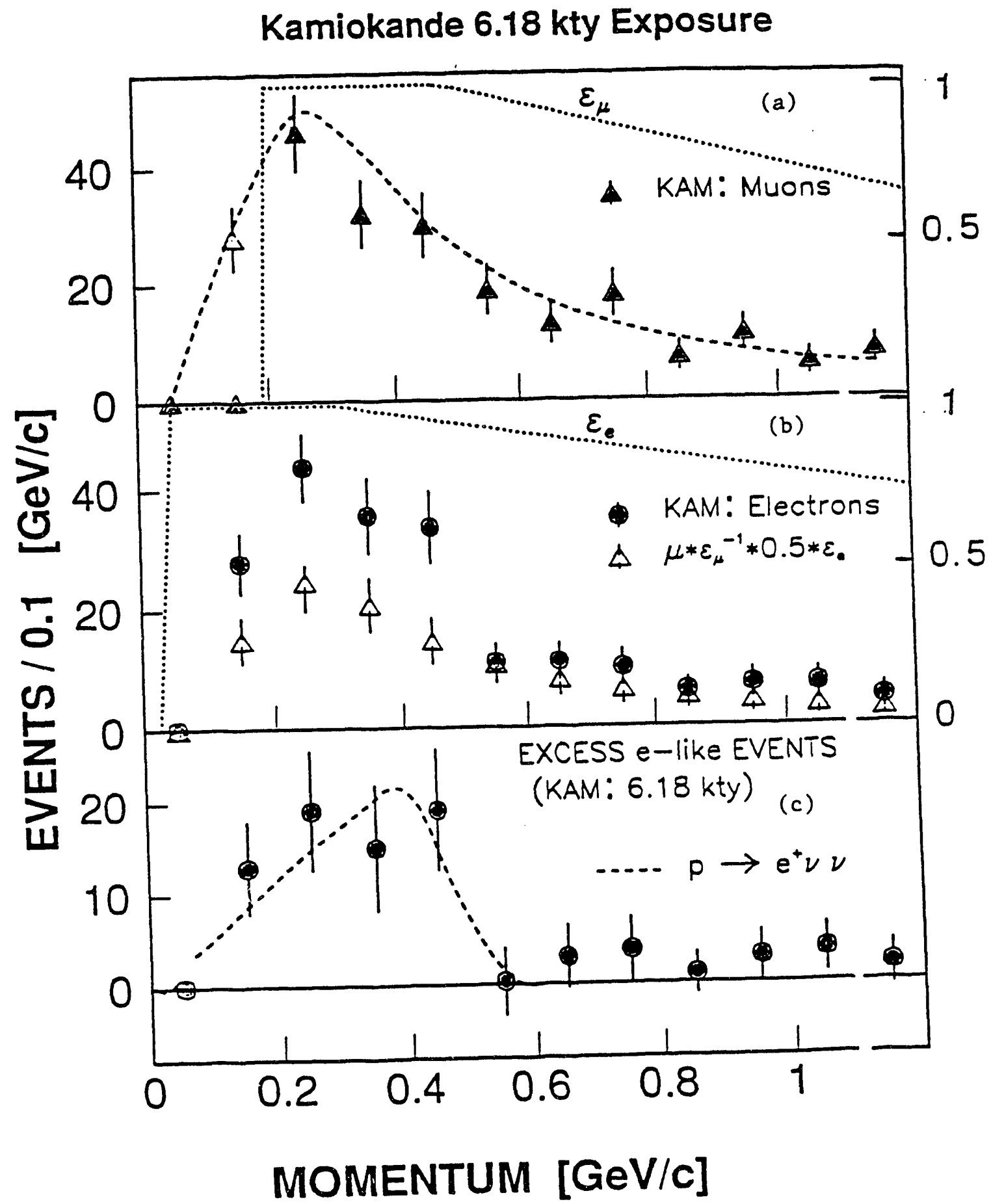

FIG. 4.1

Momentum distributions of single-ring (a) $\mu$-like and (b) e-like events from the $0.118 \mathrm{kty}$ exposure of Kamiobande. Detection efficiencies are shown superimposed (dotted). The dashed curve in (a) depicts the distribution shape expected for quasielastic events. It is used to infer the event rate in the lowest momentum bin (open triangle). 

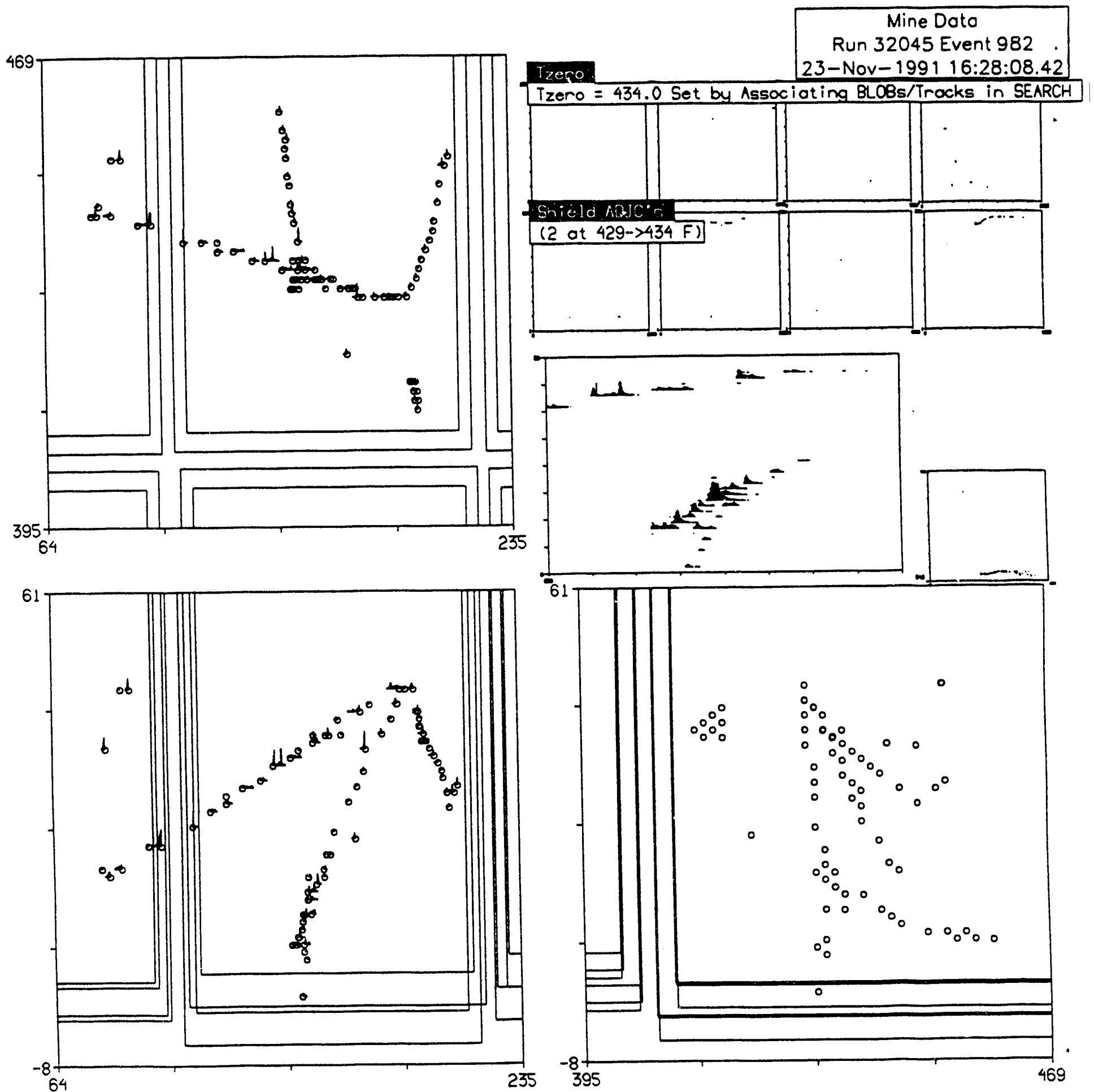

FIG. 4.2 


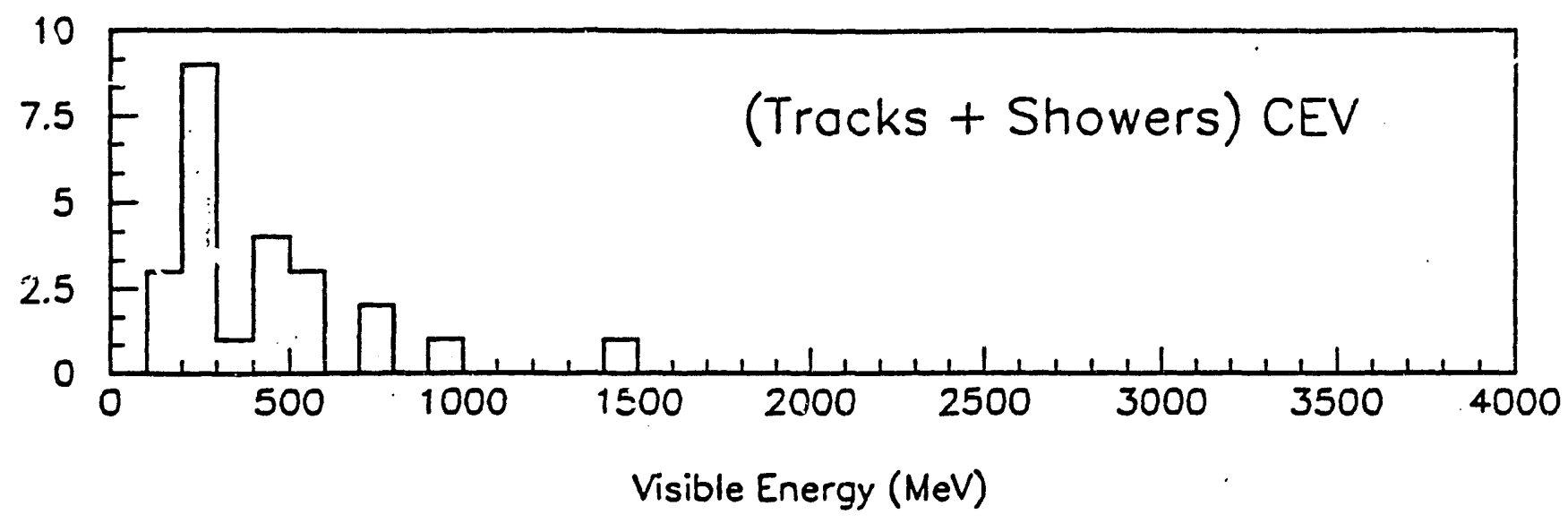

FIG. $4.3 a$


FIG. $4.3 c$

Visible Energy (MeV) 


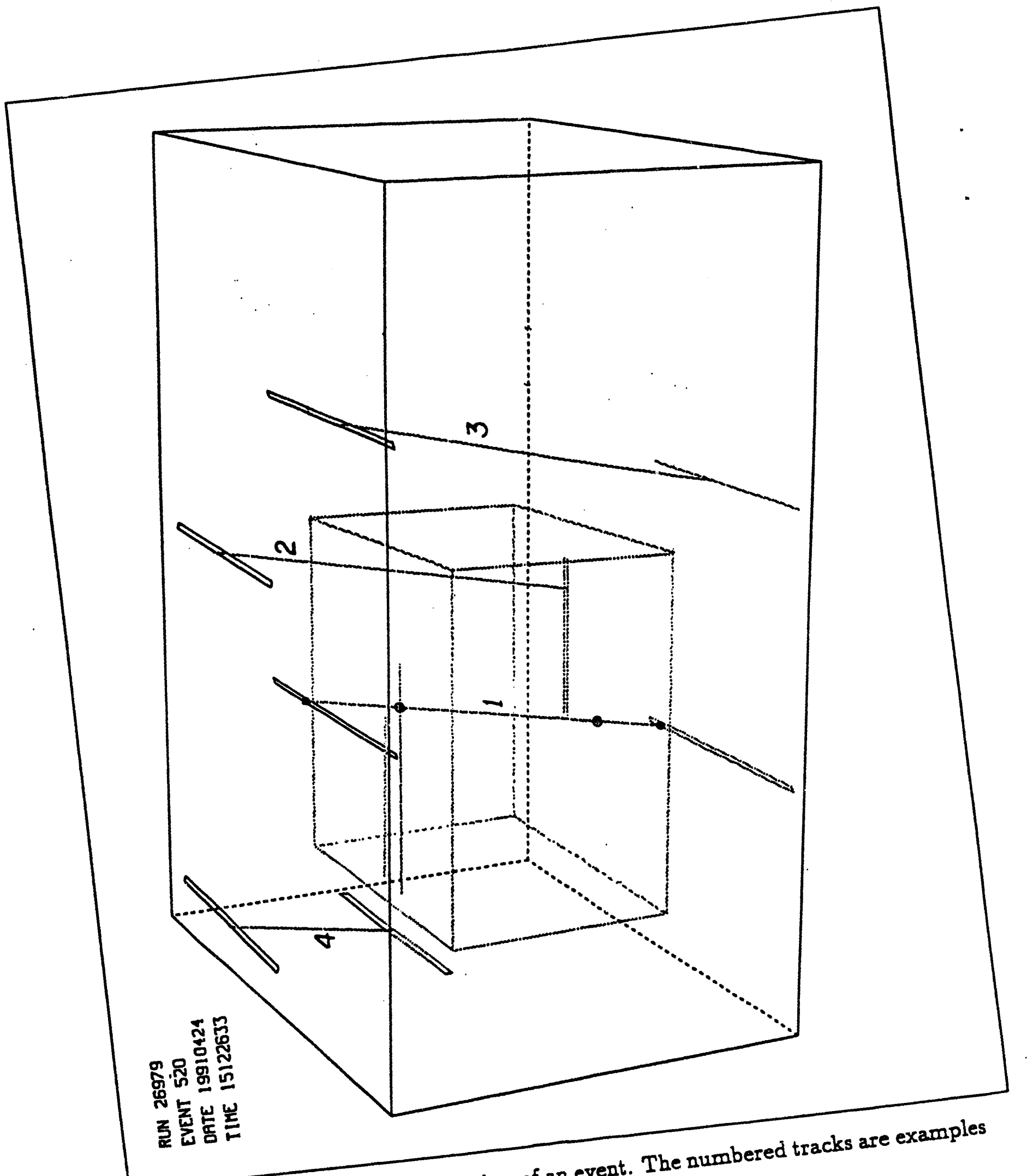

Three dimensional view of an event. The $n$ of the various classifications of candidate muon tracks.

FIG. 4.4 

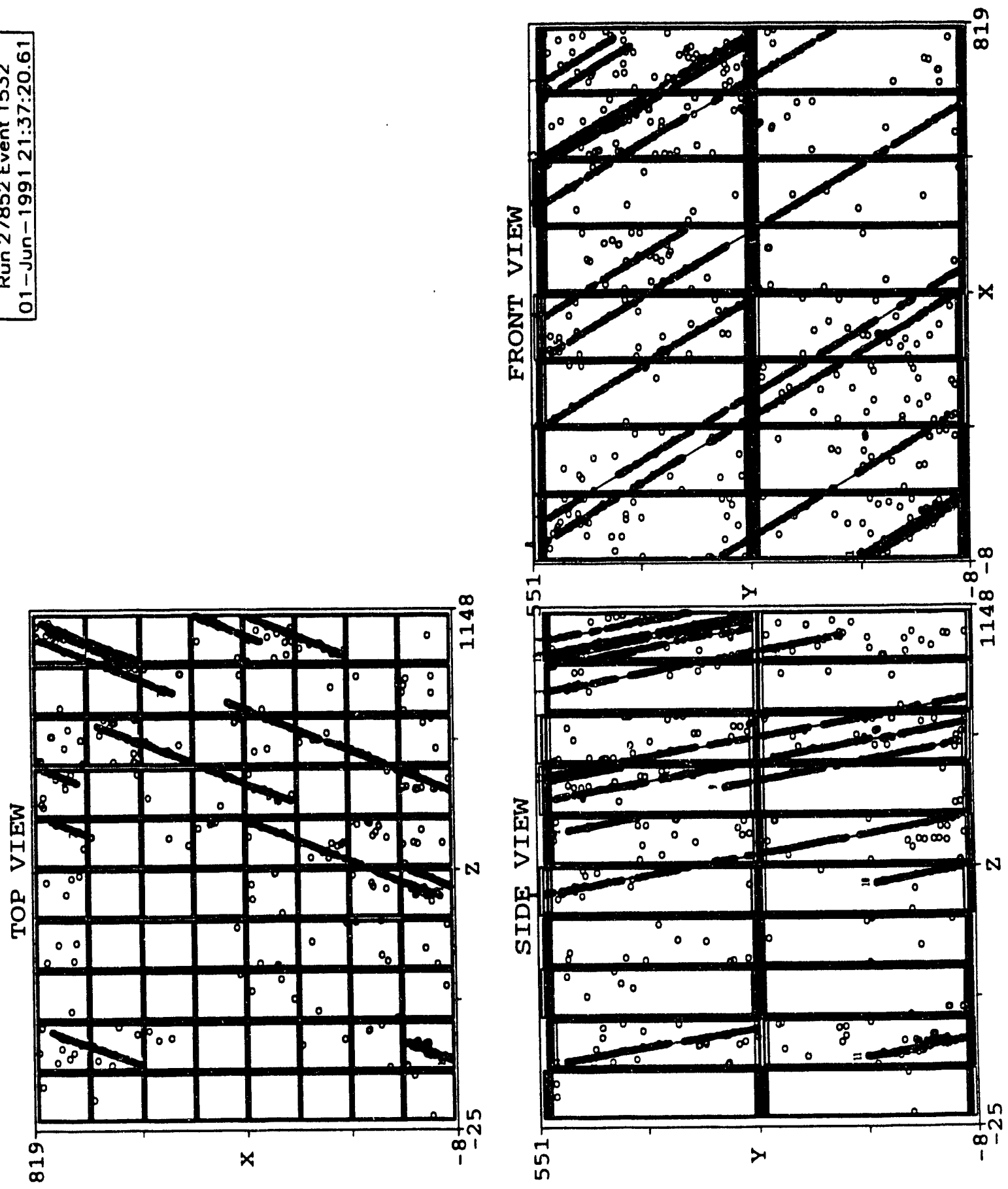

STING image of an event which has fourteen muons passing through the central detector.

FIG. 4.5 


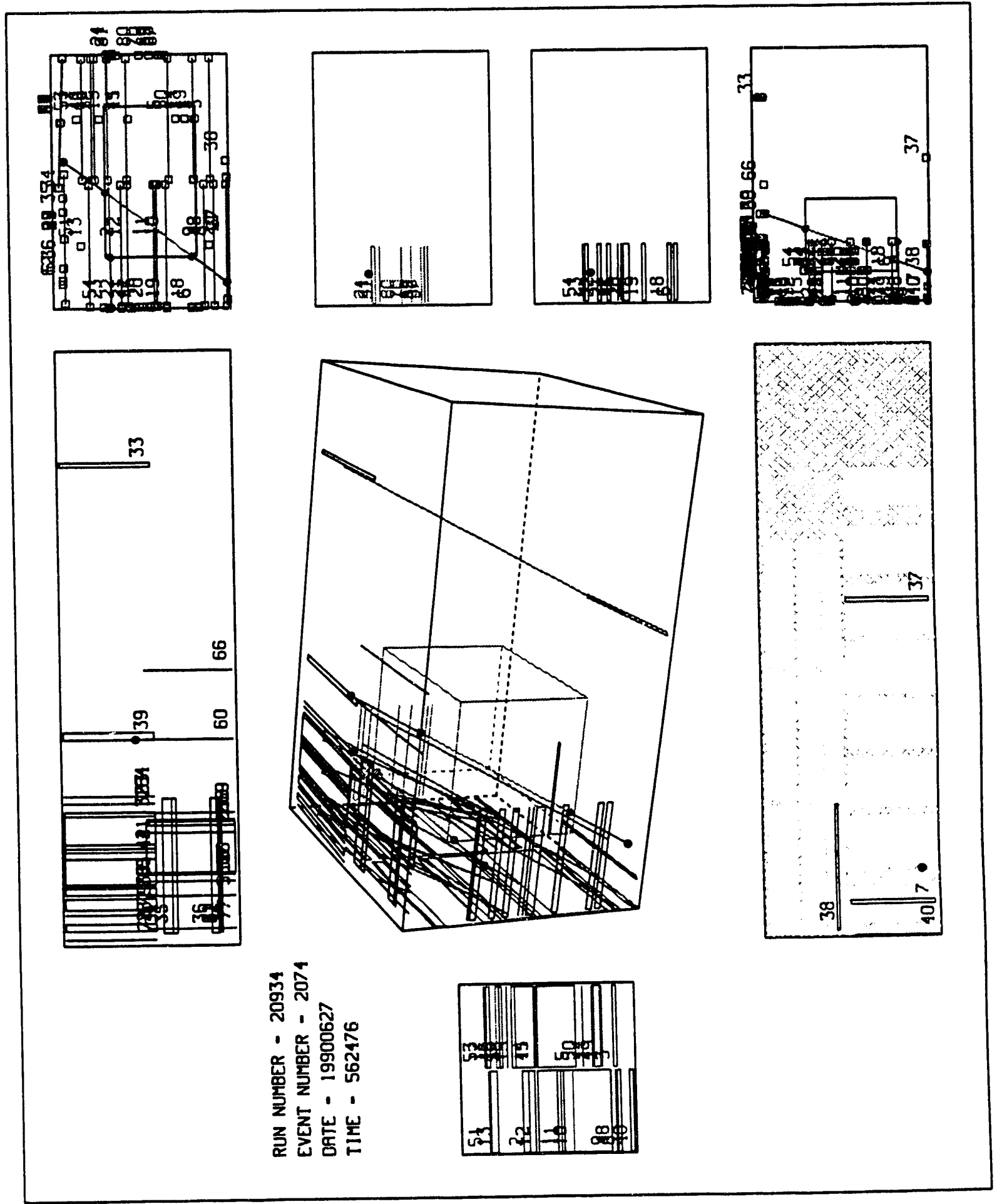

An Event Display showing the coincident hit patterns recorded on all veto shield surfaces in a twelve multiple muon event.

FIG. 4.6 


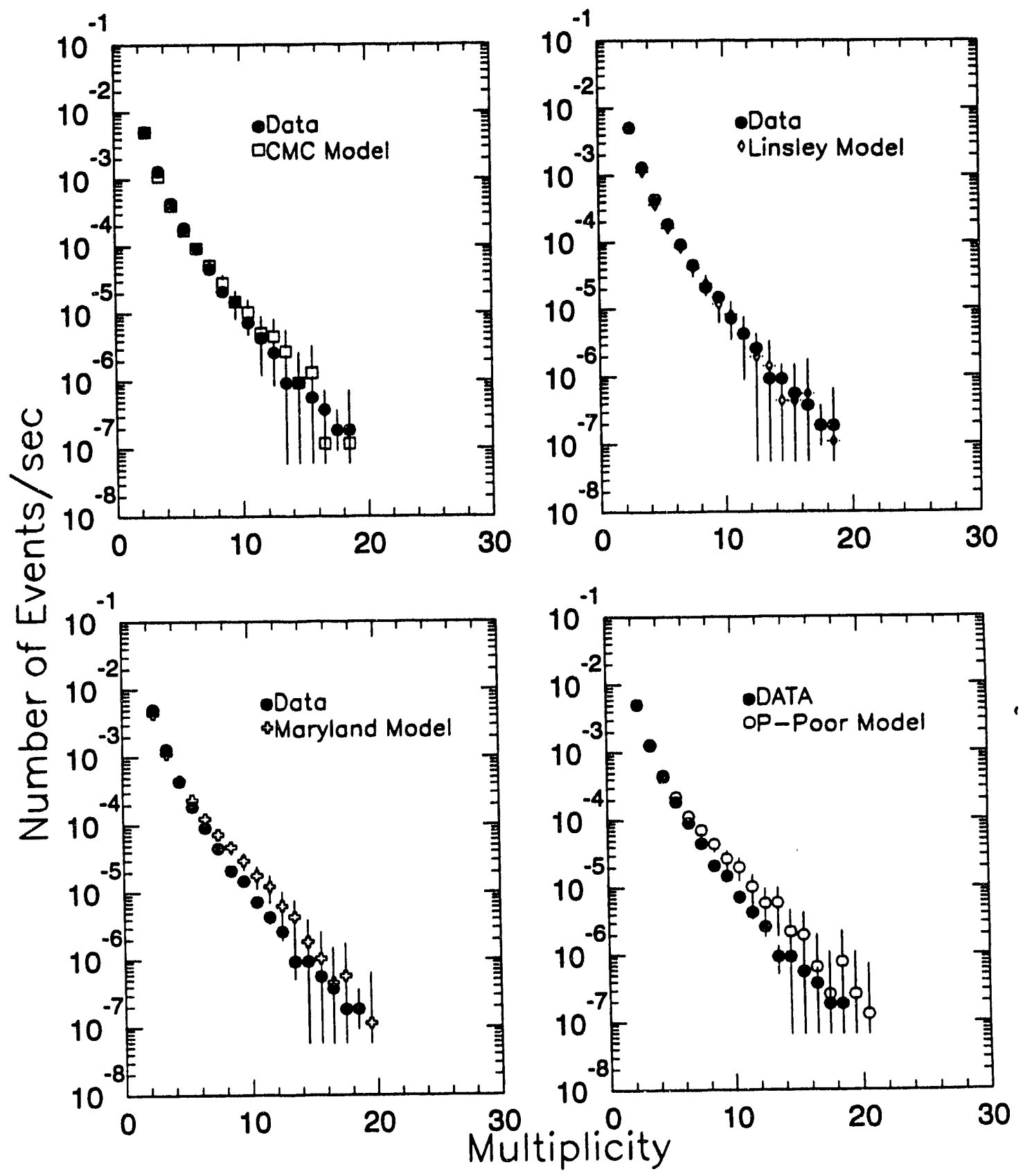

Comparison of muon bundle rates as a function of multiplicity, for the four composition models.

FIG. 4.7 


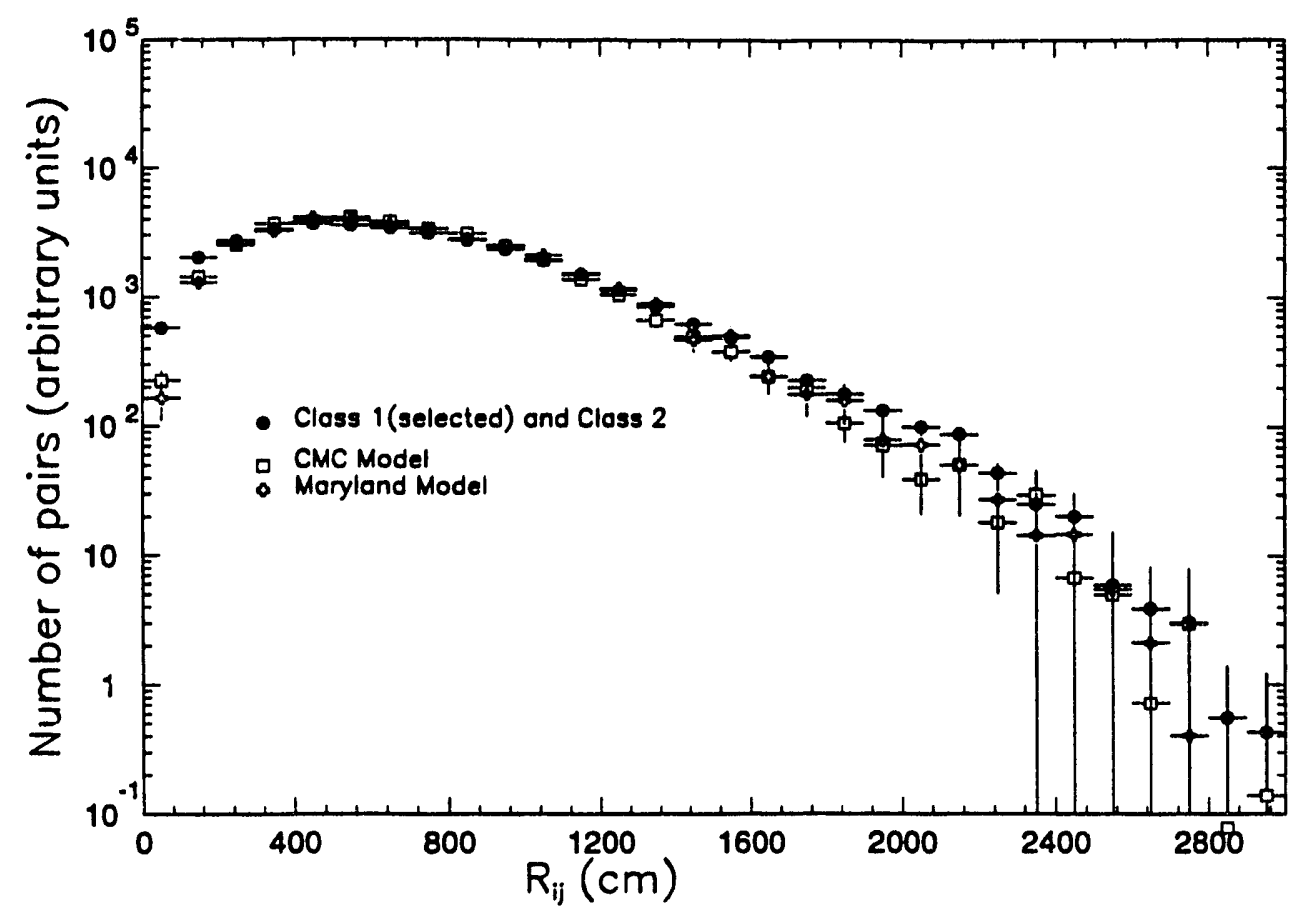

the models.

Distribution of separations between pairs of muons, in the data and in

FIG. 4.8

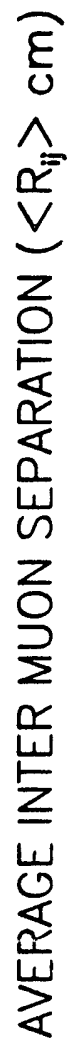

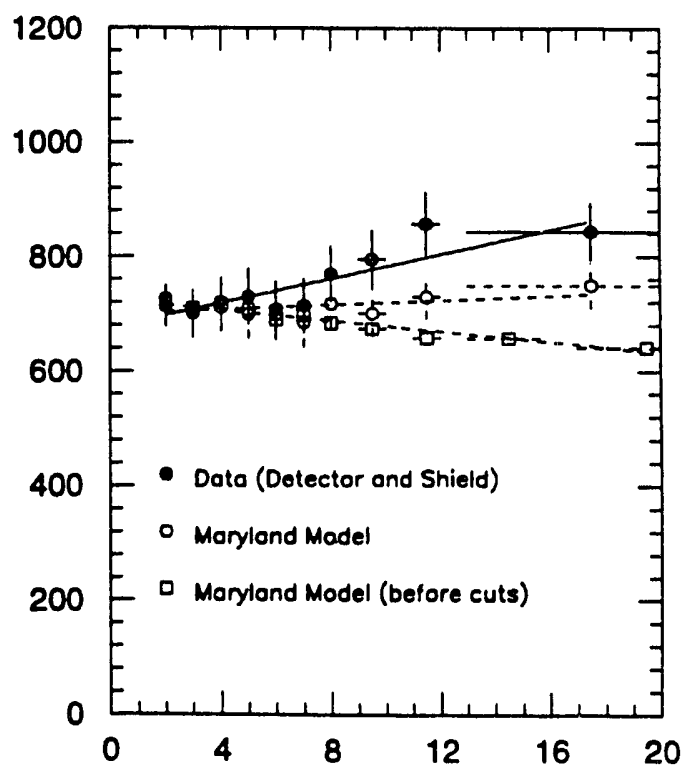

Intermuon separation versus bundle multiplicity 


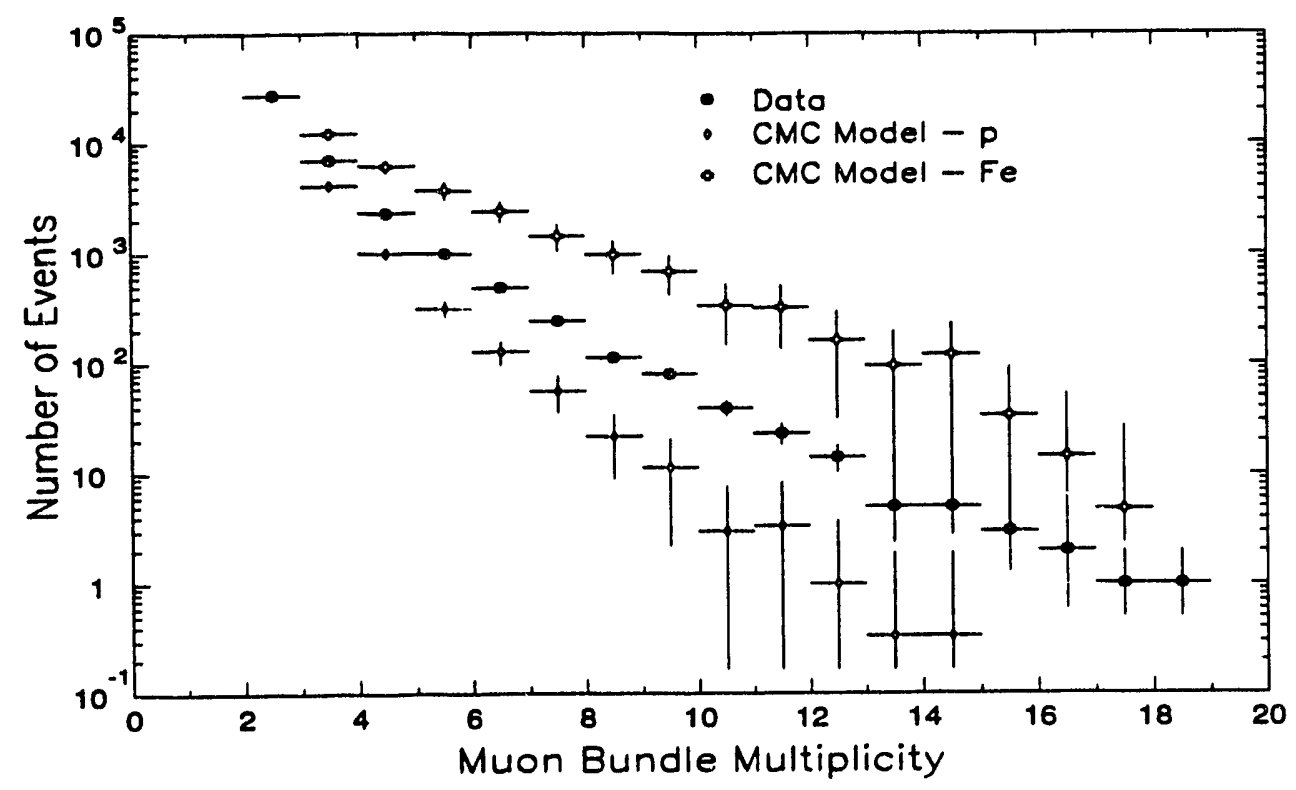

FIG. 4.10 Multiplicity distributions for data and for the CMC model with $p$ and Fe primaries only (normalized to the number of two muon events in the data sample).
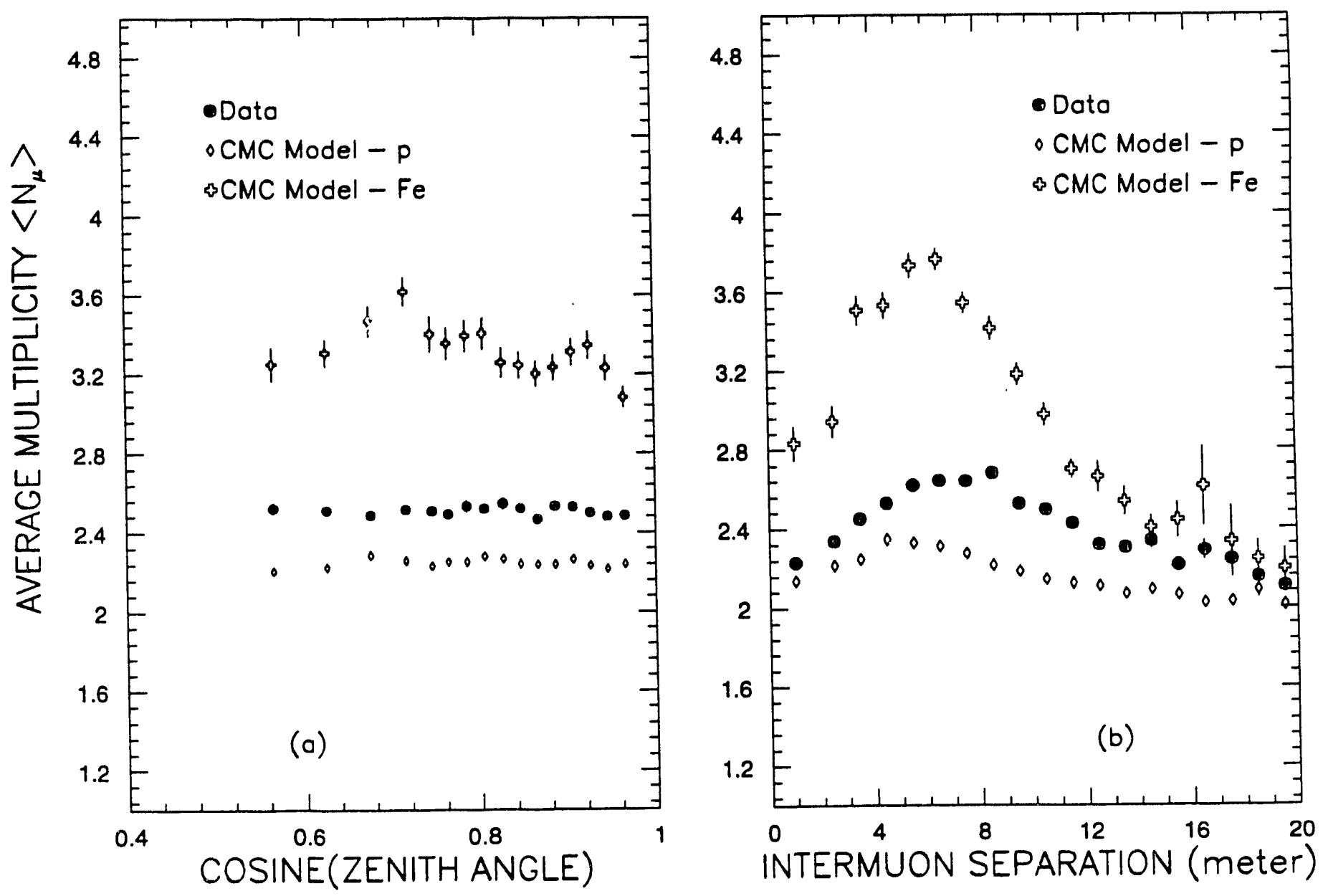

FIG. 4.11 


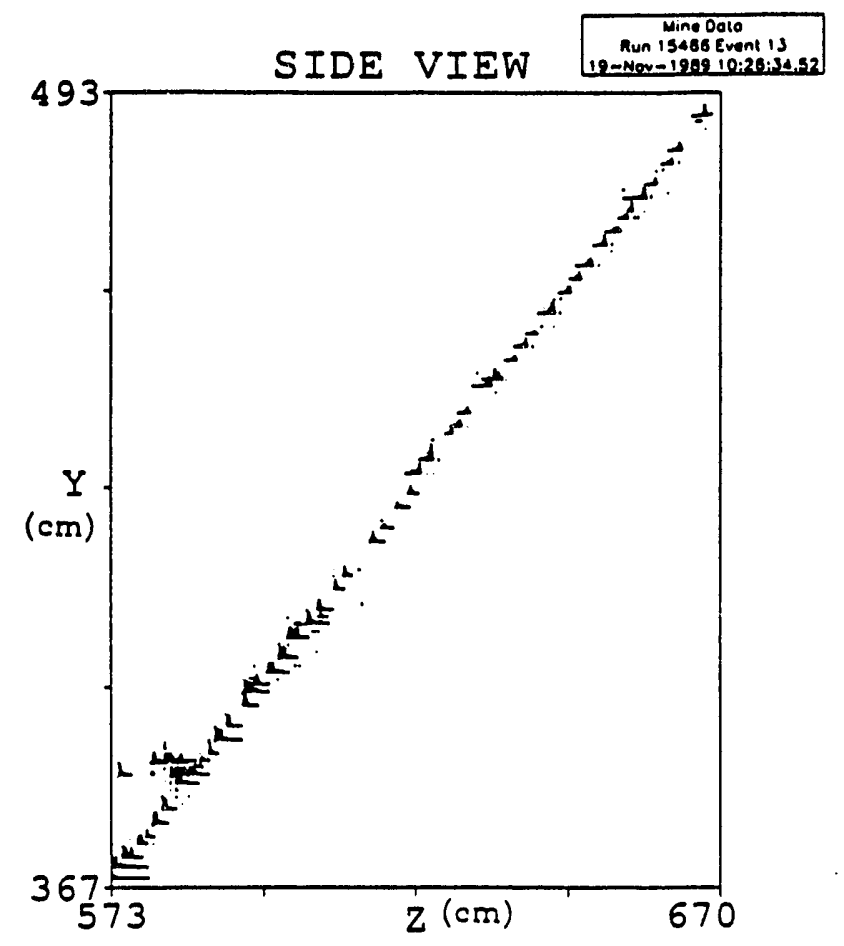

FIG. 4.13

An event with a small knock-on electron associated with the track. This muon track also failed the high ionization cut.

\section{Equatorial Coordinates}




Equatorial Coordinates

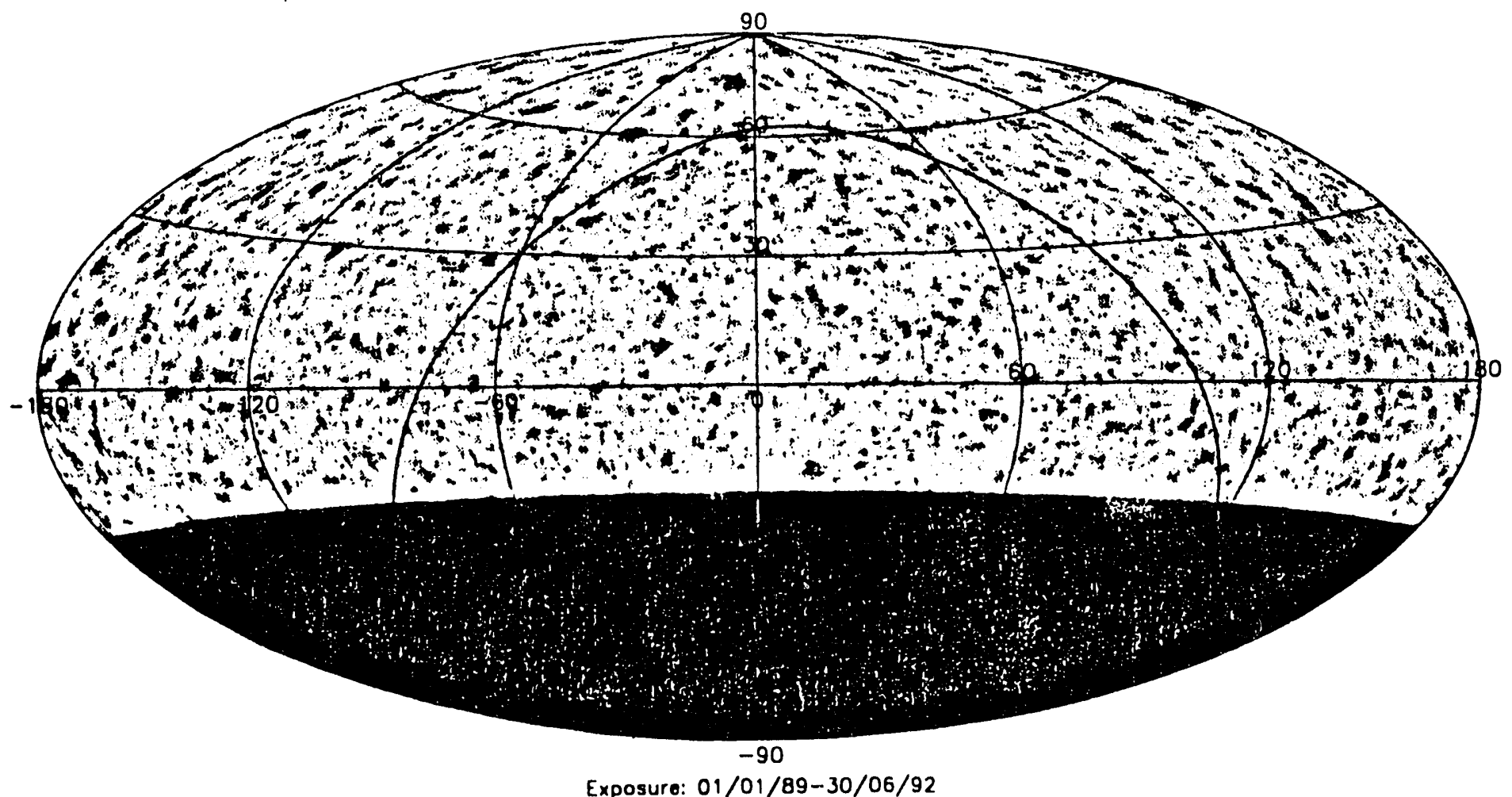

FIG. 4.15

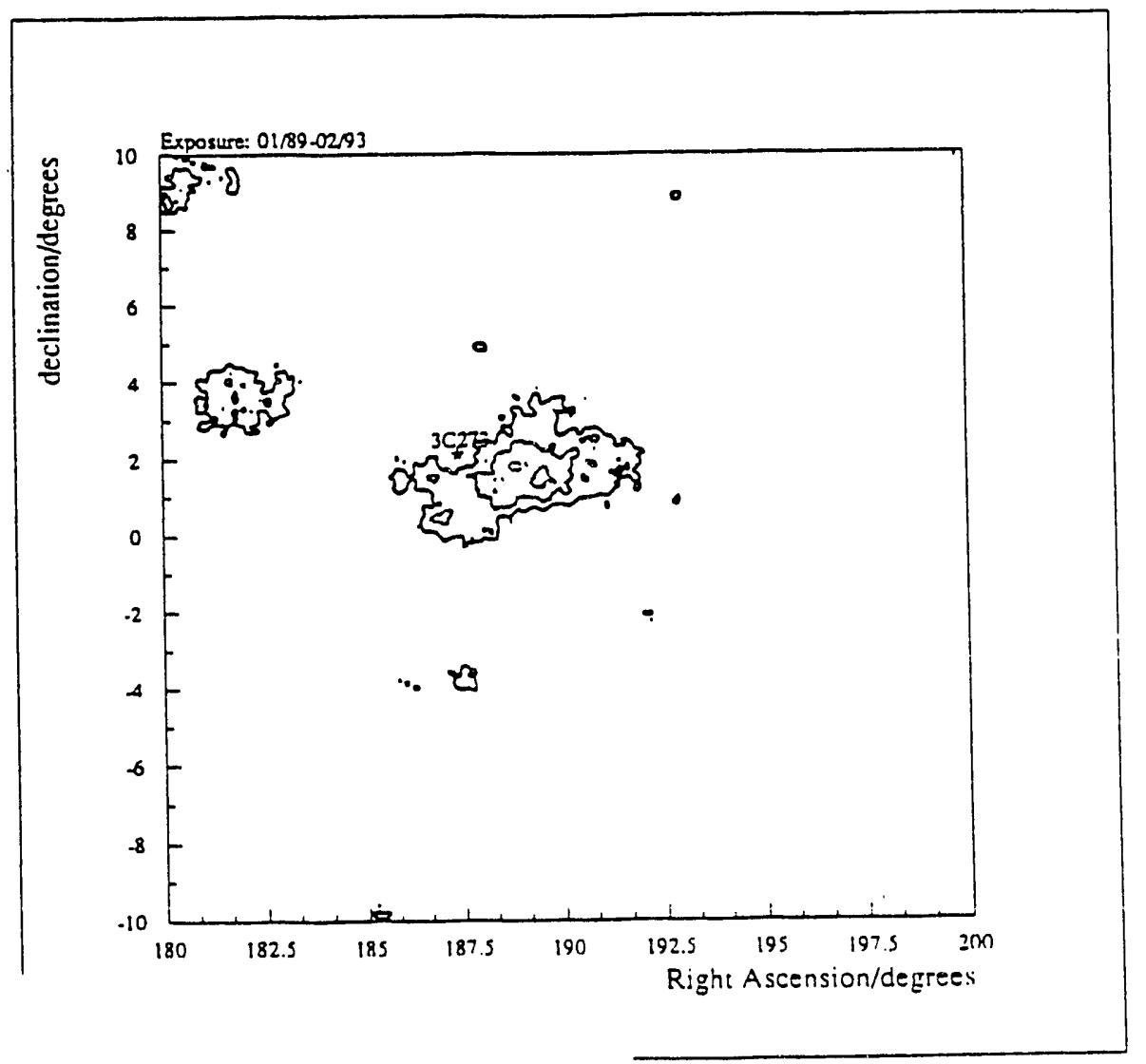




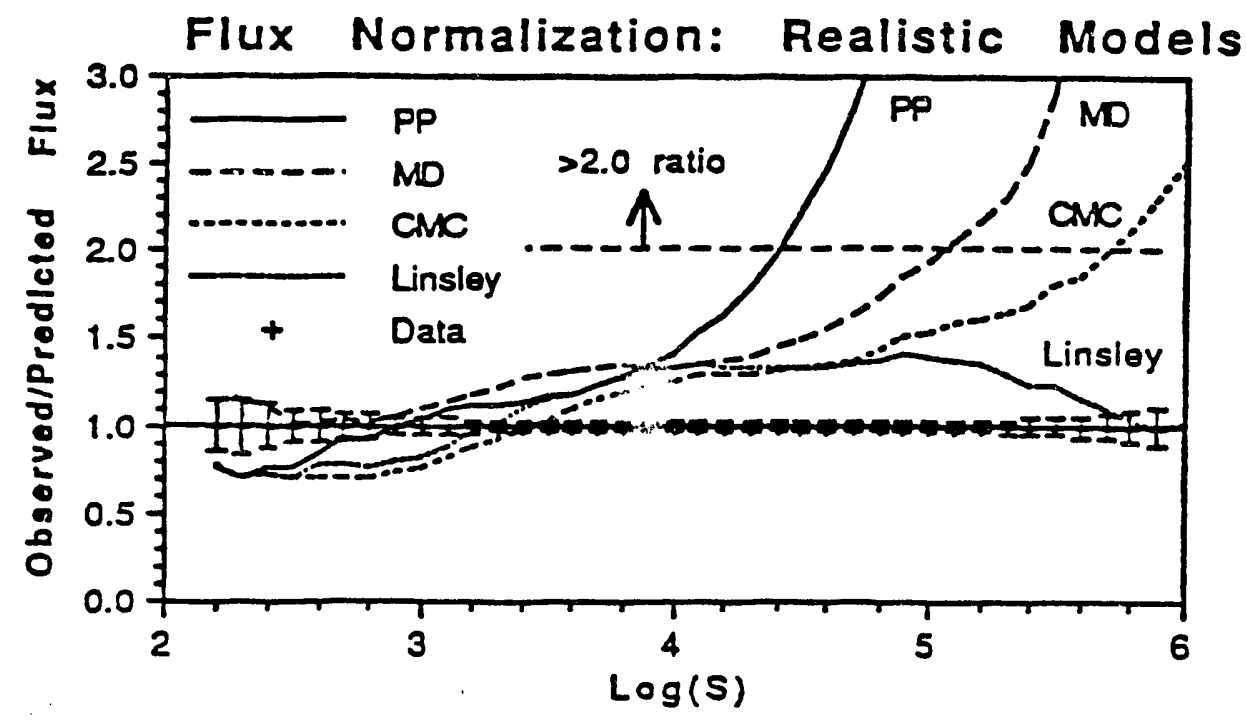

FIG. 4.17 


\section{Physics at the proton-antiproton collider at $\sqrt{s}=1.8 \mathrm{TeV}$}

(Collider Detector at Fermilab - CDF/E-741/E-775 - Prof. K. Sliwa, Post-Doctoral Research Associate M. Timko, Graduate Research Assistant M. Roach-Bellino, Graduate Research Assistant D. Benjamin, Graduate Student K. Karr - in collaboration with Argonne National Laboratory; Brandeis University; Istituto Nazionale di Fisica Nucleare (INFN), University of Bologna, Bologna, Italy; University of California at Los Angeles; University of Chicago; Duke University; Fermi National Accelerator Laboratory; Laboratori Nazionali di Frascati, Istituto Nazionale di Fisica Nucleare (INFN), Frascati,Italy; Harvard University; University of Illinois; Institute of Particle Physics, McGill University and Univerity of Toronto, Canada; National Laboratory for High Energy Physics, KEK, Japan; Lawrence Berkeley Laboratory; Massachusetts Institute of Technology; University of Michigan; Michigan State University; University of New Mexico; Osaka City University, Japan; Universita di Padova, Italy; University of Pennsylvania; INFN - University and Scuola Normale Superiore of Pisa, Italy; Purdue University; University of Rochester; Rockefeller University; Rutgers University; SSC Laboratory; Texas A\&M University; University of Tsukuba, Japan; University of Wisconsin; Yale University)

\subsection{Introduction}

The Standard Model (SM) of strong and electroweak interactions is based on SU(3) $C$ $\times \mathrm{SU}(2)_{L} \times \mathrm{U}(1)$ local gauge symmetry. It is consistent with all present data. There remain, however, several fundamental questions which indicate that this model is only an approximation to a better understanding of the Universe. To give a few examples, the Standard Model does not explain the existence of a pattern of quark and lepton masses, does not explain the form of the Cabibbo-Kobayashi-Maskawa mixing matrix, does not give a clue regarding CP violation, and leaves gravity competely beyond its scope.

For an experimentalist, there are two complementary approaches to finding the clues to the unaswered questions in the SM. One strategy is to perform precision tests of SM predictions. Finding deviations would indicate "new physics". Many non-accelerator and low-energy physics experiments belong to this category. Another approach is to look directly for the phenomena beyond the Standard Model by studying the interactions of elementary particles in the highest possible energy range. Topics in this category include Standard Model problems (top quark search, number of generations, $\mathrm{W}$ and $\mathrm{Z}$ mass measurements, Higgs production), and problems which explore physics beyond the Standard Model (higher mass $\mathrm{W}^{\prime}$ o: $\boldsymbol{Z}^{\prime}$, compositeness, supersymmetry, and other exotic phenomena).

The essential factors determining this reach into the unknown are the energy available in the collision, $\sqrt{s}$, and the integrated luminosity $L$. With the closing of the Spp̄S Collider at CERN, the Fermilab Tevatron will be for several years to come the highest energy hadron collider in the world. The CDF detector is a powerful instrument designed to study a wide range of physics in proton-antiproton collisions.

The 1992/1993 Tevatron Collider run has provided experimenters with peak luminosities exceeding $10^{31} \mathrm{~cm}^{-2} \mathrm{~s}^{-1}$ that have resulted in a more than five-fold increase over the integrated luminosity collected in the 1988-1989 run. During the just finished long running period (Ia) of the Fermilab collider (from the summer of 1992 until May 31, 1993), 
CDF accumulated a sample of data corresponding to an integrated luminosity of $L=22$ $\mathrm{pb}^{-1}$. Together with about $L=4.1 \mathrm{pb}^{-1}$ collected in the previous run, in 1988-1989, CDF has now more than $25 \mathrm{pb}^{-1}$. Analysis of this data has given, and will continue to give, physicists a rare opportunity to explore the edge of knowledge.

The list of publications based on the data from the 1988-1989 run which appeared within the last year is quite long (see Section 12), despite the fact that the Collaboration was busy with preparations for the new run and then with the actual data taking.

A series of major upgrades has been proposed for the CDF detector in order to take advantage of the high luminosity expected during the next two runs, Ib - scheduled to begin in November 1993, and then Ic - scheduled for late 1994. A new silicon vertex detector vill replace SVX-I used in the 1992/1993 run, and the CPU power of the Level-3 trigger will be increased four-fold. Those two running periods should bring a five-fold increase in the total integrated luminosity to about $100 \mathrm{pb}^{-1}$. With such a sample of events the prospects of performing precision tests of the Standard Model are very good. The Top-quark, W-mass, $\mathrm{W}-\mathrm{Z}$ mass difference, $\sin ^{2} \Theta_{W}$, and limits on $\mathrm{W}^{\prime}$ and $\mathrm{Z}^{\prime}$ are a few examples of topics which will be studied with CDF.

The primary responsibility of the Tufts CDF Group within the CDF Collaboration, together with Rutgers University and Fermilab, is Level-3 - the third level of the CDF trigger system. The group also participated vigorously in test beam studies, in analysis of 1988-1989 data, and is now taking part in the 1992-1993 run.

\subsection{Data analysis}

Professor Sliwa's physics interests lie in the search for the top quark and in the study of pair production of the intermediate bosons. With data from the 1992/1993 and subsequent runs it should be possible to study the gauge structure of the Standard Model. Di-boson production is sensitive to tri-boson couplings. For example, the process $\mathrm{p} \overline{\mathrm{p}} \rightarrow \mathrm{W} \gamma$ probes the magnetic moment of the $\mathrm{W}$, for which the Standard Model makes a definite prediction.

With other members of CDF Diboson group, Mary Roach-Bellino, Douglas Benjamin, Mark Timko and $\mathrm{K}$. Sliwa finished the analysis of $\mathrm{W}_{\gamma}$ and $\mathrm{Z}_{\gamma}$ events based on the 1988/1989 data. The measurement were presented in CDF Note 1041, which will become a basis of a Physical Review D paper. The cross-sections have been measured, and the analysis programs have been prepared for future, higher luminosity, runs. Mr. Benjamin has presented the results at the International XXVII Rencontres de Moriond, held in Spring 1993 in Les Arcs, France.

The comparison of Monte Carlo events with CDF data for several variables is shown in Figs. 5.1-5.3. The measurents of the $\sigma^{*} \mathrm{BR}(\mathrm{W} \gamma)$ and $\sigma^{*} \mathrm{BR}(\mathrm{Z} \gamma)$ are summarized in Table 1. These results can be translated into measurements of anomalous WW $\gamma$ coupling, $\kappa$ and $\lambda$ (for the Standard Model $\kappa=1$ and $\lambda=0$ ). The contour plot summarizing this analysis is shown in Fig. 5.4.

Mary Roach-Bellino and Doug Benjamin are in the process of writing their doctoral dissertations based on $\mathrm{Z}_{\gamma}$ and $\mathrm{W} \gamma$ final states, with $\mathrm{Z}$ and $\mathrm{W}$ decaying into $Z \rightarrow e^{+} e^{-}$ and $\mathrm{W} \rightarrow e \nu$ respectively. 
Mark Timko, relieved from his responsibilities of maintaining the Level-3 algorithms (the run has ended !), is pursuing vigorously the analysis of $\mathrm{W} \gamma$ events in the new data from the 1992/1993 run.

More generally, studying di-boson production could provide essential information about the mechanism of the electroweak symmetry breaking. The minimal Higgs sector of the Standard Model is regarded by many as quite artificial. There are models proposed in which the Higgs scalar does not exist as an elementary particle, and the symmetry breaking mechanism is of dynamical origin. With integrated luminosities of $25 \mathrm{pb}^{-1}$ and $100 \mathrm{pb}^{-1}$ one should be able to observe the di-boson final states predicted in those models. Finding clues to the mechanism of the spontaneous symmetry breaking is the most important experimental problem in forthcoming years.

With $100 \mathrm{pb}^{-1}$, the top quarks should be found in CDF if their mass is less than 170 $\mathrm{GeV}$. The kinematical analysis technique developed by Prof. Sliwa together with Prof. Goldstein of Tufts and R.H. Dalitz of Oxford University could prove very important in discovering the top quark and measuring its mass.

Professor Sliwa has worked on refining the method, improving its efficiency, and on the analysis of all available CDF data. Most importantly, the effects of initial state gluon radiation were taken into account. The very encouraging results were presented to CDF analysis groups, and were summarized in an internal CDF note (CDF/ANA/TOP/CDFR/1993). Analysis of background sources is underway.

\subsection{Tufts responsibilities in the CDF Collaboration.}

The Tufts CDF Group, together with the Rutgers Group, has taken responsibility for designing, building, and maintaining the Level-3 trigger systems during the 1992-1993 and 1994 CDF runs. This is a very important part of the CDF upgrade program. It is one of the crucial elements without which CDF would not be able to take advantage of the higher luminosities to be available in future Tevatron runs. Professor Sliwa has proposed that the Tufts work on the new Level-3 system be the Tufts Group contribution to the CDF Collaboration. Dr. Timko and Mr. Benjamin are in residence at Fermilab. Mr. Benjamin was responsible for development of the new electron and photon identification algorithm, and he was a librarian for the electron code. All members of the Tufts CDF Group have worked on the debugging and verification of the new Level-3 algorithms which were developed for the 1992/1993 run. The critical issue was whether the complete tracking reconstruction could be done on-line in Level-3 Silicon Graphics nodes. This would enable the electron and muon selection algorithms to work much more efficiently and safely reject enough events to match the data logging capability even at the very high luminosities expected in the new run.

Dr. Timko has taken responsibility for constructing, verifying and maintaining the Level-3 executable code. He has designed and built a set of software tools which create and test the code. Improved revisions of a subset of full CDF analysis code were running in the Level-3 nodes while CDF was taking data. His work was crucial to the success of the 1992/1993 run. His work was especially important when the unexpected increase of the per-event execution time became apparent with increasing luminosity - a result of 
recording multiple interactions in a single crossing. As a result, the amount of on-line computer power necessary to make Level-3 trigger decision was increasing steadily with luminosity, to the point when serious work had to be done in order to reduce the algorithm execution time without compromising the performance of the Level-3 trigger.

Timko was also one of the essential workers who found and solved problems related with moving the entire CDF software from VAX environment to the Silicon Graphics UNIX environment. He has authored several internal CDF notes on subjects related to his work with the Level-3 system.

\subsection{Students and their activities}

Mary Roach has been concentrating on $\mathrm{Z} \gamma$ analysis which is her thesis topic, but she also helped with taking data at Fermilab. The results of her analysis, in which she finds several $\mathrm{Z} \gamma$ candidates, are consistent with the Standard Model predictions.

Douglas Benjamin moved from Tufts to Fermilab in January 1991, and has taken part . in preparations, actual data taking, and analysis of a CDF test-beam run. Benjamin has been participating in the test beam analysis related to the question of how to estimate the probability of a jet to fragment into an isolated $\pi^{0}$, which will look like a $\gamma$, and will be a background to $\mathrm{Z} \gamma$ and $\mathrm{W} \gamma$ searches. He was also deeply involved in software development for both Level-3 algorithms and offline electron identification code. In the last six months Benjamin has been concentrating on physics analysis.

Kristo Karr has joined the Tufts CDF Group this summer. Together with Professor Sliwa, Kristo is studying the $\mathrm{W}+3$ jets and $\mathrm{W}+4$ jets Monte Carlo events generated with the VECBOS program, which incorporates detailed QCD calculation by Berends, Kuijf, Tausk and Giele. The goal is to determine which set of Monte Carlo data describes the CDF data best. Because the $\mathrm{W}+$ jets events are expected to be the dominant background to $t \bar{t}$ events decaying into a lepton and four jets, this study is essential to Professor Sliwa's top search in this channel. 


\section{Signal vs Observed Events $W / Z \gamma$ \\ CDF Preliminary 88-89 Data}

$$
\Delta R_{\gamma \text { lepton }}>0.7\left|\eta_{\gamma}\right|<1.1
$$

Shaded Histogram Signal MC*10

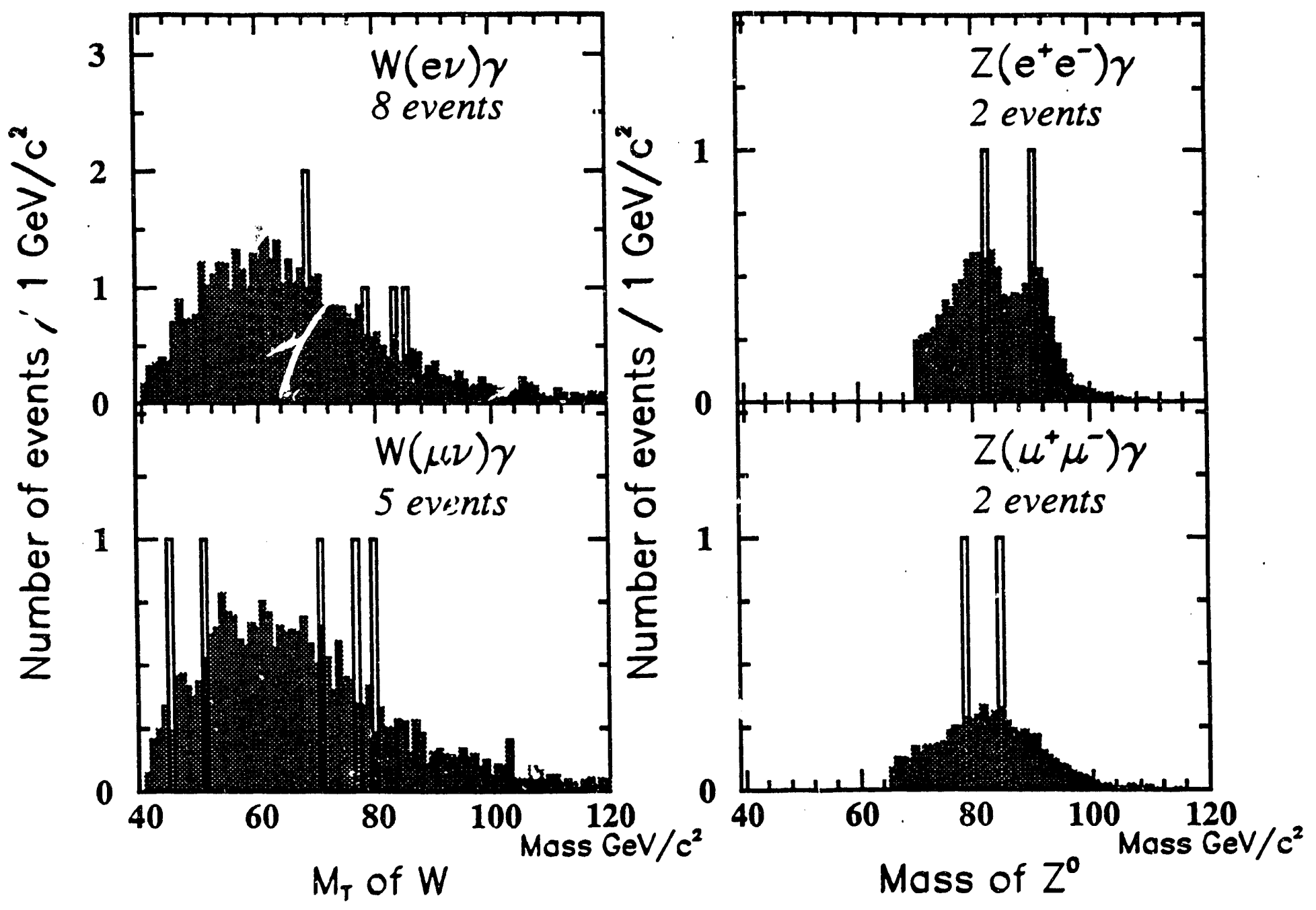

Figure 5.1 


\section{Signal vs Observed Events $W / Z \gamma$ \\ CDF Preliminary 88-89 Data}

$$
\Delta R_{\gamma \text { lepton }}>0.7\left|\eta_{\gamma}\right|<1.1
$$

Shaded Histogram Signal MC

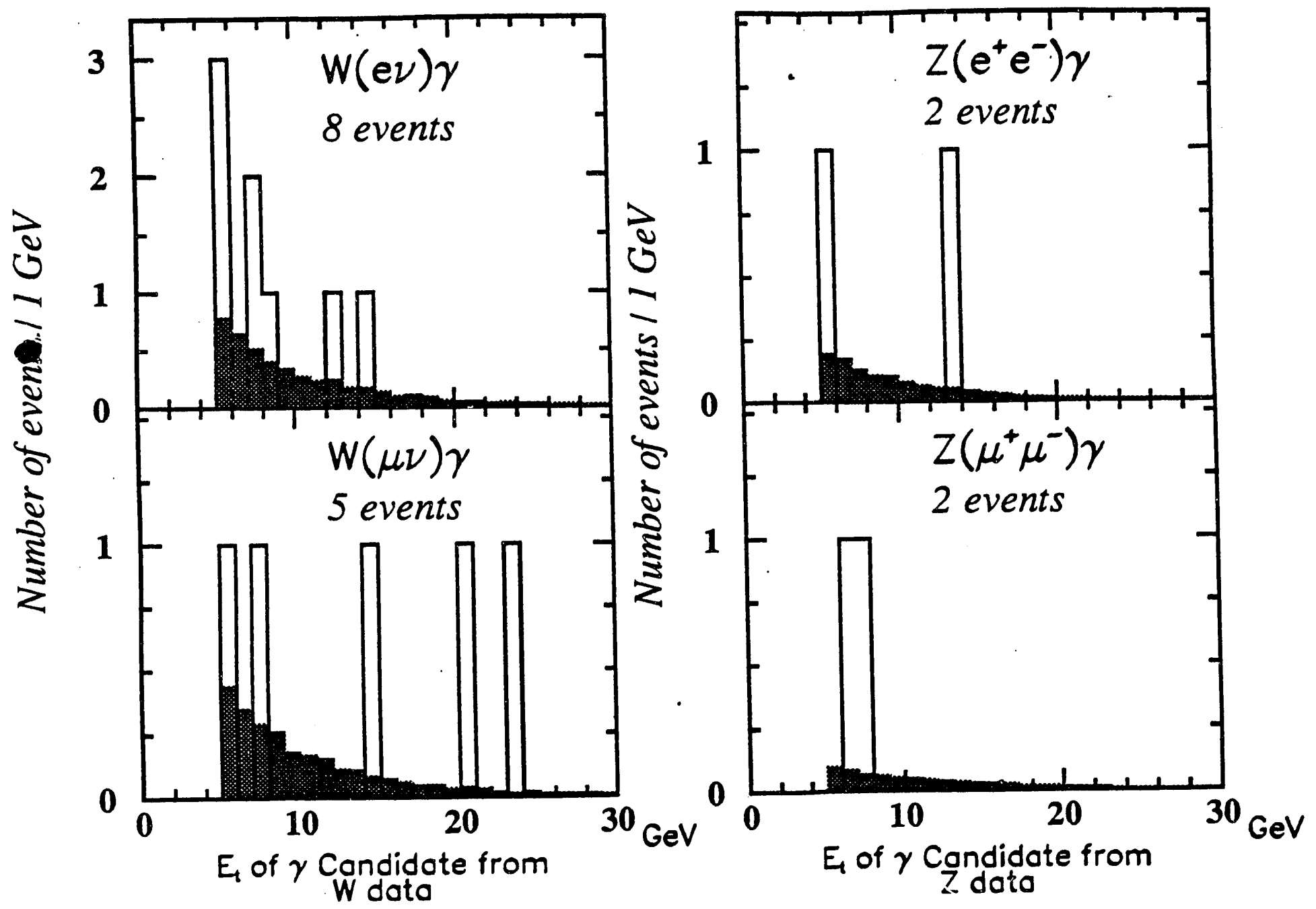

Figure 5.2 


\section{Signal vs Observed Events $W / Z \gamma$ CDF Preliminary 88-89 Data}

$$
\Delta R_{\text {repton }}>0.7\left|\eta_{\gamma}\right|<1.1
$$

Shaded Histogram Signal MC
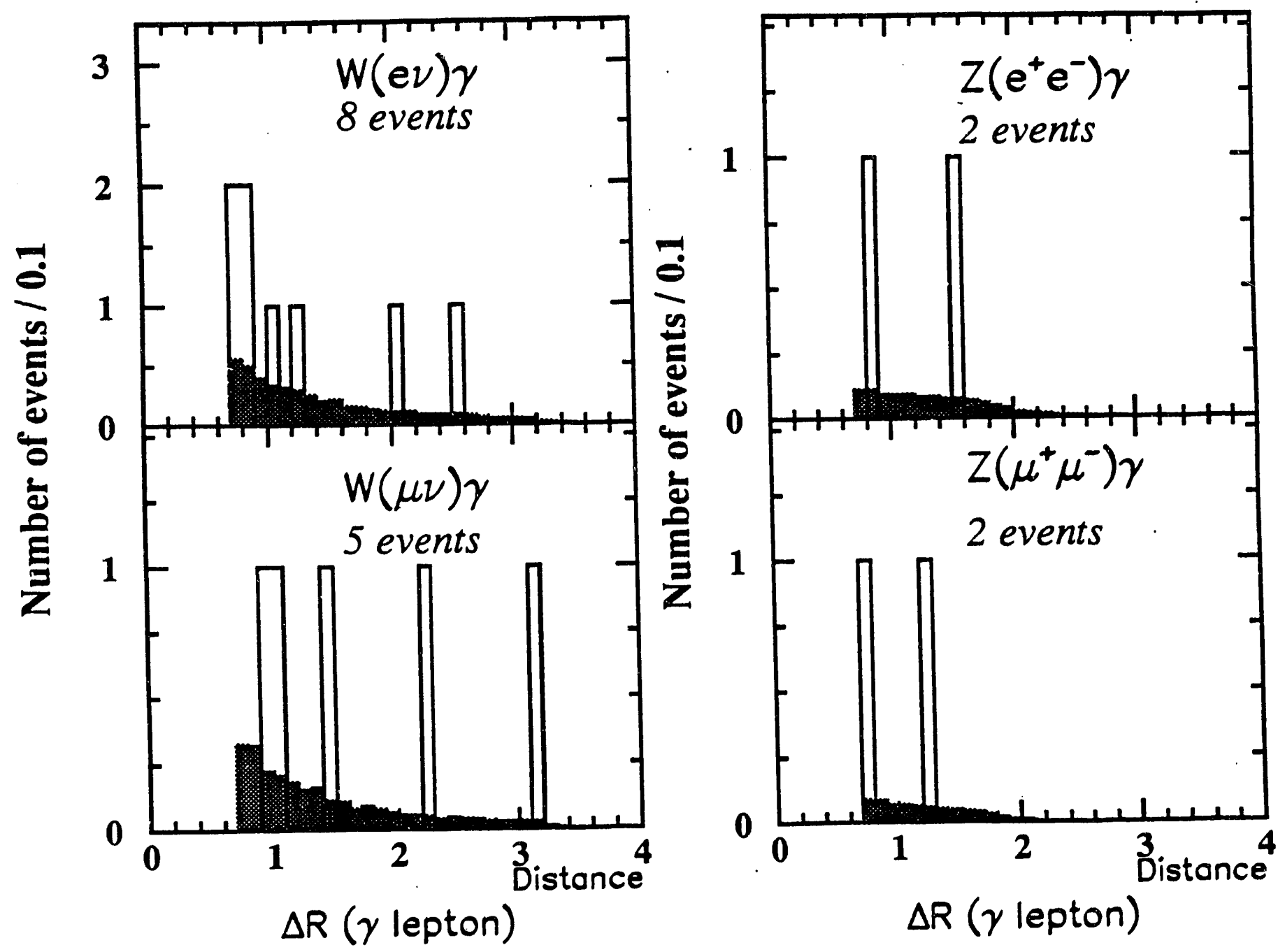

Figure 5.3 




Figure 5.4 
Table 1 . Summary of $W \gamma$ and $Z_{\gamma}$ Results

\begin{tabular}{|c|c|c|c|c|}
\hline Channel & $N_{\text {obs }}$ & $\sum N_{\text {bkgnd }}$ & $N_{\text {signal }}$ & $N_{\text {pred }}^{S M}$ \\
\hline$e W \gamma$ & 8 & $3.8 \pm 0.8 \pm 1.5$ & $4.2 \pm 2.9 \pm 1.5$ & $4.6 \pm 0.4$ \\
$\mu W \gamma$ & 5 & $2.2 \pm 0.4 \pm 0.9$ & $2.6 \pm 2.3 \pm 0.8$ & $2.5 \pm 0.2$ \\
\hline$e Z_{\gamma}$ & 2 & $0.3 \pm 0.1 \pm 0.1$ & $1.7 \pm 1.1 \pm 0.1$ & $1.2 \pm 0.1$ \\
$\mu Z_{\gamma}$ & 2 & $0.1 \pm 0.1 \pm 0.1$ & $1.9 \pm 1.0 \pm 0.1$ & $0.7 \pm 0.1$ \\
\hline
\end{tabular}

Combined $e+\mu \quad \sigma * B R(W+\gamma)$ and $\sigma * B R(Z+\gamma)$ Results

\begin{tabular}{|l|c|c|}
\hline Channel & $\sigma \cdot B_{\text {expt }}(p b)$ & $\sigma \cdot B_{\text {vred }}^{S M}(p b)$ \\
\hline$e+\mu W_{\gamma}(68.3 \% D S C L)$ & $17.9_{-9.8-1.1}^{+9.8}+\frac{1.8}{+4.5}=17.9_{-10.7}^{+11.3}$ & $19.0 \pm 0.3$ \\
$e+\mu W_{\gamma}(68.3 \% S S C L)$ & $<23.5$ & \\
$e+\mu W_{\gamma}(90.0 \% S S C L)$ & $<32.9$ & \\
$e+\mu W_{\gamma}(95.0 \% S S C L)$ & $<37.4$ & \\
\hline & & \\
$e+\mu Z_{\gamma}(68.3 \% D S C L)$ & $9.2_{-5.2}^{+5.2+0.5}+0.4=9.2_{-5.1}^{+5.5}$ & $4.7 \pm 0.1$ \\
$e+\mu Z_{\gamma}(68.3 \% S S C L)$ & $<11.8$ & \\
$e+\mu Z_{\gamma}(90.0 \% S S C L)$ & $<16.6$ & \\
$e+\mu Z_{\gamma}(95.0 \% S S C L)$ & $<19.1$ & \\
\hline
\end{tabular}




\section{The Solenoidal Detector for the Supercollider}

(Profs. Kafka, Mann, Milburn, Napier, Sliwa; Research Technicians McMaster and Dupuis; Graduate Technical Assistants Pfenning and Wall; with the Solenoidal Detector Collaboration.)

The Solenoidal Detector is a large general-purpose detector designed to pursue a broad range of physics goals at the Superconducting Supercollider. The detector's central tracking system, which surrounds the colliding beam interaction region, consists of an inner silicon tracker and an outer, straw-drift-tube barrel tracker together with an array of gas microstrip detectors. The tracking system is contained within a superconducting solenoid that provides a peak field at the interaction point of 2.0 Teslas. The solenoid and tracking system are surrounded by hermetic calorimetry. A large system of magnetized-iron toroids, wire chambers and scintillation counters for muon identification and momentum measurement surrounds the calorimetry. Muon triggering and identification for pseudo-rapidity less than 2.5 are provided by this system; muon momenta are precisely determined by a combination of measurement in the central tracking system and by deflection measurements in the iron toroids. An isometric view of the Solenoidal Detector is shown in Fig. 6.1. ${ }^{1}$

The Tufts group started with the Solenoidal Detector Collaboration (SDC) at its inception in 1989. Our work within the SDC has focussed upon the Muon Subsystem, for which many of the design considerations were familiar to us as a result of our experierce with the Soudan active shield array. During the past three years we have coordinated our activities with the Boston Muon Consortium, which includes researchers from Brandeis, Harvard, Tufts, and C. S. Draper Laboratory. The Boston Consortium has continued to receive funding from the Texas National Research Laboratory Commission (TNRLC) for work leading to development of an SDC precision muon system. Our activities within the Boston Consortium are summarized in the paragraphs below.

\section{A. Spatial precision of SDC muon drift tubes}

The accepted SDC muon drift cell design, as developed at the Univ. of Washington, specifies a circular cross-section with a central sense wire and two planar electrodes to shape the field and provide a relatively linear drifting field in the vicinity of the median plane. These field-shaping electrodes on either side of the sense wire are supported by a dielectric material "Noryl"; the electrodes need not be at the same potential as the wire. Within a drift tube of $4.5 \mathrm{~cm}$ radius, the electrode widths, locations, and voltages are designed to provide quasi-uniform drift regions extending $\pm 4.5 \mathrm{~cm}$ in each direction from the wire. The $4.5 \mathrm{~cm}$ dimension is a limit required to keep the maximum drift time under 1 $\mu s$ (governed by data pipe-line limitations) while using a non-flammable and inexpensive gas such as the Ar- $\mathrm{CO}_{2}$ mixture now under consideration. This choice is itself governed by perceptions of prudence, reliability, and cost in respect to the large scale and potential leakage surface area of the muon assembly.

A cross section view of the SDC drift tube design is shown in Fig. 6.2. Depicted in the Figure are the electric field lines (dotted) and the drift isochrones ( ${ }^{* * *}$ every $50 \mathrm{~ns}$, ooo every $250 \mathrm{~ns}$ ) for the case where both sense wire and field shaping electrodes are operated 
at $6200 \mathrm{~V}$. Since $1 \mathrm{~ns}$ corresponds to about $50 \mu \mathrm{m}$, it can be seen from the curvature of the isochrones that at most only a few millimeters of ionizing track will contribute to the leading edge of a signal providing a $100 \mu \mathrm{m}$ position resolution.

\section{Effects of gravitational and electrostatic forces}

The spatial precision attainable using the SDC drift chambers depends upon three factors: (i) the precision of the location of the two ends of the sense wire where they pass through crimp tubes embedded in endcaps as much as nine meters apart; (ii) knowledge of the wire configuration between the ends as governed by gravitational and electrostatic stresses and by the wire tension; and (iii) the uncertainty in the positions of the field shaping electrodes and of the outer drift tube wall with respect to the central wire, following tube assembly into larger modules. Theoretical investigation of factors (ii) and (iii) was carried out by Tufts researchers during 1992, based upon electrostatic modelling. The anode wire if displaced from the electrically neutral point of the drift cell (center of tube) will experience an electrostatic force which will displace it further. The significant displacement occurs along the direction of the drift field. A description of the electrostatic forces and calculations of the effect in $90 \mathrm{~mm}$ drift tubes was reported by us in Refs 2,3 . We found at the operating voltage of the SDC drift tubes that the electrostatic force increases the wire displacement by approximately $13 \%$. Corrections for the electrostatic displacement can be made in the off-line analysis, provided that the position of the wire relative to the tube in the absence of electric fields is known. For gravitational sag of the wire and systematic deformations of the module, the correction is straightforward. Random displacements of the electrnstatic neutral point will result in electrostatic displacements of the wire which cannot be corrected in the off-line analysis and thus contribute directly to measurement error. Such random displacements can result from either imperfections in the tube shape as manufactured, or from not controlling the straightness of the tube during installation (epoxying) in the module. ${ }^{4}$

\section{Effect of tube cross section variations}

The extrusion specifications for the barrel SDC muon tubes include a limiting tolerance for "ovality". This tolerance presently permits a maximum fluctuation \pm 0.015 in. from the nominal outside diameter - $3.685 \mathrm{in} .(93.60 \mathrm{~mm})$ - of the tube. This requirement may exceed the state of the art economically achievable by manufacturers; a relaxation of the ovality tolerance might therefore save considerable money as well as widen the field of potential vendors. While the ovality tolerance is driven by a variety of factors, the most important is the need to maintain measurement precision. A simulation study of the effects of ovality fluctuations upon a tube's ability to measure track locations precisely was carried out by Prof. Milburn and has been submitted as an SDC Note (Ref. 5). He examined the effects of small perturbations in the cross section of the barrel muon tube extrusions from perfect circularity of inner diameter $9.0 \mathrm{~cm}$ centered on the anode wire. The distortions examined include: elliptical extensions and compressions along and perpendicular to the drift axis, with and without conservation of the circumference, radial variations, and "sinuosity" in which the tube deviates from straightness while remaining circular in cross section. The specific case examined was for $\mathrm{ArCO}_{2}$ (88-12) gas and 6200 
volts on the anode wire and electrodes. With linear distortions $\pm 0.030 \mathrm{in}$. $(0.76 \mathrm{~mm})$, twice the current tolerance, the induced measuremen: imprecision in track location varied from the worst case \pm 50 microns for radial expansion and contraction, to less than 6 microns for variations leaving the circumference constant. Thus, comparing to the nominal \pm 0.250 $\mathrm{mm}$ tolerance, the resulting imprecision effects appear to be negligible. It must be noted however, that this favorable conclusion depends strongly upon the assumed operation of the tube on the horizontal plateau of the drift . xity versus field curve. The tube cross sectional distortions examined have the primary effect of altering the mean electric field along the drift path, and their effect will increase with the slope of that portion of the velocity curve in use. ${ }^{5}$

\section{B. Instrumentation for manufacturing}

The Boston Consortium is preparing two factory assembly lines, one at Tufts and another at Harvard, which will manufacture and test drift tubes of the SDC muon system design. Significant progress was made during the past year in developing instrumentation and in proving out procedures for the numerous fabrication tasks required. Our contributions are outlined below.

\section{Heat-bonding of endcaps onto tubes}

Prototyping to enable the heat-fusion of extruded aluminum drift tubes to their endcaps was carried out at Tufts. An initial Brandeis heat clamp design was revised; the modified assembly consists of eight, separately floating heat pads housed in two semicircular elements. The elements slide together to surround the circumference of a drift tube to be heated. Extensive trials, in which drift tubes were heated and allowed to shrink (via cooling) onto inserted endcaps, were carried out using the setup shown in Figs. 6.3a and $6.3 \mathrm{~b}$. Initial tests established the range of mechanical interference between tube and endcap diameters for which reliable bonding can occur. Follow-up testing showed that the endcap-tube bond was not gas-tight to a desirable level. The situation was remedied by adding grooves with 0 -rings into the endcaps, yielding bonding which is very gas-tight while remaining mechanically strong.

\section{Tube boring and machining}

A drift tube boring and machining area has been set up at Tufts and outfitted with two massive horizontal boring machines. It is envisaged that our shop will carry out all of the boring and machining required for the 9-meter long drift tubes to be assembled by the Boston Consortium for the prototype muon supertower. A tube boring tool was developed; it is outfitted with precisely mounted cartridges which hold replacable blade inserts. The blades, which can be contoured, are rated to be optimal for cutting aluminum. Each insert blade is a triangular piece. To replace a cutting surface, one just rotates the blade - the mounting cartridge ensures that the location of the new blade surface is properly positioned. A central stem which holds the cartridges was made using aluminum, and trial borings were carried out with excellent results. The fabrication of production boring cutters having cylindrical stems of machined steel was coordinated between the Tufts and Harvard shops. A general problem to be addressed is the finish-cutting of drift tubes to precise lengths. To provide this capability, we outfitted our horizontal boring 
station with a digital encoder to provide the operator with an overall (relative) length measurement. Our plan is to have the operator machine each tube down to a preset halt point. The halt point for a particular batch of tubes to be bored and machined-to-finish is established on the station using reference tubes or using a reference bar containing precisely enscribed intervals. A set of five 28-ft long drift tubes was bored and machined at Tufts and then shipped to a commercial vendor for plating. Three of the tubes were cleaned before shipment; two were shipped with the aluminum chips and shavings from the boring operation left inside the tube interiors. When the tubes are returned - hopefully by mid-June - the cleanliness of the plated tubes will be evaluated. In this way we will determine to what extent the drift tube extrusions need to be cleaned prior to plating and prior to final assembly.

\section{Drift tube assembly benches}

Essential to the drift tube assembly line are the assembly benches which hold most of the fabrication instruments, e.g. the endcap heatirg and insertion units, the motor-driven crimping tool, the wire dispenser, and the wire tensioner. Layout schemes proposed by Prof. H. Wellenstein of Brandeis were reviewed by consortium physicists using a wooden mock-up made at Tufts. Assembly fixtures are arranged to be in precisely defined locations when in use, but can be translated away from the tube opening as needed to facilitate other tasks. It is essential to avoid building in a preferred "handedness" to the assembly benches (e.g. a setup convenient for right-handers but awkward for lefties). The benches are massive, but are mounted on ball-bearing rollers which move on two rails. Consequently the distance between benches which service the ends of the tubes can be varied so as to accommodate all tube lengths of relevance to the SDC barrel region (6 to 9 meters). Mounted onto the tube assembly benches are massive six-jaw chucks - one to each bench. The chucks provide a precise, firm hold at the drift tube ends and force each end to be circular, as required for reliable insertion of endcaps. Planning is underway concerning layout and fixturing required for quality assurance measurements to be obtained for each drift tube before it leaves the assembly bench. It is envisaged that power supplies for quality assurance instrumentation can be mounted underneath the benches. However, devices which need to be read or keyed could be mounted on a movable frame which is suspended from a track from the clean room ceilng.

\section{C. Organization}

Researchers from the Boston Consortium institutions have met weekly - Monday mornings - for the past two and one-half years, and a harmonious coordination of effort has been achieved. Since November 1992, the Consortium held two three-day meetings with the Univ. of Washington group for the purpose of reviewing work leading to the construction at the SSC laboratory of the first supertower for the muon barrel system. These meetings, the first in Seattle and the second in Boston during March, were very productive and are likely to be continued in the near future.

\section{Section 6 References}

1. "SDC Technical Design Report", Tufts authors, with the Solenoidal Detector Collaboration, SDC-92-201, April 1992. 
2. R. Milburn, "Electromechanics of Drift Tube Wires", SDC-92- 320, Sept. 1, 1992.

3. G. Yasuda and R. Milburn, "SDC-9cm Muon Drift Tube: Electromechanical StudiesI", SDC-92-321, Sept. 3, 1992.

4. Excerpted from draft of "Tube Design Report", H. Lubatti et al., June 1993; p. 8.

5. R.H. Milburn, "Ovality Tolerances and Muon Tube Precision", SDC-93-519, June 1, 1993. 


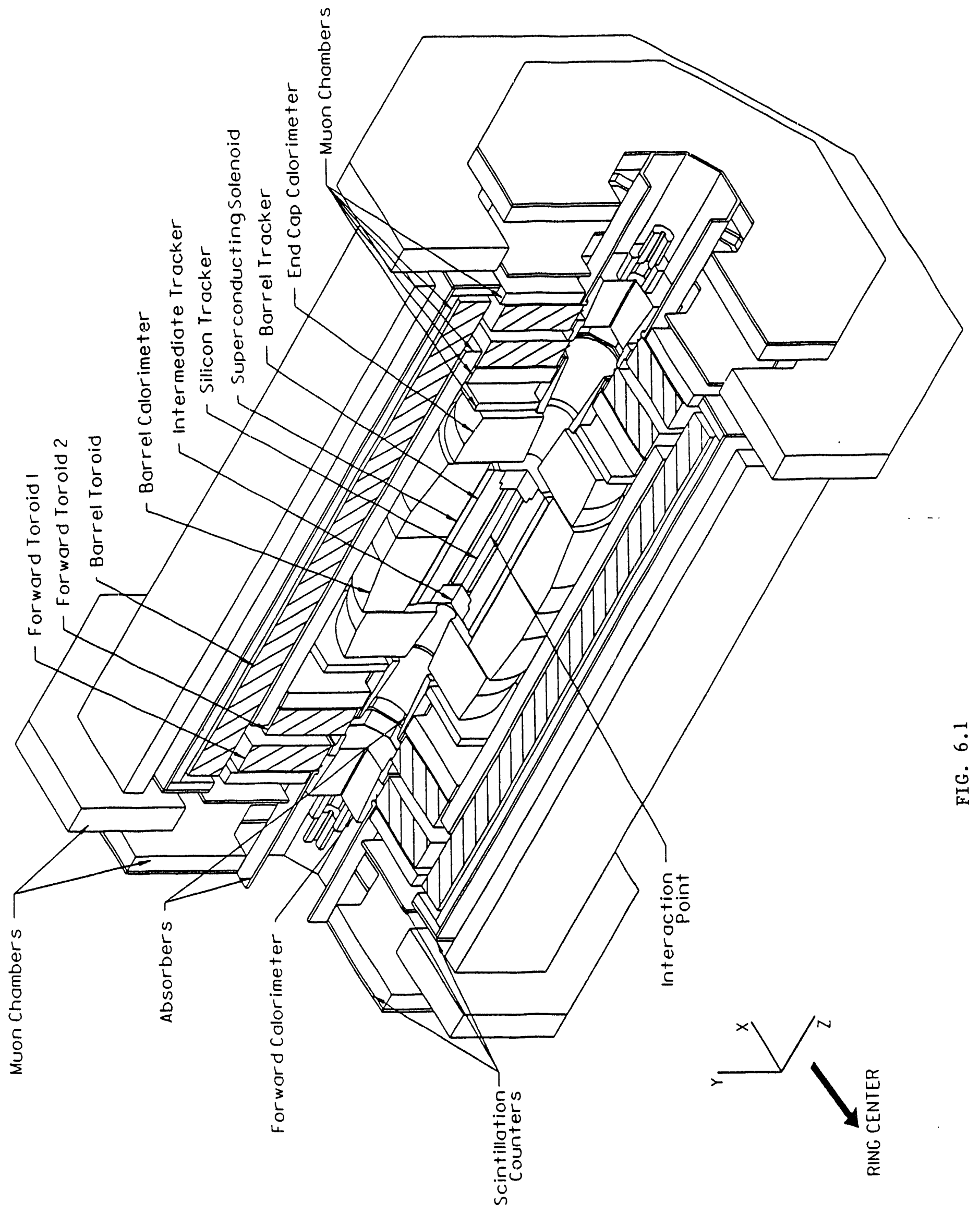




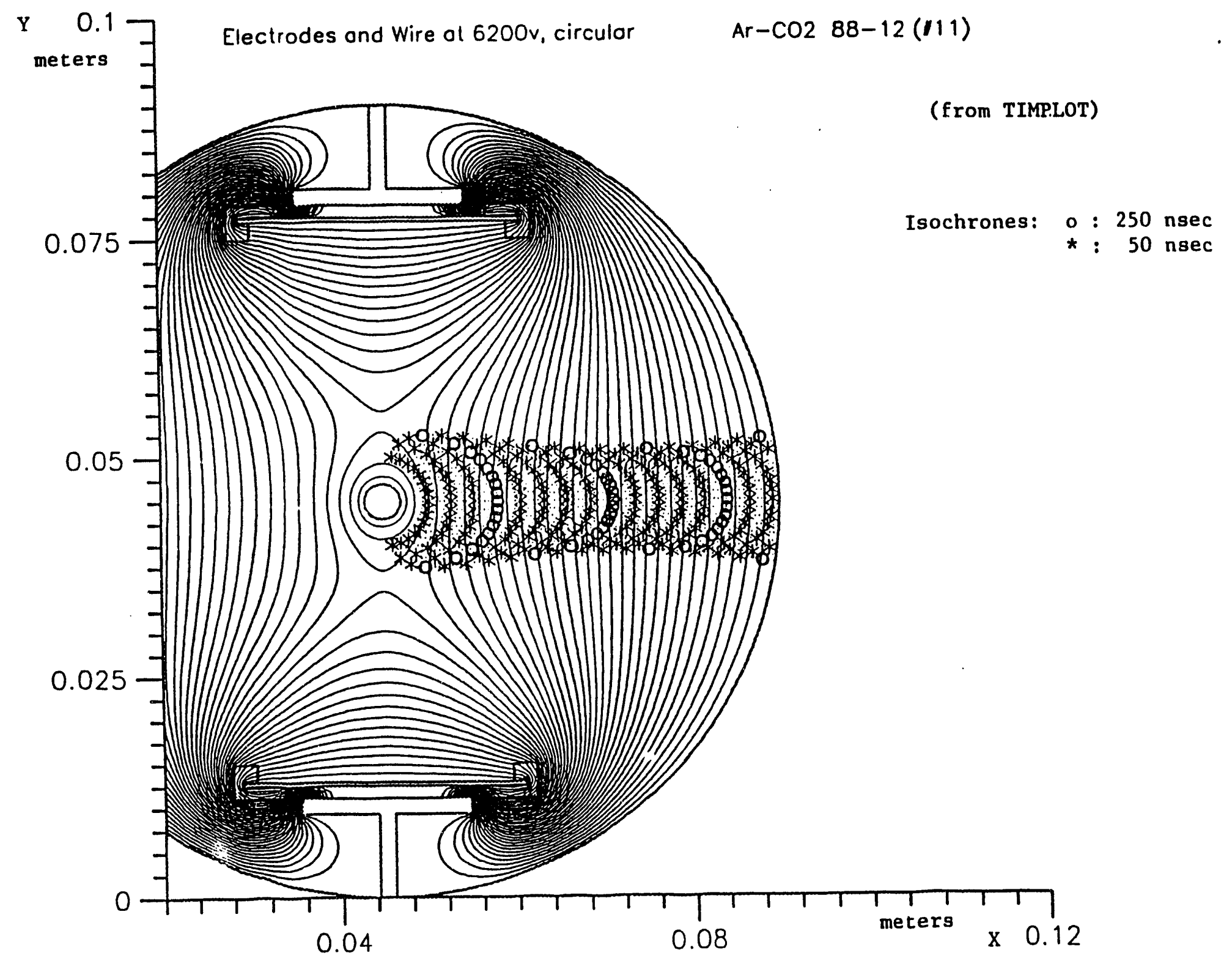

FIG. 6.2 




FIG. $6.3 a$

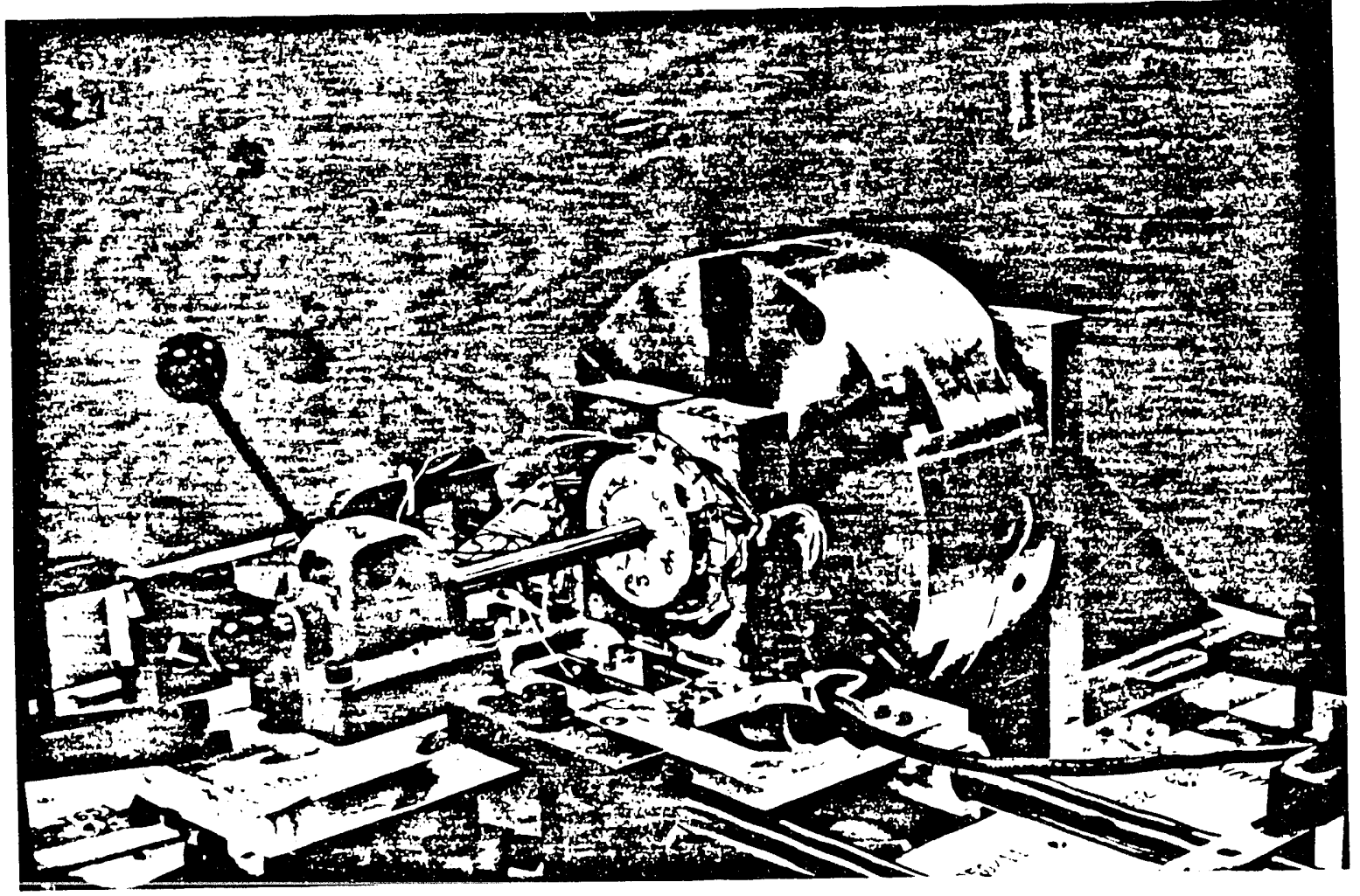

FIG. $6.3 b$ 


\section{Neutrino telescope proposal}

Profs. Kafka, Mann, Milburn, Napier, Oliver, Schneps and Sliwa in collaboration with groups from Saclay, CESNEF-Milano, Arkansas at Fayetteville, Laboratorio Nazionale del Gran Sasso, UC at Irvine, KEK, Arkansas at Little Rock, LSU, Maryland, Neuchatel, Padova, Pavia, Tokyo, Torino, Warsaw, Wisconsin and Zaragoza.)

Tufts is a member of an international collaboration working toward obtaining funding for the construction in Italy of a very large $\left(10^{5} \mathrm{~m}^{2}\right.$ sensitive area) water Cherenkov detector of high energy neutrinos. The physics and astrophysics goals and the proposed design of this project were reviewed extensively in our Progress Report of July 1992.

This neutrino telescope proposal, known as NET, was submitted to the agency INFN of the Italian government in March 1992. Two possible sites, both former quarries, near the Gran Sasso Laboratory were recommended. The overall budget is about $\$ 100 \mathrm{M}$, of which $70 \%$ is for civil construction. A decision by INFN on whether to fund this project has been deferred, and we have no information on when a decision will be forthcoming. 


\section{THEORETICAL PROJECTS}

\section{Polarization in massive quark and hadron production}

(Prof. Goldstein, with W.G.D. Dharmaratna, Florida State University)

In QCD with massless quarks, the quark helicity is conserved; there can be no single quark polarization in any perturbatively calculated scattering process. It is known experimentally that inclusively produced hyperons carry significant polarization. Are these seemingly contradictory conclusions reconcilable? Several years ago we developed a QCD based theoretical model for the production of polarized massive quarks and hadrons. ${ }^{1}$ It was reasoned that because the hyperons carried strange quarks, the massless condition was broken, and the null result for polarization would be changed at the quark level. If the effective mass is large compared to the scale of momentum transfers involved in the actual data, then QCD beyond tree level predicts single quark polarization effects. We found that gluon fusion was the principle source of such effects, by explicitly calculating the one-loop corrections to t'ie QCD sub-processes. Over the last two years we compiled all other relevent sub-processes, still finding the gluon fusion dominant. ${ }^{2}$

The original purpose of the calculations was to explain the large hyperon polarization. To go from s-quark polarization in hard QCD at the parton level to hadron polarization required some scheme for recombination or fragmentation of the s-quark. We used a simple model for the recombination of the s-quark with the isoscalar u-d "diquark" from the proton beam that assured that the Feynman $\mathrm{x}$ of the hyperon not exceed its kinematic bounds, while not diluting the quark's polarization. There resulted a kinematic dependence that mimicked the data on $\Lambda$ production (the best studied of the hyperons). However, the predicted magnitude remained a factor of about $2 \pi$ too small. This shortfall was not overcome by including quark annihilation processes.

One difficulty underlying our application of QCD perturbation theory to the production of s-quarks is that the quark mass, either current or consticuent (or even hadronic), is not large compared to the QCD scale parameter $\Lambda_{Q C D}$. That suggests that nonperturbative effects are important even at the subprocess parton level. Non-perturbative contributions have been modelled in many ways by other groups, although with considerable arbitrariness and mixed success. It may be that "ioft" physics dominates the hyperon production after all, ${ }^{3}$ but the regime in which there is a transitjon to "hard" physics and decreasing polarization has not been reached. ${ }^{4}$

It is anticipated that the results of our calculation at the parton level will be applicable for heavier mass quarks and hadrons. For top quark production, with top mass near 140 $\mathrm{GeV} / c^{2}$, the formula we obtained has been used to predict the polarization at the Tevatron and the SSC. ${ }^{5,6}$ The largest polarization occurs ai $x_{F} \simeq 0.5$ and for massless quarks in the initial state. Note that the top quark polarization from gluon fusion depends on all the other quark masses due to the contribution from the vacuum polarization diagram. With the running coupling constant of $\alpha_{s}=0.15$, the magnitude is small, $\sim 2 \%$ from gluon fusion and $\sim 4 \%$ from annihilation at the peak. Nevertheless, it is not zero, even in the kinematic region where perturbative QCD is expect ad to be valid with no doubt. This is an interesting result to verify experimentally, since the predicted pclarization arises 
from the one loop level but not as a correction to the leading order predictions. Such a measurement will be a direct test of the fourth order prediction from perturbative QCD.

The other obvious caveat in our model for hyperons involves our ad hoc treatment of the recombination of the massive quark with the beam diquark. While such a model has some phenomenological appeal, it cannot be implemented within the rules of QCD perturbation theory. It has been shown recently ${ }^{7}$ that single hadron polarization can be defined as a factorizable process, partially calculable, in principle, in QCD, providing the kinematic constraints for fragmentation of the relevant quark are satisfied. In the processes in question here, polarized quark fragmentation factors out of the hard sub-process production cross section. We have been studying the implications of this simplification for polarized b- and t-quarks and their fragmentation. For top quark masses above 120 $\mathrm{GeV}^{6,8}$ which was suggested in our own work, the top quark decays before it hadronizes, so that its weak decay is a straightforward polarization analyzer. 


\section{Production characterisitics of top quarks}

(Prof. Goldstein, with R.H. Dalitz, University of Oxford)

In several papers over the last two years we developed a kinematic method for identifying top quark production from $\bar{p} p$ collisions and for determining the mass of the top..$^{6,8} \mathrm{We}$ continued to refine that method this year with particular emphasis on making the mass determination very precise. To summarize the method first, we used a geometric construction for determining the momentum of a top quark, given the observation of its decay via its semi-leptonic channel into a high energy lepton and a b-jet, with an unobserved neutrino. The two-step decay $\mathrm{t} \rightarrow \mathrm{b}+\mathrm{W}^{+}, \mathrm{W}^{+} \rightarrow l+\nu$, leads to the construction of a paraboloid surface of possible top three-momenta, based on the momenta of the b-jet and the lepton. Each possible top mass corresponds to an elliptical cross-section of the paraboloid. The position on the ellipse parameterizes the unmeasured neutrino momentum. For the production of a pair $t \bar{t}$, there are two such ellipses for each value of $m_{t}$.

A t $t \bar{t}$ pair is produced by the interaction of a parton of the proton with an antiparton of the antiproton, each parton having small transverse momentum. The simplest parton model was assumed ${ }^{8}$, requiring that the net transverse momentum of the produced pair be zero, to analyze the one published candidate event from the CDF data of 1988-89 ${ }^{9}$. For that situation, the projections of the two ellipses (at a given $m_{t}$ ) onto the transverse momentum plane, with the anti-top ellipse inverted, must lead to intersections. The analysis showed that the event was consistent with the production and decay of a top quark of mass value of 120 to $153 \mathrm{GeV}$, with a central value of $131 \mathrm{GeV}$. An earlier analysis, that allowed a range of total transverse momenta, $p_{T}$, of $0.1 m_{t}$, had yielded 114 to $144 \mathrm{GeV}$. $^{6}$

We have since allowed for all $p_{T}$ values by weighting the probability for each $p_{T}$ with a Gaussian distribution. ${ }^{10}$ We also include the smearing of the measured jet momenta over experimental uncertainties, and integrate over the weighted range of those uncertainties. That integration shifts the peak probability for that caudidate event to a somewhat lower value of $m_{t}$. To study how robust the method is for other configurations, we used an unofficial preliminary measurement of a dilepton event found in the 1992-93 run at the Tevatron (shown at the DPF Meeting of Nov. 1992). That event yielded a higher value of mass, $m_{t} \simeq 150 \mathrm{GeV}$. Combining the two events, as if they were real top events, we found them only barely compatible, overlapping in the region around $135 \mathrm{GeV}$. If this second event were a real, correctly measured event, we would have to conclude that either one of the events was not a top production, or that there was a large fluctuation in one of the kinematic variables (e.g. the $p_{T}$ was unusually large).

We also began the work of including the $t \bar{t}$ spin correlations in the evaluation of the production and decay probabilities. As outlined in last year's report, the $q \bar{q}$ and gluon fusion subprocesses favor opposite correlations for the produced $t$ and $\bar{t}$ spins. Our previous work on top quark properties showed that for the anticipated masses, the parity violating weak decays will dominate, and thereby provide good polarization analyzing power in the decay angular distribution. ${ }^{6}$ With Prof. Sliwa, simulations of the various kinematical enhancements that can result from the spin correlations were studied, although with ambiguous results. This year Goldstein and Dalitz included the relevant density matrices in their geometrical method and started to compare the results on different simple 
simulations that incorporate nin effects. This work is in progress.

When a $t \bar{t}$ pair is produ the decay channel with a single high $p_{T}$ lepton accompanied by high $p_{T}$ jets has a $!=$ er probability than the dilepton and jets channel. One of the $\mathrm{W}$ bosons that is formed in the pair of decays will itself decay into quark jets. So the single lepton with 4 or more jets is a propitious channel to study. For that reason, along with Prof. Sliwa, we extended our geometric method of analysis to these configurations, as reported last year. ${ }^{11,12}$ Goldstein and Dalitz continued to refine and extend this work to make the method a good and reliable discriminator for mass determination. The following will summarize the work done in collaboration with Prof. Sliwa. The subsequent work done by Profs. Goldstein and Dalitz will be discussed thereafter.

The extended method proceeds as follows: Three high $p_{T}$ jets are chosen to be associated with the hadronic decay of one tentative top quark. Their 4-momenta determine a total 4-momentum for the top. The momentum $\vec{p}_{t}$ and the mass $m_{t}^{*}$ are determined. The lepton and remaining candidate b-jet 4-momenta, along with the mass $m_{t}^{*}$ just determined, define an ellipse of possible 3 -momenta $\vec{p}_{t^{\prime}}$ for the other top quark.

Having a tentative assignment of an event to the $t \bar{t}$ hypothesis, the a priori top production probability is then calculated. Associated with each $\vec{p}_{t^{\prime}}$ is a decay probability. Associated with the transverse part of $\left(\vec{p}_{t^{\prime}}+\vec{p}_{t}\right)$ is the total transverse momentum, which was limited by a fraction of $m_{t}^{*}$, as in the original dilepton calculation. ${ }^{6}$ To complete the probabilistic weighting for any particular "matched" kinematic configuration, the production mechanism probability favored for that particular kinematic match is folded in. That assignment depends on the Bjorken $x$ values for the initiating partons, the structure functions $F_{1}\left(x_{1}\right)$ and $F_{2}\left(x_{2}\right)$, and the parton subprocess center-of-mass energy and momentum transfer through the cross section.

A further complication in evaluating real data is due to the sizable uncertainty in jet momenta measurements. For a perfectly measured event, the task of checking whether or not the top production hypothesis is likely to be correct is straightforward. The likelihood for that event, with a specific identification of jet flavors, would be achieved by simply evaluating the a priori probability for the given kinematic configuration and applying Bayesian statistics. In this fitting scheme, uncertainty in the jet momenta is incorporated by allowing the jet energy to vary over a range of values determined by the empirical probability distribution for jet energy uncertainties. The different values of jet momenta are weighted by that distribution - an integral is approximated. The lack of flavor identification for the jets is another complication. This makes it necessary to consider all possible jet combinations in a given event and to sum their probabilities. The kinematic restrictions favoring small net transverse momentum for decay products and requiring one jet pair to have invariant mass near the $\mathrm{W}$ mass, enhance the probability for correct jet combinations.

Last year the fitting procedure was tested on several kinds of simulated events: simulations of top pair production events (a "toy model" and an ISAJET Model Monte Carlo simulation) and a simulation of the expected Standard Model background contribution to the lepton and jets final states. The tests showed that the method was a good discriminator, effectively distinguishing $t \bar{t}$ production from Standard Model background. The probability distribution as a function of top mass showed peaks for top events where they should be 
- masses were well determined. The Standard Model background peaked at low mass and gave virtually no substantial contribution in the region above $120 \mathrm{GeV}$. If real events contained a top contribution in the mass range above $120 \mathrm{GeV}$ (as suggested in the dilepton event), we concluded that this procedure would provide a significant demonstration of the existence of such a top quark.

The technique was applied to the data sample recorded during the CDF 1988-1989 run, as noted in the 1992 Progress Report. Preliminary results were encouraging and were presented at internal CDF Heavy Flavour Group Meetings (internal CDF report CDF1751). The results were scrutinized by members of the CDF Collaboration, who decided that the selected events lacked sufficient significance in view of the lack of CDF background studies.

Since that time, Goldstein and Dalitz continue to refine this work. We have scrutinized some of the simulated and candidate events with particular attention to the stability of peaks in the probability distributions ${ }^{10}$ under various assumptions. The total transverse momentum is allowed to vary far from the zero point, while the probability for each $p_{T}$ is weighted by a Gaussian distribution of width proportional to $m_{t}^{*}$. As with the dilepton events that were studied, the peaks can shift and be narrowed or broadened with changes in this distribution. This effect was explored with new simulated "toy model" events. ${ }^{10}$ Of particular concern is the effect of additional hard gluon jets on the transverse momentum distributions. This complication is important and its simulation is being studied also. Different jet energy correction schemes are being used to allow for different assumptions about detection abilities. The original work used now outdated structure functions. We have used updated functions and have noted the shifts that result in various probability distributions. These investigations continue. 


\section{Scattering, spin dependence and mass corrections in Skyrmion models}

(Prof. Goldstein, with graduate student L. Gamberg)

Work continued on the determination of an appropriate Hamiltonian for the extraction of partial wave amplitudes in the Skyrmion model of the nucleon, as well as on quantum mass corrections in topological soliton models. Further, progress was made on questions concerning the gauge invariance of collective coordinate quantization in soliton models.

In the investigation of soliton quantization ${ }^{13}$, modification of the quantization algorithm is necessary due to constraints that arise as a result of introducing collective soliton coordinates (whether employing a canonical quantization or Feynman path integral approach). In the case of canonical quantization, the fundamental commutation brackets must be replaced with Dirac brackets and in the Feynman path integral approach the unrestricted generating functional of Green functions is replaced with a variant of the Fadeev-Popov ${ }^{14}$ functional integral. Both approaches lend themselves to the interpretation that quantization of constrained systems is actually the same problem as quantizing systems with internal gauge symmetry. Hence, one must isolate the physical degrees of freedom via gauge fixing conditions. As with all gauge symmetric systems, there exists freedom in the choice of gauge with the requirement that all physical calculations should be independent of this choice.

In the process of calculating the quantum corrections ${ }^{13,15}$ to the classical soliton mass it has been noted that different gauge choices result in a variation of both the form of the leading order meson soliton interaction vertex and its coupling strength (in terms of $\left.\left[1 / n_{\text {color }}\right]\right)$. If the collective coordinate quantization method is to be considered a reliable method of quantization, it is necessary that calculations of physical observables yield results independent of choice of gauge. As a result, comparison of the calculation of the quantum mass corrections in the different gauges (to a given order in $\left[1 / n_{\text {color }}\right]$ ) becomes a test of the validity of gauge invariance of collective coordinate constrained quantization. Assuming this to be the case, it must be that the different gauges dictate a reorganization of the perturibation series in calculating physical observables.

This test is underway and is expected to shed light on both the ambiguity mentioned above concerning the variation of the leading order interaction with gauge condition, ${ }^{13,15}$ and the concern that the results of calculating physical observables varies with gauge choice.

The infinite color limit of QCD is equivalent, at low energies, to an effective nonlinear chiral scalar field theory, admitting the possibility that the nucleon and other excited fermions can be identified with soliton-like solutions to that field theory. ${ }^{16}$ The spin dependences of observable quantities calculated from this Skyrmion model are of particular interest. This was addressed initially by a determination of the pion-nucleon phase sinfts, ${ }^{17}$ although not completely successfully. The disagreement was attributed to the presence of zero modes in the amplitudes. Several questions were raised by that work. How are the zero-modes removed? How are next order terms generated via non-static corrections and the $1 / n_{\text {color }}$ expansion? What is the proper quantization scheme for an extended object in field theory? 
To address these issues we have pursued a formulation in terms of a symmetric nonrigid gauge quantization scheme. ${ }^{18}$ This method clearly addresses the subtle connection between constraints due to collective coordinate quantization and zero modes. It is found that this formulation significantly alters the $1 / n_{\text {color }}$ properties of the theory. In particular, the effects of this quantization scheme arise at order zero in the $1 / n_{\text {color }}$ expansion resulting in modification of the meson distorted waves from the Skyrmion backround equations. This refinement further translates into a modification of the background phase shift calculations of $\pi N$ scattering.

Motivation for this particular scheme was provided by first considering a simpler system: explicitly removing the translation mode in the $1+1$ dimensional non-linear $\phi^{4}$ field theory in the soliton sector using various quantization schemes. ${ }^{19}$ That procedure was checked with the transition matrix for the excited soliton transition amplitude.

Given a procedure for choosing a gauge, forming a sensible interaction vertex and generating a Born approximation, the problem of how the ever present background soliton effects the scattering states remains. We have used the Distorted Wave Born Approximation to set up the appropriate vertex for the scheme of Holzwarth ${ }^{20}$. There are other credible schemes for choosing the gauge and removing zero modes that we have compared. It is expected that the resulting $s$ - and p-waves will differ significantly from the plane wave approximations, although it can not be stated that the modifications will be favorable until the numerical calculations are completed.

It has also been noted ${ }^{21}$ that in calculating the quantum mass corrections of the quantized Skyrmion (which is determined by an energy integral over the backround channel phase shifts) that the low energy behavior of the background phase shifts is significantly influenced by the zero modes in the theory (their existence is a result of the symmetry operations which transform the family of classical soliton solutions into one another). This calculation vas first performed ${ }^{21}$ subject to the naive quantization scheme that did not take into accuunt the modification of the background phase shifts due to constrained quanization. Further it is noted ${ }^{13,15}$ that the symmetric non-rigid gauge pushes the order 0 (in $1 / n_{\text {color }}$ ) zero mode interaction out of the background equations enabling one to isolate and identify it as an interaction lagrangian term. Being order zero requires nonperturbative methods to account for its effects. (For instance in calculating the quantum corrections to the soliton mass).

The loop approximation techniques ${ }^{22}$ (first utilized in approximating the effective potential in QFT) lends itself to this task by "summing up" the order zero effects of the zero mode interaction lagrangian. This calculation is underway ${ }^{13,15}$ and is expected to complement and possibly significantly modify the more naive quantum mass correction results obtained using the perturbative methods of Holzwarth ${ }^{23}$ and Moussallam. ${ }^{24}$

\section{References for Sections 8, 9 and 10}

1. W. G. D. Dharmaratna and G. R. Goldstein, Phys. Rev. D41, 1731 (1990); G. R. Goldstein, W. G. D. Dharmaratna, and G. A. Ringland, Z. Phys. C 41, 673 (1989); "Gluon fusion as a source for massive quark polarization", W. G. D. Dharmaratna, and Gary R. Goldstein, A.I.P. Conference Proceedings 187, 744 (1989); Phys. Rev. 
D41 Rapid Comm., 731 (1990); W. G. D. Dharmaratna, "Massive Quark Polarization in QCD Subprocesses", Ph.D. Dissertation, Tufts University, Dept. of Physics, 1990.

2. W.G.D. Dharmaratna and G.R. Goldstein, Tufts preprint TUFTS-TH-92-G02, under revision for publication.

3. J. Soffer and N.A. Törnqvist, Phys. Rev. Lett. 68, 907 (1992).

4. B. Lundberg et al., Phys. Rev. D40, 3557 (1989).

5. G.L. Kane, G.A. Ladinsky and C.-P. Yuan, Phys. Rev. D45, 124 (1992).

6. R.H. Dalitz and G.R. Goldstein, Phys. Rev. D45, 1531 (1992).

7. J. Qiu and G. Sterman, Phys. Rev. Lett. 67, 2264 (1991).

8. R.H. Dalitz and G.R. Goldstein, Phys. Lett. B287, 225 (1992).

9. F. Abe et al. (CDF Collaboration), Phys. Rev. Lett. 64, 147 (1990); Phys. Rev. Lett. 68, 447 (1992); Lee Pondrom (CDF Collaboration), "Results from Hadron Colliders", in: Proceedings of the XXVth International Conference on High Energy Physics, Singapore, August 2-8, 1990, (ed. by K.K.Phua and Y.Yamaguchi, World Scientific, Singapore, 1991), vol. I, p. 144; K. Sliwa (CDF Collaboration), in: Proc. 25th Rencontre de Moriond: Z ${ }^{0}$ Physics (LesArcs, 4-11 March 1990), (ed. J.Tran Thanh Van, Editions Frontieres, Gif-sur-Yvette, 1990), p. 459.

10. R.H. Dalitz and Gary R. Goldstein, "Where is Top?", based on lectures given at the Erice School (1992); manuscript in preparation.

11. Gary R. Goldstein, K. Sliwa and R.H. Dalitz, Phys. Rev. D47, 957 (1993).

12. Gary R. Goldstein, K. Sliwa and R.H. Dalitz, "A Technique for Observing the Top Quark and Measuring Its Mass at the Tevatron", in: Proc. of XXVIth Intl. Conf. on High Energy Physics, Dallas, TX, Aug. 1992, to be published.

13. L. Gamberg, Ph.D. Dissertation, Tufts Unviersity, in preparation.

14. L. Fadeev and V. Popov, Phys. Lett. B25,29 (1967).

15. L. Gamberg and G. R. Goldstein, "Quantum Mass Corrections in the Skyrme Model", in preparation (1993).

16. E. Witten, Nucl. Phys. B160, 57 (1979); T.H.R. Skyrme, Proc. Roy. Soc. A260, 127 (1961); G.S. Adkins, C. Nappi, E. Witten, Nucl. Phys. B228, 552 (1983); for a review see: I. Zahed and G.E. Brown, Phys. Rep. 142.1 (1986).

17. A. Hayashi, G. Eckart, G. Holzwarth, H. Walliser, Phys. Lett. B 147, 5 (1984); M. Mattis, and M. Karliner, Phys. Rev. D31, 2833 (1985); Phys. Rev. D32, 58 (1985).

18. B. Sakita, Phys. Rev. D12, 1038 (1975); E. Tomboulis, Phys. Rev. D12, 1678 (1975); S. Saito, T. Otofuji, and M. Yasuno, Progr. Theor. Phys. 75, 68 (1986); J. Parmentola, I. Zahed, in: Proceedings of 1987 International Workshop on Low Energy Effective Theory of QCD, (World Scientific 1987), p. 106; K. Ohta, Phys. Lett. B 242, 334 (1990); 259, 404 (1991); Phys. Rev. D43, 2635 (1991); H. Verschelde and H. Verbeke, Nuci. Phys. A495, 523 (1989); M. Uehara, Saga University preprint, SAGA-HE-27 (1989).

19. L. Gamberg and G.R. Goldstein, "Quantized Breather Mode Transition Amplitudes in the 1+1 Dimensional Soliton Theory", in preparation (1993). 
20. G. Holzwarth, Phys. Lett. B 241, 165 (1990); G. Holzwarth, G. Pari, and B. Jennings, Nucl. Phys. A515, 665 (1990).

21. B. Moussallam, Phys. Lett. B272, 196 (1991).

22. S. Coleman and E. Weinberg, Phys. Rev. D7, 1888 (1973).

23. G. Holzwarth, Phys. Lett. B291, 218 (1992).

24. B. Moussallam, Insitut de Physique Nucleaire Universite Paris- Sud, Orsay preprint (1992), and Phys. Lett. B272, 196 (1991). 


\section{FACILITIES}

\section{Computation and networking}

Three upgrades in our computing system occurred during 1993: (1) a DEC VAXstation 3100 was upgraded to a VAXstation $4000 / 90,(2)$ three large $(2.6 \mathrm{~Gb})$ disks were purchased, and (3) two 19-inch high-resolution color monitors were purchased.

The new VAXstation 4000/90 provides about 35 MIPS (million instructions per second) of computing power. Two VAXstation 4000/60 workstations obtained in 1992 each provide 12 MIPS compared with only about 4.5 MIPS for our remaining VAXstation $3100 / 38$. This cluster of 4 VAXstations, providing a total of about 64 MIPS, is presently being heavily used by the CDF and Soudan II experiments. The operating system on these machines is VMS 5.5-2 with DECwindows and the Motif window manager. Both CDF and Soudan II are making considerable use of 2-D and 3-D event displays and analysis programs wh: ih can be viewed on two 19-inch color monitors in the VAXcluster and on a 19-inch HDS color X-terminal. An SGI Indigo workstation also provides color graphics and a 24 MIPS R3000 processor, with software that is compatible with our two aging SGI $4 \mathrm{D} / 25$ servers, all heavily utilized by the Fermilab fixed target experiments E769 and E791. A combined total of about 56 MIPS of UNIX computing is available.

Our MicroVAX II system, which was purchased in 1986, is still working, although the CPU board failed and had to be replaced last year. It provides networking and printing services to both the VMS and UNIX clusters described above. We now have three laser printers, a new HP LaserJet 4, an HP Laserjet+ and a QMS PS-810 with PostScript emulation. The second PostScript printer was sorely needed, since some graphics files would not print on the QMS. A third MicroVAX II, donated to our group by Textron Corporation, is also in use and clustered with the two other MicroVAX II's. These machines are also used by members of the Department of Physics and Astronomy at Tufts for electronic mail, editing, and word processing.

The leased-ine connection to Harvard was terminated when their High Energy Group decided to retire their VAX 8850 system. This left no free port which supported synchronous communication between Harvard and Tufts. We were fortunately able to obtain a leased line to M.I.T. and the use of two $19.2 \mathrm{~Kb}$ modems, increasing the speed of our DECnet link and providing a more direct connection through M.I.T. to Fermilab and to other high energy physics laboratories and universities. This connection provides VMS command and file transfer features which are not available via TELNET and FTP.

As reported last year, all of our computers are linked by ethernet, which connects to the campus-wide network. TGV Multinet software runs on the VMS systems to provide TELNET, FTP, and Internet connections, which are standard features of UNIX systems. We are connected to NEARnet through a $56 \mathrm{~Kb}$ link maintained by Tufts' Computing and Communications Services. It is hoped that the link may soon be upgraded to fractional T1 speed to accommodate the increasing traffic. This connection has proven to be highly reliable, and faster than DECnet for large file transfers.

We have experienced several failures of VT-220 compatible graphics terminals, and we continue to search for reliable replacements. The increasing use of graphics to display 
large detectors and to analyze complex high-multiplicity events gives us incentive to search for low-cost, large-screen displays such as the HDS X-terminal mentioned above. These Xterminals also have much greater graphics capability than conventional terminals because they operate at Ethernet speeds - in our experience, about $256 \mathrm{~Kb}$ over a twisted-pair Ethernet cable as compared to $19.2 \mathrm{~Kb}$ for a conventional terminal.

The Silicon Graphics IRIS 3130 workstation, which we received as a donation last year from Textron Defense Systems, was restored to operational condition by the purchase of a small system disk $(170 \mathrm{Mb})$, since the original was destroyed because of security requirements. We hope to make use of the IRIS's excellent color graphics capabilities for $\mathrm{X}$-windows applications, via its ethernet connection; however, the operating system is an early version of UNIX which is not readily compatible with X-window software, and this monitor is not compatible with most of our other equipment. 


\section{Communications 1992 - 1993 : Experimental}

\section{A.1. Publications}

$\underline{\mathrm{CDF}}$

Search for $\Lambda_{b} \rightarrow J / \psi \Lambda^{\circ}$ in $p \bar{p}$ collisions at $\sqrt{s}=1.8 \mathrm{TeV}$. D. Benjamin, M. Roach-Bellino, K. Sliwa and M. Timko with authors from CDF Collaboration. F. Abe et al., Phys. Rev. D47 Rapid Communications, 2639 (1993).

Measurement of the cross section for production of two isolated prompt photons in $p \bar{p}$ collisions at $\sqrt{s}=1.8 \mathrm{TeV}$. D. Benjamin, M. Roach-Bellino, K. Sliwa and M. Timko with authors from CDF Collaboration, Phys. Rev. Lett. 70, 2232 (1993).

Inclusive $J / \psi, \psi(2 S)$ and $b$-quark production in $p \bar{p}$ collisions at $\sqrt{s}=1.8 \mathrm{Te} \mathrm{V}$. D. Benjamin, M. Roach-Bellino, K. Sliwa and M. Timko with authors from CDF Collaboration, Phys. Rev. Lett. 69, 3904 (1992).

Search for Squarks and Gluinos in $p \bar{p}$ collisions at $\sqrt{s}=1.8 \mathrm{TeV}$. D. Benjamin, M. RoachBellino, K. Sliwa and M. Timko with authors from CDF Collaboration, Phys. Rev. Lett.69, 3439 (1992).

The Dijet Angular Distribution at $\sqrt{s}=1.8 \mathrm{TeV}$. D. Benjamin, M. Roach-Bellino, K. Sliwa and M. Timko with authors from CDF Collaboration, Phys. Rev. Lett. 69, 2897 (1992).

Limits on the production of massive stable charged particles. D. Benjamin, M. RoachBellino, K. Sliwa and M. Timko with authors from CDF Collaboration, Phys. Rev. D46, Rapid Communications, 1889 (1992).

On observing top quark production at the Tevatron. Gary R. Goldstein, K. Sliwa and R.H. Dalitz, Phys. Rev. D47, 967 (1993).

Comparison of jet production in in $p \bar{p}$ collisions at $\sqrt{s}=546$ and $1800 \mathrm{GeV}$. D. Benjamin, M. Roach-Bellino, K. Sliwa and M. Timko with authors from CDF Collaboration, Phys. Rev. Lett. 70, 1376 (1993).

A measurement of jet shapes in p $\bar{p}$ collisions at $\sqrt{s}=1.8 \mathrm{TeV}$. D. Benjamin, M. RoachBellino, K. Sliwa and M. Timko with authors from CDF Collaboration, Phys. Rev. Lett. 70, 713 (1993).

Limits on the rare decay $W^{ \pm} \rightarrow \gamma \pi^{ \pm}$in $p \bar{p}$ collisions at $\sqrt{s}=1.8 \mathrm{TeV}$. D. Benjamin, M. Roach-Bellino, K. Sliwa and M. Timko with authors from CDF Collaboration, Phys. Rev. Lett. 69, 2160 (1992).

$A$ measurement of the production and muonic decay rate of $W$ and $Z$ bosons in $p \bar{p}$ collisions at $\sqrt{s}=1.8$ TeV. D. Benjamin, M. Roach-Bellino, K. Sliwa and M. Timko with authors from CDF Collaboration, Phys. Rev. Lett. 69, 28 (1992).

Fermilab E - 632

Coherent production of single pions and $\rho$ mesons in charged-current interactions of neutrinos and antineutrinos on neon nuclei at the Fermilab Tevatron. S. Willocq, H. Akbari, T. Kafka, R.H. Milburn, A. Napier, D. Passmore, and J. Schneps, with authors from the E632 Collaboration, Phys. Rev. D47, 2661 (1993).

Fermilab E - 691

Study of the decays $D \rightarrow K 3 \pi$. K. Sliwa with authors from the Tagged Photon Collaboration, J.C. Anjos et al., Phys. Rev. D46, 1941 (1992).

Experimental probes of final state interactions in $D^{\circ}$ meson decays. K. Sliwa with authors from the Tagged Photon Collaboration, Phys. Rev. D46, 1 (1992).

Study of the decays $D \rightarrow K \pi \pi \nu$ and $D \rightarrow K^{*} \pi \pi e \nu$. K. Sliwa with authors from the Tagged Photon Collaboration, Phys.Rev. D45, 2177 (1992). 
Fermilab E - 769

Feynman-x and transverse momentum dependence of $D^{ \pm}$and $D^{0}, \bar{D}^{0}$ production in 250 GeV $\pi^{-}$-nucleon interactions. J. Metheny, R.H. Milburn, and A. Napier, with authors from the E769 Collaboration, Phys. Rev. Lett. 69, 3147 (1992).

Atomic mass dependence of $D^{ \pm}$and $D^{0}, \bar{D}^{0}$ production in $250 \mathrm{GeV} \pi^{ \pm}$-nucleon interactions. J. Metheny, R.H. Milburn, and A. Napier, with authors from the E769 Collaboration, Phys. Rev. Lett. 70, 722 (1993).

Soudan - II

The atmospheric flux $\nu_{\mu} / \nu_{e}$ anomaly as manifestation of proton decay $p \rightarrow e^{+} \nu \nu$. W.A. Mann, T. Kafka, and W. Leeson, Phys. Lett. B 291200 (1992).

Search for magnetic monopoles with the Soudan 2 detector. D. Benjamin, B. Ewen, T. Kafka, J. Kochocki, W. Leeson, W.A. Mann, L. McMaster, R.H. Milburn, A. Napier, W. Oliver, B. Saitta, J. Schneps, and N. Sundaralingam, with authors from the Soudan-II collaboration, Phys. Rev. D46 4846 (1992).

\section{A.2. Publications Submitted}

$\underline{\mathrm{CDF}}$

Observation of the decay $B_{s}^{\circ} \rightarrow J / \psi \phi$ in $p \bar{p}$ collisions at $\sqrt{s}=1.8 \mathrm{TeV}$. D. Benjalain, M. Roach-Bellino, K. Sliwa and M. Timko with authors from CDF Collaboration, Preprint FERMILAB-PUB-93/141-E, submitted to Phys. Rev. Lett., June 1993.

Measurement of $B$ meson and $b$ quark cross-section at $\sqrt{s}=1.8 \mathrm{TeV}$ using the exclusive de cay $B^{\circ} \rightarrow J / \psi K^{*}(892)^{\circ}$. D. Benjamin, M. Roach-Bellino, K. Sliwa and M. Timko with authors from CDF Collaboration, Preprint FERMILAB-PUB-93/131-E, submitted to Phys. Rev. Lett., June 1993.

Measurement of the bottom quark production cross-section in $p \bar{p}$ collisions at $\sqrt{s}=1.8 \mathrm{TeV}$ using semileptonic decay to muons. D. Benjamin, M. Roach-Bellino, K. Sliwa and M. Timko with authors from CDF Collaboration, Preprint FERMILAB-PUB-93/145-E, submitted to Phys. Rev. Lett., June 1993.

Inclusive $\chi_{c}$ and $b$ quark production in pp collisions at $\sqrt{s}=1.8 \mathrm{TeV}$. D. Benjamin, M. Roach-Bellino, K. Sliwa and M. Timko with authors from CDF Collaboration, Preprint FERMILAB-PUB-93/106-E, submitted to Phys. Rev. Lett., May 1993.

Measurement of the bottom quark production cross-section in $p \bar{p}$ collisions at $\sqrt{s}=1.8 \mathrm{TeV}$ using semileptonic decay to electrons. D. Benjamin, M. Roach-Bellino, K. Sliwa and M. Timko with authors from CDF Collaboration, Preprint FERMILAB-PUB-93/091-E, submitted to Phys. Rev. Lett., June 1993.

A search for First-Generation Leptoquarks in $p \bar{p}$ collisions at $\sqrt{s}=1.8$ TeV at CDF. D. Benjamin, M. Roach-Bellino, K. Sliwa and M. Timko with authors from CDF Collaboration, Preprint FERMILAB-PUB-93/070-E, submitted to Phys. Rev. D, Rapid Communications, April 1993.

Measurement of jet multiplicity in $W$ events produced, in pp collisions at $\sqrt{s}=1.8 \mathrm{TeV}$. D. Benjamin, M. Roach-Bellino, K. Sliwa and M. Timko w th authors from CDF Collaboration, Preprint FERMILAB-PUB-93/063-E, submitted to Phys. Sev. Lett., March 1993.

The center-of-mass angular distribution of prompt photons produced in $p \bar{p}$ collisions at $\sqrt{s}=1.8 \mathrm{TeV}$. D. Benjamin, M. Roach-Bellino, K. Sliwa and M. Tirkko with authors from CDF Collaboration, Preprint FERMILAB-FUB-93/032-E, submitted to Phys. Rev. Lett., March 1993.

Measurement of the dijet mass distribution in $p \bar{p}$ collisions at $\sqrt{s}=1.8 \mathrm{TeV}$. D. Benjamin, M. Roach-Bellino, K. Sliwa and M. Timko with authors from CDF Collaboration, Preprint FERMILAB-PUB-93/017-E, submitted to Phys. Rev. Lett., February 1993. 
A study of four-jet events and evidence for double parton interactions in p $\bar{p}$ collisions at $\sqrt{s}=1.8 \mathrm{Te} V$. D. Benjamin, M. Roach-Bellino, K. Sliwa and M. Timko with authors from CDF Collaboration, Preprint FERMILAB-PUB-93/003-E, submitted to Phys. Rev. Lett., January 1993.

Fermilab E - 691

A Dalitz plot analysis of $D \rightarrow K \pi \pi$ decays. K. Sliwa with authors from the Tagged Photon Collaboration, Preprint FERMILAB-PUB-92/284-E, submitted to Phys. Rev. Lett.

Fermilab E - 769

$D^{* \pm}$ production in $250 \mathrm{GeV} \pi^{ \pm} N$ interactions. J. Astorga, J. Metheny, R.H. Milburn, A. Napier, and D. Passmore, with authors from the E769 Collaboration, submitted to Phys. Rev. Lett.

\section{A.3. Work in Preparation}

Fermilab E-632

Neutral strange particle production in neutrino and antineutrino charged-current interactions on neon. H. Akbari, T. Kafka, R.H. Milburn, A. Napier, D. Passmore, J. Schneps, and S. Willocq with authors from the E632 Collaboration. To be submitted to Phys. Rev. D.

Fermilab E - 769

Absolute cross sections and incident particle dependence of charm meson hadroproduction at $250 \mathrm{GeV}$. J. Astorga, J. Metheny, R.H. Milburn, A. Napier, and D. Passmore, with authors from the E769 Collaboration, to be submitted to Phys. Rev. Lett.

Soudan - II

Tracking calorimeter modules for the Soudan 2 detector. D. Benjamin, B. Ewen, T. Kafka, J. Kochocki, W. Leeson, W.A. Mann, L. McMaster, R.H. Milburn, A. Napier, W. Oliver, B. Saitta, J. Schneps, and N. Sundaralingam, to be submitted to Nuclear Instruments and Methods.

Observation of a possible excess of underground muons from the vicinity of 3 C279. B. Ewen, T. Kafka, J. Kochocki, W. Leeson, W.A. Mann, R.H. Milburn, A. Napier, W. Oliver, B. Saitta, J. Schneps, and N. Sundaralingam, to be submitted to Phys. Lett. B.

\section{A.4. Published Conference Papers}

\section{$\underline{\mathrm{CDF}}$}

Measurement of $\sigma \cdot B R(W+\gamma)$ and $\sigma \cdot B R(Z+\gamma)$ at $C D F$. D. Benjamin, invited talk at XXVII Rencontres de Moric d, Les Arcs, France, March 1993. To be published in the Proceedings.

Fermilab E-632

Neutral secondary vertices associated to high energy neutrino interactions. Proceedings of the Neutrino'92 International Conference on Neutrino Physics and Astrophysics, Nucl. Phys. B (Proc. Suppl.) 31, 284 (1993).

Strangeness production in neutrino interactions. Proceedings of the XXVI Int. Conference on High Energy Physics, Dallas, TX, Aug. 1992. American Institute of Physics, New York 1993, Vol. I, p. 802.

Neutral strange-particle production in neutrino-neon charged-current interactions at the Fermilab Tevatron. Proceedings of the DPF92 Meeting (Division of Particles and Fields of the American Physical Society), Fermilab, Nov. 1992. World Scientific, Singapore 1993, p. 731. 


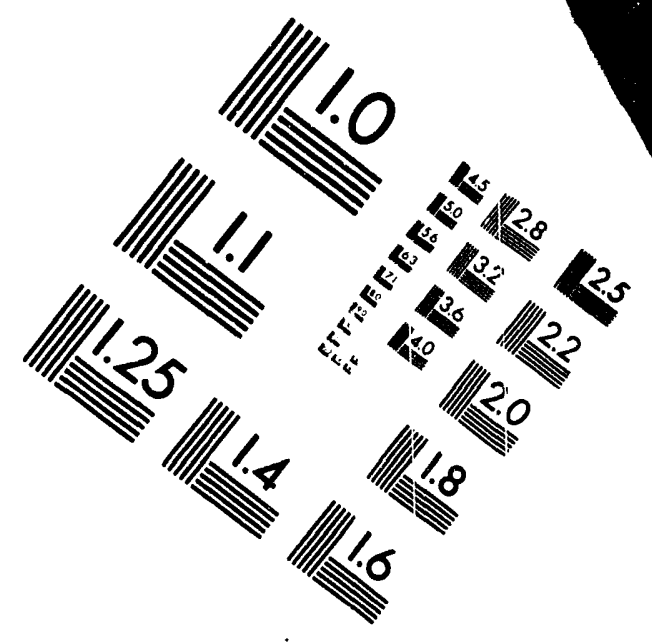

\section{Centimeter}

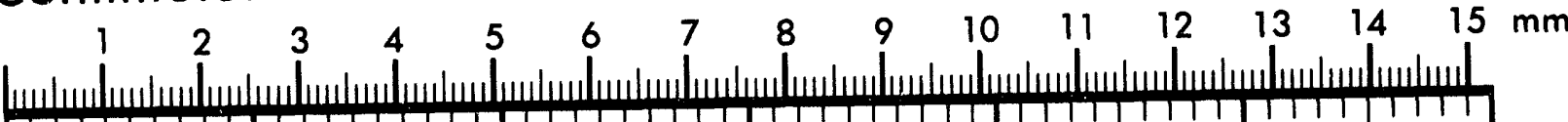

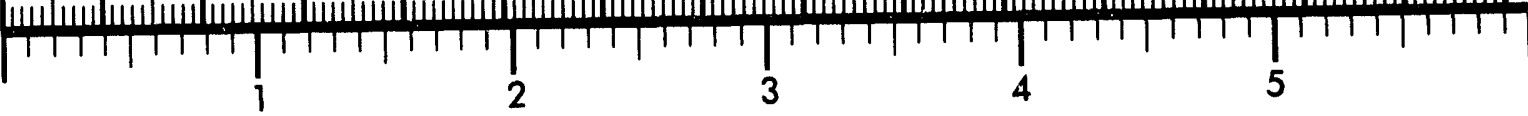
Inches
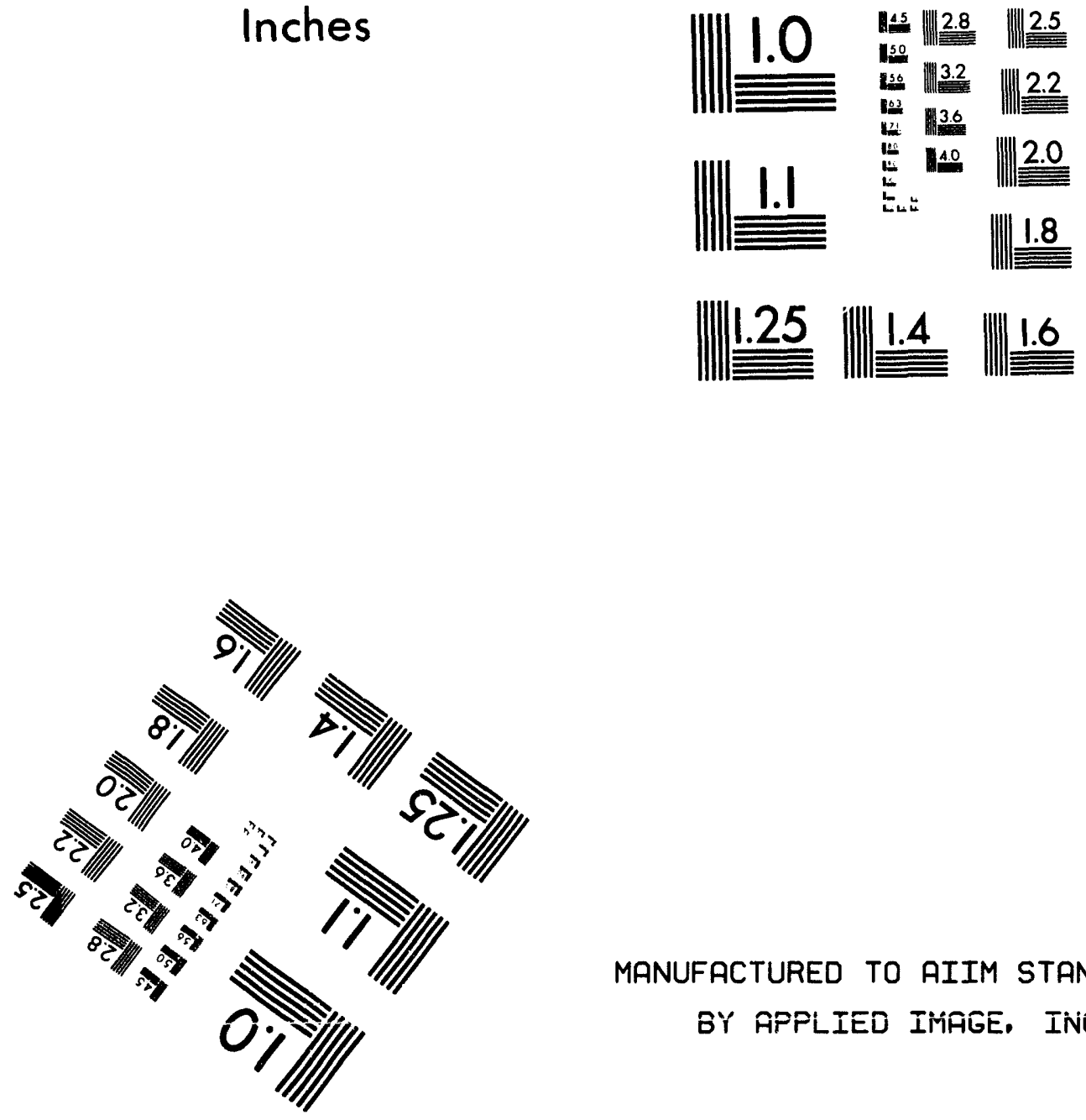

MANUFACTURED TO AIIM STANDARDS BY APPLIED ITHÁGE. INC.

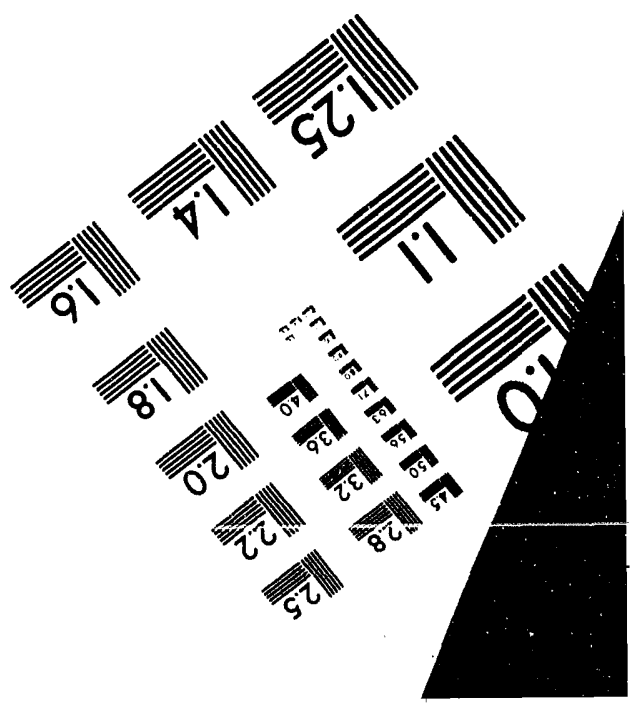



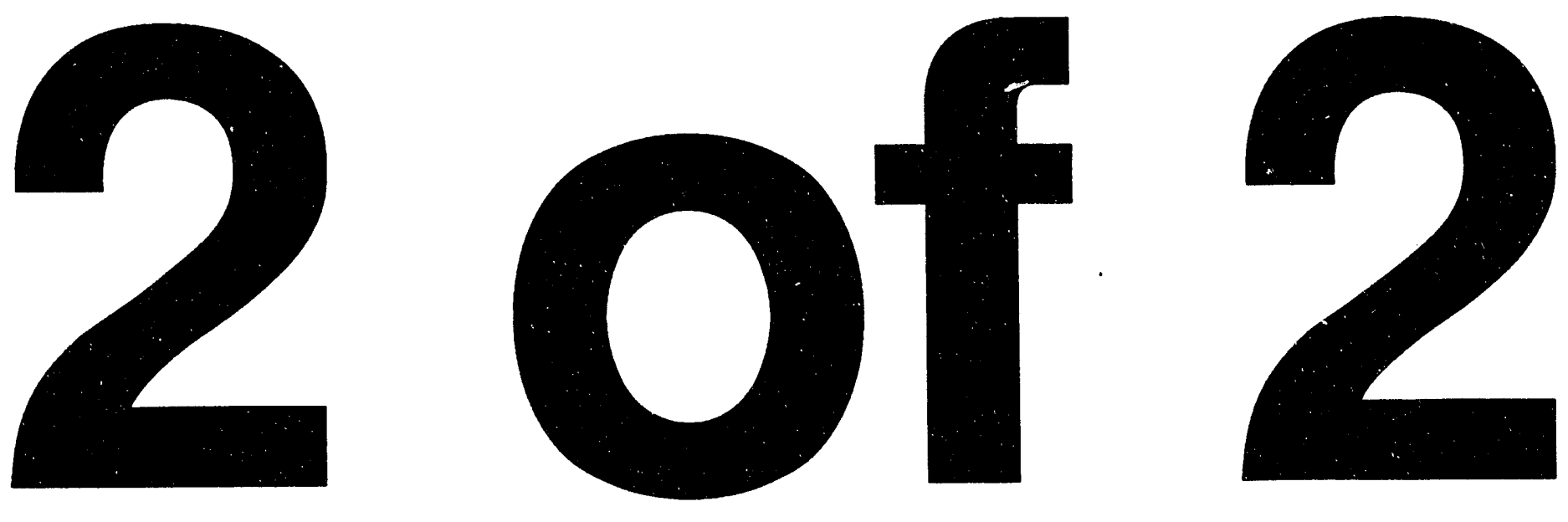
$\underline{\text { Fermilab E - } 769 \text { and E - } 791}$

Fermilab E791. Proceedings of the XXVI Int. Conference on High Energy Physics, Dallas, TX, Aug. 1992, publ. by The American Institute of Physics, New York 1993, Vol. I, p. 1058.

Feynman-x arud transverse momentum dependence of $D^{ \pm}, D^{0}, \bar{D}^{0}$, and $D^{* \pm}$ production in $250 \mathrm{GeV} \pi$-nucleon interactions. E769 Collaboration, Proceedings of the XXVI Int. Conference on High Energy Physics, Dallas, TX, Aug. 1992, publ. by The American Institute of Physics, New York 1993, Vol. I, p. 1054.

E791 Status Report. Proceedings of the DPF92 Meeting (Division of Particles and Fields of the American Physical Society), Fermilab, Nov. 1992. World Scientific, Singapore 1993, Vol. I, p. 672.

Atomic mass dependence of $D^{ \pm}$and $D^{0}, \bar{D}^{0}$ production in $250 \mathrm{GeV} \pi^{ \pm}$-nucleon interactions. E769 Collaboration, Proceedings of the DPF92. Meeting (Division of Particles and Fields of the American Physical Society), Fermilab, Nov. 1992. World Scientific, Singapore 1993, Vol. I, p. 718.

Feynman-x and transverse momentum dependence of $D^{ \pm}, D^{0}, \bar{D}^{0}$, and $D^{* \pm}$ production in $250 \mathrm{GeV} \pi$-nucleon interactions. E769 Collaboration, Proceedings of the DPF92 Meeting (Division of Particles and Fields of the American Physical Society), Fermilab, Nov. 1992. World Scientific, Singapore 1993, Vol. I, p. 721.

$A$ study of beauty and charm production in $250 \mathrm{GeV} \pi^{+}$-nucleon interactions using semielectronic decays. E769 Collaboration, Proceedings of the DPF92 Meeting (Division of Particles and Fields of the American Physical Society), Fermilab, Nov. 1992. World Scientific, Singapore 1993, Vol. I, p. 725.

\section{$\underline{\text { Soudan - II }}$}

The Soudan-2 experiment. T. Kafka, for the Soudan-2 collaboration, Nucl. Phys. B (Proc. Suppl.) 28A, 377-381 (1992).

Search for $A G N$ neutrinos with the Soudan 2 detectcr. Proceedings of the Workshop on High Energy Astrophysics, Honolulu, Hawaii, April 1992.

Results from the Soudan 2 detector. Proceedings of the XXVI Int. Conf. on High Energy Physics, Dallas, Texas, August 1992, publ. by The American Institute of Physics, New York 1993, Vol. I, p. 1232.

Underground muons from point sources. Proceedings of the XXVI Int. Conf. on High Energy Physics, Dallas, Texas, August 1992, publ. by The American Institute of Physics, New York 1993, Vol. I, p. 1238.

Anomalously low atmospheric $\nu_{\mu} / \nu_{e}$ ratio as manifestation of $p \rightarrow e^{+} \nu \nu$. W.A. Mann, T. Kafka, and W. Leeson, Proceedings of DPF'92, Meeting of the Division of Particles and Fields of the American Physical Society, Fermilab, 10-14 November 1992, ed. C.H. Albright, P.H. Kasper, R. Raja, and J. Yoh, World Scientific, Singapore 1993, Vol. II, p. 1330 .

Fermilab $\mathrm{P}-803, \mathrm{P}-822$

Neutrino oscillations: past, present, and future. J. Schneps, Nucl. Phys. B (Proc. Suppl.) 31, 307-318 (1993). 


\section{A.5. Conference Presentations}

Soudan - II

Status of the Soudan-II proton stability experiment. W.A. Mann, talk presented at the International Workshop on Supersymmetry and Unification of Fundamental Interactions SUSY 93, Northeastern University, Boston MA, March 31, 1993.

Atmospheric neutrino anomaly as proton decay. W.A. Mann, talk presented at the Atmospheric Neutrino Workshop, LSU-HEPA-93-6, Louisiana State University, May 6-8, 1993.

\section{A.6. Papers submitted to conferences}

Soudan - II

Status of the Soudan 2 detector experiment. D. Benjamin, B. Ewen,T. Kafka, J. Kochocki, W. Leeson, W.A.Mann, L. McMaster, R. Milburn, A. Napier, W. Oliver, J. Schneps, N. Sundaralingam, with authors from the Soudan-II collaboration, submitted to the Proceedings of the 23rd International Cosmic Ray Conference, Calgary, July 1993.

Contained $\nu$ events observed in Soudan 2. Tufts authors, with authors from the Soudan-II collaboration, submitted to the Proceedings of the 23rd International Cosmic Ray Conference, Calgary, July 1993.

Ultra high energy cosmic ray composition from simultaneous surface and underground measurements at Soudan 2. Tufts authors, with authors from the Soudan-II collaboration, submitted to the Proceedings of the 23rd International Cosmic Ray Conference, Calgary, July 1993.

12.A.7. Ph. D. Theses

Soudan - II

Investigation of the relative abundance of heavy versus light nuclei in primary cosmic rays using underground muon bundles. Nakamuthu Sundaralingam, June 1993. (Issued as Report DOE/ER/40702-3. )

\section{A.8 Technical Notes}

\section{$\underline{\mathrm{CDF}}$}

Measurement of $\sigma \cdot B R(W+\gamma)$ and $\sigma \cdot B R(Z+\gamma)$ in the electron and muon channels in $\sqrt{s}=$ $1.8 \mathrm{Te} V p \bar{p}$ collisions. Note CDF/ANAL/ELECTROWEAK/CDFR/1941. D. Benjamin, M. Roach, K. Sliwa and M. Timko, with 9 authors from CDF Collaboration.

Search for $t \bar{t}$ events in the lepton and four jets final state. Note CDF/TOP/CDFR/1993. K. Sliwa.

Sample selection for the lepton+jets channel top search.

Note CDF/ANAL/TOP/CDFR/2065. K. Sliwa with 29 authors from CDF Collaboration.

$\underline{\text { Soudan - II }}$

Users guide to the TASSO array. Soudan-II note PDK-524, K. Karr, M. Pfenning, W.A. Mann, W. Oliver, Y. Gopika, T. Kafka, with authors from Oxford Univ., Sept. 1992.

The HPW enhancement of the active shield. Soudan-II note PDK-525, B. Ewen, W.A. Mann, W.P. Oliver, N. Sundaralingam, Sept. 1992.

Nucleon decay interpretation of the atmospheric flux $\nu_{\mu} / \nu_{e}$ anomaly within $S O(10)$ grand unification. Soudan-II note PDK-538, Allen E. Everett, W. Anthony Mann, Stephane Willocq, December 1992.

A Frejus view of the proton decay scenario. Soudan-II note PDK-542, W.A. Mann, December 1992. 


\section{Solenoidal Detector Collaboration}

Electro - mechanics of drift tube wires. SDC note SDC-92-320, R.H. Milburn, Sept. 1992. SDC 9-cm muon drift tube: Electromechanical studies - I. SDC note SDC-92-321, G. Yasuda and R.H. Milburn, Sept. 1992.

Ovality tolerances and muon tube precision. SDC note SDC-93-519, R.H. Milburn, May 1993.

\section{A.9. Media Communications}

Soudan - II

Could protons be mortal after all? SCIENCE, Vol. 257, Sept. 25, 1992, pp. 1862-1863; W.A. Mann interviewed; reported by Faye Flam.

Proton decay theory may be in for revival from new approach. The New York Times, Sept. 29, 1992; p. C7; W.A. Mann interviewed; reported by Malcolm W. Browne.

The search for proton decay. BBC World Service, Discovery Edition, November 24 and 25, 1992; interview with W.A. Mann, producer/commentator Julian Brown.

The trouble with atmospheric neutrinos. New Scientist, March 13, 1993, p. 16; summary of work by W.A. Mann, T. Kafka, and W. Leeson, reported by Christine Sutton.

New Soudan detector nears completion. CERN Courier, Vol. 33, pp. 16-21, April 1993.

\section{A.10. Invited Seminars}

Soudan - II

The atmospheric flux $\nu_{\mu} / \nu_{e}$ anomaly as manifestation of proton decay $p \rightarrow e^{+} \nu \nu$. W.A. Mann, Joint CFA-Tufts Cosmology Seminar, Nov. 17, 1992.

The low atmospheric neutrino flavor ratio - a manifestation of proton decay? W.A. Mann, Harvard University, Dec. 2, 1992.

Does the proton decay? W.A. Mann, Department of Energy Headquarters in Germantown, Maryland, January 28, 1993.

Do neutrinos oscillate? Does the proton decay? W.A. Mann, Brandeis University, April 27, 1993.

\section{A.11. Proposals}

Boston Area Center for Development of an SDC Precision Muon System (Grant renewal). T. Kafka, W.A. Mann, R.H. Milburn, A. Napier, K. Sliwa, with authors from Brandeis and Harvard Universities, submitted to the Texas National Research Laboratory Commission, February 1993. 


\section{Communications $1992-1993$ : Theoretical}

\section{B.1. Publications}

The Analysis of Top-Antitop Production and Dilepton Decay Events and the Top Quark Mass. R.H. Dalitz and G.R. Goldstein, Phys. Lett. B287, 225 (1992).

On Observing Top Quark Production at the Tevatron. G.R. Goldstein, K. Sliwa and R.H. Dalitz, Phys. Rev. D47, 957 (1993).

A Technique for Observing the Top Quark and Measuring Its Mass at the Tevatron. G.R. Goldstein, K. Sliwa and R.H. Dalitz, Proceedings of the XXVI International Conference on High Energy Physics, Dallas, TX, Aug. 6-12, 1992, ed. by James R. Sanford, publ. by the American Institute of Physics, New York 1993, Vol. I, p. 1027.

\section{B.2. Publications Submitted}

Single Particle Polarization in QCD Subprocesses. (Tufts preprint TUFTS-TH-92-G02.) W.G.D. Dharmaratna and G.R. Goldstein, being revised for publication, 1993.

\section{B.3. Work in Preparation}

Where is Top? (Lectures given at Erice Summer School, 1992.) R.H. Dalitz and G.R. Goldstein, in preparation for publication in Proceedings.

Photoproduction and Polarization Experiments. F. Arash, M.J. Moravcsik, and G.R. Goldstein, being revised for publication (1993).

Polarized Electrons are Unnecessary to Determine Elastic Form-factors. G.R. Goldstein and M.J. Moravcsik, under revision for publication (1993).

Amplitude Phase Patterns: a New Look at Strong Interactions. G.R. Goldstein, F. Arash, and M.J. Moravcsik, being revised for publication (1993).

\section{B.4. Papers submitted to conferences}

An Improved Kinematic Method for Observing the Top Quark in $p \bar{p}$ Collisions. G.R. Goldstein and R.H. Dalitz, contributed to the XVI International Symposium on Lepton-Photon Interactions, Cornell University, Aug. 1993. 

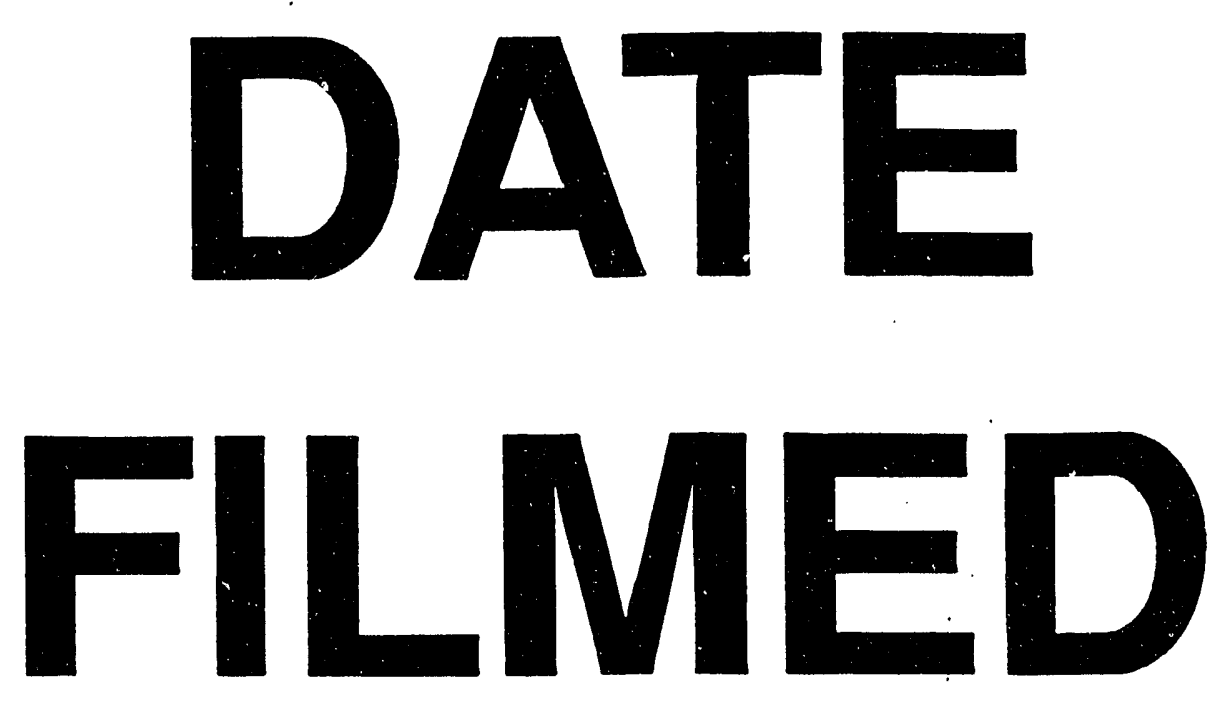

$9 / 28 / 93$

